\title{
An Exploration of Intolerance of Uncertainty and Memory Bias
}

\author{
Kylie Francis
}

A Thesis

In the Department of Psychology

Presented in Partial Fulfillment of the Requirements

For the Degree Doctor of Philosophy at

Concordia University

Montréal, Québec,

Canada

(C) Kylie Francis, 2011 


\section{CONCORDIA UNIVERSITY \\ SCHOOL OF GRADUATE STUDIES}

This is to certify that the thesis prepared

By: $\quad$ Kylie Francis

Entitled: $\quad$ An Exploration of Intolerance of Uncertainty and Memory Bias

and submitted in partial fulfillment of the requirements for the degree of

Doctor of Philosophy (Clinical Psychology)

complies with the regulations of the University and meets the accepted standards with respect to originality and quality.

Signed by the final examining committee:

Dr. L. Kalman (Physics) $\quad$ Chair

Dr. Meredith Coles (Psychology) External Examiner

Dr. H. Osana (Education) External to Program

Dr. A. Radomsky (Psychology) Examiner

Dr. N. Phillips (Psychology) $\quad$ Examiner

Dr. M. Dugas (Psychology) Thesis Supervisor

Approved by

Dr. Andrew Chapman (Ph.D. Graduate Program Director)

Chair of Department or Graduate Program Director

Dr. Brian Lewis (Dean and Chair, Faculty of Arts and Science)

Dean of Faculty 


\begin{abstract}
An Exploration of Intolerance of Uncertainty and Memory Bias

Kylie Francis, Ph.D.

Concordia University, 2011
\end{abstract}

Research shows that Intolerance of Uncertainty (IU) is related to excessive worry. Individuals high in IU appear to have information processing biases, which may explain how IU leads to worry. Specifically, high IU individuals appear to have an attentional bias for threat and uncertainty, and interpret uncertain information in a threatening way. While there is some evidence of a memory bias for uncertainty in high IU, findings are limited. The purpose of this study was therefore to explore the relationship between IU and memory biases for threat and uncertainty. Stimuli for use in the research were pilot tested in Study 1; this produced a set of word stimuli that included neutral, uncertain, social threat, physical threat, and positive words. Study 2 evaluated the relationship between IU and explicit memory for threat and uncertainty; the influence of worry, cognitive avoidance, and depression were also explored. Participants performed an incidental learning task and then completed recognition and free recall tests of memory. Results showed no relationship between IU and explicit memory for uncertain and threat words. However, three forms of cognitive avoidance-distraction, avoidance of threatening stimuli, and thought suppression-were related to recall of physical threat words. Study 3 examined the role of IU in implicit memory for threat and uncertainty, and the contribution of cognitive avoidance and stimulus relevance. Participants performed an implicit encoding task, and measures of explicit (recall) and implicit (tachistoscopic identification) memory. The results showed no relationship between IU and memory for threat or uncertainty. However, stimulus relevance was related to implicit memory for physical threat words. Interesting results again emerged for cognitive avoidance: thought substitution, avoidance of threatening stimuli, and thought suppression were related to implicit memory for physical threat words. Findings from the three studies were discussed in relation to the Williams et al. (1997) model, which predicts an implicit but not an explicit memory bias for threat among anxious individuals. Further exploration of stimulus relevance and cognitive avoidance were recommended for future research. 


\section{Acknowledgements}

First and foremost, I would like to thank my thesis advisor, Dr. Michel J. Dugas. A surprising number of years ago, Michel decided to take me under his wing. He provided invaluable career advice, and then supported me at every stage of my development as a psychologist. Michel, I couldn't have done it without you. Thank you. Thanks are also due to the wonderful group of volunteers, research assistants, undergraduate students, and graduate students of the Dugas lab, who were so generous with their time. Special thanks go to Dr. Naomi Koerner and Nicole Heineke, who helped with Study 1. Research assistants Mary Hedayati, Kristin Anderson, and Tyler Tulloch were capable and cheerful in the face of even the most unreasonable requests. Thanks also go to Anthony Hopley, whose undergraduate research on stimulus relevance informed my own. To Dr. Nina Laugesen: thank you for your help, your wisdom, and your friendship - and for "going first". To Steve: thank you for being my rock, my stick, and my carrot. Finally, I dedicate this thesis to my parents, Ken and June Francis, who taught me to dream, and then showed me that dreams are built on hard work and determination. Your love and support made everything possible.

This research was supported by a grant from the Fonds de la Recherche en Santé Québec. 


\section{Table of Contents}

List of Figures and Tables............................................. vi

Introduction................................................................ 1

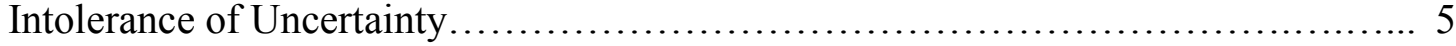

Intolerance of Uncertainty and Information Processing $\ldots \ldots \ldots \ldots \ldots \ldots \ldots \ldots \ldots \ldots$

Information Processing in GAD and Worry.............................. 12

Information Processing in High Trait Anxiety............................... 33

Information Processing in Other Anxiety Disorders............................ 37

Summary of Information Processing Research.............................. 39

Critique of Research on Memory Bias in GAD and Worry $\ldots \ldots \ldots \ldots \ldots \ldots \ldots \ldots . .40$

Theoretical Accounts of Existing Findings................................... 51

General Goals of the Current Research..................................... 59

Study Goals and Hypotheses............................................... 62

Study 1

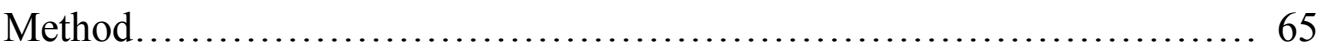

Results.................................................... 71

Discussion....................................................... 79

Study 2

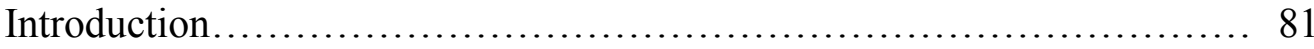

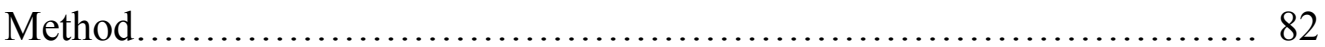

Results........................................................ 90

Discussion................................................ 110

Study 3

Introduction..................................................... 117

Method........................................................ 119

Results...................................................... 132

Discussion..................................................... 156

General Discussion................................................. 161

Suggestions for Future Research....................................... 171

References.......................................................... 177

Appendices........................................................ 205 


\section{List of Figures and Tables}

\section{Figures}

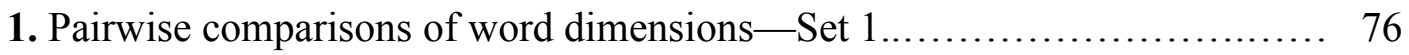

2. Pairwise comparisons of word dimensions - Set $2 \ldots \ldots \ldots \ldots \ldots \ldots \ldots \ldots \ldots \ldots \ldots \ldots \ldots$

3. Study 3 stimuli, Sets A \& B........................................ 123

\section{Tables}

1. Matched stimulus words............................................... 74

2. Study 2 means and standard deviations.............................. 92

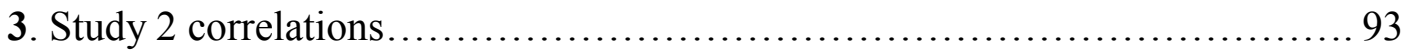

4. Study 2: IU predicting sensitivity for neutral words...................... 98

5. Study 2: IU predicting sensitivity for uncertain words..................... 99

6. Study 2: IU predicting sensitivity for threat words...................... 100

7. Study 2: IU predicting sensitivity for positive words $\ldots \ldots \ldots \ldots \ldots \ldots \ldots \ldots \ldots 1$

8. Study 2: CAQ thought subs predicting recall for threat words............. 105

9. Study 2: CAQ transformation predicting recall for threat words.............. 106

10. Study 2: CAQ distraction predicting recall for threat words............... 107

11. Study 2: CAQ avoidance predicting recall for threat words................. 108

12. Study 2: CAQ thought suppress predicting recall for threat words......... 109

13. Study 3 means and standard deviations............................. 135

14. Study 3 correlations................................................. 136

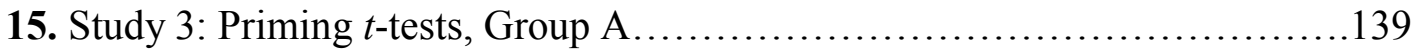

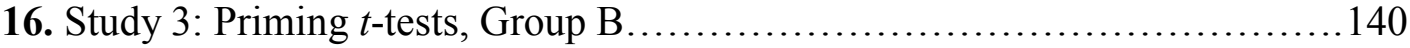

17. Study 3: IU \& SR predicting priming for neutral words................... 144

18. Study 3: IU \& SR predicting priming for uncertain words $\ldots \ldots \ldots \ldots \ldots \ldots \ldots . . \ldots 146$

19. Study 3: IU \& SR predicting priming for threat words.................. 147

20. Study 3: IU \& SR predicting priming for positive words.................. 148

21. Study 3: CAQ thought subs \& SR predicting priming for threat words....... 150

22. Study 3: CAQ transformation \& SR predicting priming for threat words... 151

23. Study 3: CAQ distraction \& SR predicting priming for threat words........ 152

24. Study 3: CAQ avoidance \& SR predicting priming for threat words......... 153

25. Study 3: CAQ thought suppress \& SR predicting priming for threat words... 154 


\section{Introduction}

Worry is a widespread experience. Most of us have worried about an event whose outcome is unknown, and whose consequences could range anywhere from disagreeable to disastrous. Research suggests that as much as $38 \%$ of the general population worry at least once a day (Tallis, Davey, \& Capuzzo, 1994). In the literature, worry is often described as an internal monologue concerning some anxiety-provoking future event. For the purposes of research, worry has more specifically been defined as "a cognitive phenomenon... concerned with future events where there is uncertainty about the outcome, the future being thought about is a negative one, and this is accompanied by feelings of anxiety" (MacLeod, Williams, \& Bekerian, 1991, p. 478). While worry is usually accompanied by the experience of anxiety, there is evidence that worry and anxiety are distinct constructs, with different relationships to indices of affect, performance, coping, and problem solving (i.e., Davey, 1993; Davey, Hampton, Farrell, \& Davidson, 1992; Schwartz, Davidson, \& Goleman, 1978; Steptoe \& Kearsley, 1990; Zebb \& Beck, 1998). At mild levels, worry is not only a common experience, but may even be a constructive one. For example, Borkovec (1985) described worry as a form of attempted mental problem solving, whose goal is to generate ways of coping with potential negative outcomes. Studies show that many people believe worrying is helpful. For example, beliefs that worry is motivating, helps problem solving, analytical thinking, and prepares one for negative outcomes, have been widely reported (i.e., Borkovec \& Roemer, 1995; Davey, Tallis, \& Capuzzo, 1996; Freeston, Rheaume, Letarte, Dugas, \& Ladouceur, 1994; Tallis et al., 1994). There is some research support for the idea that worry is useful. For example, Davey and colleagues (1992) found that when trait anxiety 
is controlled, worrying can be related to constructive forms of coping such as information-seeking, monitoring, and problem solving. However, this type of result has emerged primarily when measures of non-clinical worry are used (e.g., Davey et al., 1992; Davey, 1993, 1994a), suggesting that worry may be related to positive coping strategies primarily among low worriers.

In accordance with this idea, research shows that higher levels of worry are often experienced as aversive, intrusive, and uncontrollable (Borkovec \& Newman, 1999; Borkovec, Robinson, Pruzinsky, \& DePree, 1983; Davey, 1993). Subjective reports suggest that people also believe that worry makes them less effective, creates emotional distress, and makes problems seem worse (Borkovec \& Roemer, 1995; Davey et al., 1996; Tallis et al., 1994). Research does in fact indicate that worry can interfere with functioning in a variety of ways. For example, studies show that worry results in slowed performance on certain tasks, particularly when the correct response is unclear (Dugas, Freeston, Blais, \& Ladouceur, 1993; Metzger, Miller, Cohen, Sofka, \& Borkovec, 1990; Tallis, Eysenck, \& Mathews, 1991). In addition, research suggests that worry, rather than anxious affect, has a negative impact on test performance (Deffenbacher, 1978; Morris \& Liebert, 1970; Seipp, 1991). In addition, high worriers appear to require excessive levels of information in order to make decisions (Tallis et al., 1991). Still other research shows greater degrees of procrastination and perfectionism among high worriers, which seems due to their elevated concern about taking action and making mistakes (Frost, Marten, Lahart, \& Rosenblate, 1990; Stöber \& Joormann, 2001). As previously noted, while worry resembles certain aspects of problem solving (i.e., the identification of problems), research suggests that high worry rarely leads to successful problem resolution. While 
worriers do not appear to be deficient in problem solving skills (Davey, 1994b; Dugas, Letarte, Rhéaume, Freeston, \& Ladouceur, 1995), they do appear to have a negative problem orientation - in other words, worriers report a negative view of problems, and a lack of confidence in their own problem solving abilities (Belzer, D'Zurilla, \& MaydeuOlivares, 2002; Davey, 1994b; Dugas, Freeston, \& Ladouceur, 1997). Research has shown that both problem solving skills and a positive problem orientation are necessary for the successful resolution of problems (D’Zurilla \& Nezu, 1999). Worriers may therefore be less successful problem solvers because their negative problem orientation prevents them from acting on any of the ideas generated during the worry process (Davey, 1994b; Davey et al., 1992; Dugas, Letarte, et al., 1995). It has further been proposed that the vigilant, information-seeking cognitive style of worriers may lead them to view innocuous events as threats, and to detect an ever-increasing number of problems, thereby perpetuating the cycle of worry (Davey et al., 1992). Overall, therefore, it appears that worry, particularly at higher levels, is an intrusive, unwanted process that maintains anxiety and awareness of threat, without leading to successful resolution of the triggering situation.

The type of maladaptive worry described above is particularly prominent in Generalized Anxiety Disorder (GAD); in fact, excessive and uncontrollable worry is the central feature of this disorder. In GAD, excessive worry must be present most days for at least six months, and accompanied by physical symptoms such as agitation, muscle tension, irritability, fatigue, and sleep disturbance (American Psychiatric Association, 2000). As already noted, worry is also present in non-clinical populations. However, the worry characteristic of GAD tends to be more frequent and longer in duration (Dupuy, 
Beaudoin, Rhéaume, Ladouceur, \& Dugas, 2001; Hoyer, Becker, \& Roth, 2001) as well as more uncontrollable and less realistic (Craske, Rapee, Jackel, \& Barlow, 1989). Individuals with GAD are also more likely to view their worry as dangerous (Ruscio \& Borkovec, 2004) and difficult to regulate compared to controls (Craske et al., 1989; Hoyer et al., 2001). GAD is one of the most common anxiety disorders, with a lifetime prevalence of between 4 - 6\% (Blazer, Hughes, George, Swartz \& Boyer, 1991; Kessler et al., 1994). Research indicates that GAD is among the most disabling of the anxiety disorders, and is comparable to physical illness and depression in terms of interference and distress (Maier et al., 2000; Wittchen, 2002). Studies show that individuals with GAD have higher consultation and comorbidity rates than individuals with other anxiety disorders (Dugas, Freeston, et al., 1998). GAD is also associated with an elevated use of health care services, and higher costs due to occupational disability, compared to the general population (Koerner et al., 2004; Wittchen, 2002). The inclusion of excessive worry as the cardinal feature of GAD in the DSM-III-R (American Psychiatric Association, 1987) led to numerous studies investigating the properties of worry and its underlying mechanisms. This research generated several theoretical models of excessive worry and GAD (e.g., Borkovec, Ray, \& Stöber, 1998; Dugas, Gagnon, Ladouceur, \& Freeston, 1998; Wells \& Carter, 1999), which in turn have led to empirically supported treatments (e.g., Borkovec \& Costello 1993; Borkovec, Newman, Pincus, \& Lytle, 2002; Dugas et al., 2010; Dugas et al., 2003; Ladouceur, Dugas, et al., 2000). Among clinical disorders, high levels of worry appear to be most characteristic of GAD (Andrews et al., 2010; Ladouceur et al., 1999; Ruscio, 2002); however, worry is also present in other 
anxiety disorders, and to some extent in mood disorders as well (Barlow, 1988; Riskind et al., 1991; Starcevic et al., 2007).

\section{Intolerance of Uncertainty}

Given that excessive worry is the core feature of GAD, and is moreover present in other anxiety disorders, worry itself has been the focus of several studies. The goal of some recent research has been to understand the cognitive processes that may drive and perpetuate excessive worry. A cognitive process that has received considerable research attention, and that has been specifically linked to excessive worry, is Intolerance of Uncertainty (IU). IU can be defined as a dispositional characteristic that results from a set of negative beliefs about uncertainty and its implications (Dugas \& Robichaud, 2007, p. 24). More specifically, individuals who are intolerant of uncertainty believe that uncertainty is stressful, unfair, interferes with their ability to function, and should be avoided (Sexton \& Dugas, 2009a). Negative reactions to uncertainty, and difficulty coping with uncertainty, can be seen as a type of dispositional bias which gives rise to excessive worry. It can be imagined that someone highly intolerant of uncertainty would encounter numerous instances of uncertainty in everyday life; negative beliefs about uncertainty (i.e., "Uncertainty makes me uneasy, anxious, or stressed"; "It's unfair having no guarantees in life") would increase their tendency to worry, even about minor events. $\mathrm{IU}$ is the key process in the theoretical model of worry proposed by Dugas, Gagnon, et al. (1998). In addition to IU, this model includes other components thought to be related to worry (e.g., negative problem orientation, cognitive avoidance, and positive beliefs about worry). While research has confirmed a link between all these processes and worry, IU 
appears to be the central mechanism that underlies the other processes, and is most specifically related to worry.

Research supports the proposal that IU is both highly and specifically related to worry. For example, research has shown that IU maintains a strong and significant relationship with worry, even when associated factors such as anxiety (Buhr \& Dugas, 2002), symptoms of depression (Buhr \& Dugas, 2002; Dugas, Schwartz, \& Francis, 2004), dysfunctional attitudes (Dugas, Schwartz, \& Francis, 2004), perceived sense of control, and perfectionism are statistically controlled (Buhr \& Dugas, 2006). In addition, IU has been found to accurately differentiate individuals with GAD from non-clinical controls, and from those with other anxiety disorders (Dugas, Marchand, \& Ladouceur, 2005; Ladouceur et al., 1999; note however that findings are less definitive with regards to Obsessive-Compulsive Disorder, i.e., Holaway, Heimberg, \& Coles, 2006). In clinical populations, IU successfully distinguishes mild GAD from moderate to severe GAD (Dugas et al., 2007). Moreover, experimental manipulations have shown that increasing IU leads to more worry, whereas decreasing IU leads to less worry (Ladouceur, Gosselin, \& Dugas, 2000).

\section{Intolerance of Uncertainty and Information Processing}

While the above research demonstrates a strong relationship between IU and worry, it does not explain the mechanism underlying this relationship. In other words, we know that IU contributes to worry, but we do not yet know how. Cognitive theory, with the aid of an information processing framework, allows us to explore just this type of question. Essentially, cognitive theory proposes that our basic beliefs or schemas affect how we process information in our environment (i.e., Beck \& Clark, 1997; Clark \& Beck, 
2010). The information processing approach examines processing in terms of different stages, such as attention, interpretation, and memory (see Bower, 2000, for an overview). Combined, these theories produce questions concerning how a belief such as IU might affect what worriers attend to, understand, and remember about the world around them. More specific questions might be: Do individuals high in IU pay more attention to certain types of information in their environment? How do they interpret and remember this information? How might IU influence different aspects of information processing, and how might this lead to increased levels of worry? Answers to these questions could enhance not only current cognitive models of excessive worry, but could also inform psychological treatments for disorders such as GAD.

Contemporary research does in fact suggest that IU can influence and direct information processing. For example, a recent study assessed whether individuals high in IU have an attentional bias toward words denoting uncertainty (Heinecke, Koerner, Dugas, \& Mogg, 2006). This study used a probe classification task, in which a probe (the letter E or F) replaced different types of stimulus words. Words were presented in pairs, comprised of one neutral and one other word, that was either physically threatening, socially threatening, uncertain, or positive. When a probe appeared, participants were asked to classify it as ' $\mathrm{E}$ ' or ' $\mathrm{F}$ ' by pressing a key; faster reaction times denoted an attentional bias toward the word that the probe replaced. The results showed that individuals high in IU were faster at classifying probes that replaced either uncertain or physically threatening words, indicating an attentional bias toward threatening and uncertain information. Unfortunately, no other studies to date have specifically examined the role of IU in attention. 
Other research suggests that IU influences the interpretation, or appraisal, of ambiguous information. For example, Dugas, Hedayati, et al. (2005) assessed the relationship between IU and the appraisal of scenarios using the Ambiguous/Unambiguous Situations Diary (AUSD; Davey et al., 1992). The AUSD is made up of a set of scenarios that describe everyday events. The scenarios of interest for this study were ambiguous in nature, for example, "Today, my supervisor called me to their office to discuss the change in the quality of my work over the last few weeks". Participants were asked to read these scenarios, imagine that the events had happened to them, and then rate their level of concern about the scenario. Higher levels of concern indicated that a scenario had been interpreted in a more negative way. The results showed that individuals high in IU reported significantly more concern about ambiguous scenarios compared to those low in IU. In addition, regression analyses showed that IU made a significant and unique contribution to the prediction of these negative appraisals, beyond demographics, mood, anxiety, and worry (Dugas, Hedayati, et al., 2005).

Expanding on this methodology, Koerner and Dugas (2008) examined appraisals in high and low IU participants, using a larger set of positive, negative, and ambiguous scenarios. They found that IU was the strongest predictor of concern about ambiguous scenarios when demographics, mood, and physical anxiety symptoms were controlled. In addition, a mediational analysis showed that appraisals of ambiguous scenarios partially mediated the relationship between IU and worry. Similar findings have emerged from studies using pictorial stimuli. For example, Koerner and Dugas (2007) recently conducted a study using neutral, ambiguous, negative, and positive pictures selected from the International Affective Pictures System (IAPS; Lang, Bradley, \& Cuthbert, 2001). 
Participants were asked to rate the pictures on pleasantness (this was used as an index of appraisals, such that 'very unpleasant' = negative, and 'very pleasant' = positive; see Finucane, Alhakami, Slovic, Johnson, 2000). As with the written scenario studies, the results showed that individuals high in IU rated negative and ambiguous pictures as significantly less pleasant compared to those low in IU. These findings are consistent with the idea that an information processing bias (i.e., a negative appraisal of ambiguity) may be one of the mechanisms by which a cognitive process such as IU ultimately leads to worry. These studies together support the proposal that IU is associated with negative interpretations of ambiguity. The tendency to negatively interpret ambiguity may lead high IU individuals to perceive more situations as threats, and consequently worry more.

Finally, some research suggests that there is a memory bias for uncertain information among individuals high in IU. In the same paper described above, Dugas, Hedayati, et al. (2005) carried out a second study examining the relationship between IU and memory for uncertain words. For this study, an incidental learning task was used; in other words, participants were not told that their memory will be tested, nor were they asked to memorize material. Instead, they performed a task that involved the stimuli, and were later tested on their memory for the stimuli. The value of incidental learning is that it not only resembles the type of learning that occurs in everyday life, but also has the capacity to reveal information processing biases. For example, if a certain type of information is better remembered following incidental learning, we can infer that there was preferential or biased processing of that information (i.e., Dalgleish, 1994). In the Dugas, Hedayati, et al. (2005) study, participants viewed a series of neutral and uncertain words projected on a screen, and rated each on its familiarity (the incidental learning 
task). Following the ratings, participants were given a free recall task. While all participants recalled more uncertain than neutral words, results showed that high IU participants recalled a significantly higher proportion of uncertain words (uncertain words/neutral words) than did the low IU participants.

Taken together, these studies suggest that individuals high in IU may have an attentional bias toward uncertain and threat words; a tendency to appraise uncertain and negative scenarios as more threatening; and an enhanced memory for uncertain words. The findings of a bias toward threat and negative information are not unexpected, given that these biases are characteristic of anxiety more generally, as will be discussed below. However, findings of a bias for uncertainty among high IU individuals may represent an information processing bias that is specific to worry. Overall, these findings are consistent with a view of IU as a cognitive process that influences all phases of information processing: attention, appraisal, and memory. If the results of these studies are upheld, they may well explain how IU leads to excessive worry. An individual high in IU, with enhanced attention and recall for uncertainty, and who interprets uncertainty in a negative way, might experience more cues to worry, and consequently worry more. However, given the very few studies to date that have directly examined this question, conclusions concerning IU and information processing must await further exploration. Although information processing studies of IU are scarce, there is a relatively large body of research concerning information processing biases in GAD, and some research concerning worry. Given the well-established relationship between IU, worry, and GAD, a review of this literature will provide additional clues concerning the potential role of IU in information processing. However, this research should be considered with 
several caveats in mind. First, most of the research comparing information processing in controls, high worriers, and GAD patients does not specifically measure IU. Therefore, any biases found in the GAD and high worry groups may be due to IU; conversely, biases may also be due to some other cognitive process, personality trait, or symptom that is common to these groups. Second, much of the research that will be reviewed concerns a bias for threat rather than for uncertainty. This focus on the processing of threat is based on Beck's (Beck \& Clark, 1997; Clark \& Beck, 2010) cognitive model of anxiety, which proposes that anxiety and the anxiety disorders are characterized by selective processing of threatening stimuli, at all stages of information processing. This framework has been influential and has shaped much information processing research on anxiety. Many studies have consequently employed threat stimuli that are broadly conceptualized (e.g., stimuli that represent threat in terms of physical harm) or undifferentiated (i.e., a mix of physically threatening, socially threatening, and depressogenic stimuli). While some studies have used stimuli intended to represent themes specific to GAD (e.g., Eysenck, Mogg, May, Richards, \& Mathews, 1991; also see review by Mogg \& Bradley, 2005), these distinctions are often omitted. The implications of using stimuli that have limited specificity for particular forms of anxiety, and for particular anxiety disorders, is an issue to which we will later return. For the time being, however, it should be noted that the definition of threat varies widely from study to study in the GAD/worry literature.

A third qualification concerns the distinction between GAD and non-GAD worry. While elevated worry characterizes both GAD patients and non-clinical high worriers, there are differences between these populations. For example, compared to non-clinical worriers, individuals with GAD appear to worry about a wider range of topics (Roemer, 
Molina, \& Borkovec, 1997) for longer durations (Craske et al.,1989), and report more distress and physical symptoms associated with worry (Hoyer et al., 2001; Ruscio \& Borkovec, 2004). Even when matched on level of trait worry, one study found that GAD worriers reported a greater subjective loss of control over worry, more negative intrusions following worry, and more negative beliefs about worrying, than did high-worry controls (Ruscio \& Borkovec, 2004). Despite these differences, there are many similarities between high worriers and individuals with GAD. For example, worry topics appear to be comparable across GAD and non-GAD groups (Craske et al., 1989; Dugas, Freeston, et al., 1995; Shadick, Roemer, Hopkins, \& Borkovec, 1991). Moreover, the actual experience of GAD and non-GAD worry may be quite similar in terms of unpleasantness, anxiety, and the attentional interference it produces (Ruscio \& Borkovec, 2004). Ruscio, Borkovec, and Ruscio (2001) further observe that the majority of findings from studies of non-clinical worry have been later replicated in clinical samples. Finally, two taxometric studies have examined the latent structure of worry, and both have found evidence that worry is dimensional in nature; in other words, non-clinical and clinical worry appear to exist on a continuum, rather than belonging to discrete categories (Olatunji, BromanFulks, Bergman, Green, \& Zlomke, 2010; Ruscio et al., 2001). Therefore, it seems appropriate to consider research on both non-clinical high worry and GAD, while acknowledging that certain differences limit the between-groups generalizability of findings.

\section{Information Processing in GAD and Worry}

Attentional biases. With the above qualifications in mind, a considerable body of research demonstrates an attentional bias for threatening information in GAD, which is 
less present in non-anxious individuals. Various methodologies have been used to explore this question. One of the most prevalent is the emotional or modified Stroop task, in which participants are asked to name the colour a word is printed in, rather than reading the word. Slower colour naming of threat compared to neutral words shows attentional bias toward the threatening meaning of the word, which slows colour naming (Williams, Mathews, \& MacLeod, 1996). Numerous studies using the emotional Stroop task have confirmed the presence of an attentional bias for threat in GAD patients, as compared to controls (Becker, Rinck, Margrath, \& Roth, 2001; Bradley, Mogg, Millar, \& White, 1995; Golombok et al., 1991; Martin, Williams, \& Clark, 1991; Mathews \& Klug, 1993; Mathews \& MacLeod, 1985; Mathews, Mogg, Kentish, \& Eysenck, 1995; Mogg, Bradley, Williams, \& Mathews, 1993; Mogg, Mathews, \& Weinman, 1989). In one study, while colour-naming latency was not biased, speed and direction of eye movements indicated an attentional bias for threat among GAD participants (Mogg, Millar, \& Bradley, 2000). In addition, attentional bias for threat can be found with the emotional Stroop even when awareness of the stimuli are restricted by a backward mask (e.g., a random letter or number string following a brief presentation of the word; Bradley, Mogg, Millar, et al., 1995; Mogg et al., 1993).

Other widely-used attentional tasks include the dot-probe and probe classification tasks. In these tasks, probes replace neutral and threat stimuli in different locations on a visual display; an attentional bias for threat is indicated by faster identification of probes that replace threat stimuli (MacLeod, Mathews, \& Tata, 1986). Several dotprobe/classification studies using words as stimuli have found evidence of attentional bias for threat among individuals with GAD (MacLeod et al., 1986; Mathews, Ridgeway, 
\& Williamson, 1996; Mogg, Bradley, \& Williams, 1995; Mogg, Mathews, \& Eysenck, 1992; Taghavi, Neshat-Doost, Moradi, Yule, \& Dalgleish, 1999). Studies using backward masking have produced similar results (Mogg et al., 1995), as have studies using threatening faces (Bradley, Mogg, White, Groom, \& de Bono, 1999).

Evidence of an attentional bias in GAD has also emerged from visual search paradigms. In a visual search task, participants are asked to identify a threat stimulus presented within a field of neutral stimuli; faster reaction time denotes an attentional bias for threat. Conversely, slower identification of a neutral stimulus within a field of threat stimuli is a measure of difficulty disengaging from threat - another manifestation of attentional bias. Visual search tasks have provided some support for both an attentional bias, and difficulty disengaging from threat words in GAD (Rinck, Becker, Kellerman, \& Roth, 2003). A modified version of this paradigm showed that GAD participants were significantly slower to identify targets in the presence of threatening as compared to neutral distractors (Mathews, May, Mogg, \& Eysenck, 1990).

Taken together, the studies reviewed above provide considerable evidence of an attentional bias for threat in GAD, which converges across tasks, and suggests that this is a robust effect. In support of this conclusion, a recent meta-analysis found a moderately large effect size across studies of attentional bias in GAD (Cohen's $d=.56$; Bar-Haim, Lamy, Pergamin, Bakermans-Kranenburg, \& van Ijzendoorn, 2007).

Interpretation biases. Research also suggests that there is a relationship between GAD, worry, and negative interpretations of ambiguity. A widely-used test of interpretation bias involves homophones. In this type of task, a series of words are presented auditorily and participants are asked to write them down. Among the words 
presented are homophones: words with one pronunciation but two different meanings, such as pane/pain or dye/die. One study using this task found that participants with GAD reported a significantly higher number of threatening meanings; in other words, GAD patients made more threatening interpretations of ambiguous words (Mathews, Richards, \& Eysenck, 1989).

Another method used to assess interpretation bias in GAD involves the presentation of ambiguous text. In this type of procedure, a series of sentences are presented, some of which are ambiguous (i.e., "The two men discussed the best way to blow up the boat"). Participants are later shown a series of new sentences and are asked to decide whether they have the same meaning as any previously viewed sentence. The possible responses included an equal number of disambiguations that are either threatening (i.e., "The men talked about how they should destroy the boat") or nonthreatening ("The men talked about how they should inflate the boat"). Two related studies by Eysenck and colleagues (1991) showed that compared to controls, GAD patients more often chose threatening disambiguations of sentences (Eysenck et al., 1991).

Further support for an interpretative bias in GAD comes from studies that have experimentally manipulated these biases. Innovative research has shown that participants can be trained to make non-threatening interpretations of ambiguity. In two such studies, interpretation training had two phases. In the first phase, participants were visually presented with homophones (e.g., patient), followed by a semantically-related word fragment to complete. Participants were assigned to groups that were given either benign word fragments (e.g., kind) or a control group that received an equal number of benign 
and threatening fragments (e.g., sick; kind). In the second training phase, participants heard a scenario that remained ambiguous until it was completed by a final benign or threatening word; (e.g., “...you think that your boss will put it down to you being busy/lazy"). Participants in the benign condition received only benign completions, whereas those in the control condition received an equal number of benign and threatening completions. Using these methods, one study showed that following benign interpretation training, high worriers experienced fewer negative thought intrusions and anxiety, compared to high worriers in the control condition (Hirsch, Hayes, \& Mathews, 2009). These findings were then replicated in a group of patients with GAD, which further confirmed that after the benign training, patients made fewer threatening completions of ambiguous sentences (Hayes, Hirsch, Krebs, \& Mathews, 2010). These and the studies reviewed above suggest a significant relationship between interpretive biases, worry, and GAD.

Memory biases. Among GAD patients and high worriers, biases in attention and interpretation have now been fairly well established (see reviews by MacLeod \& Rutherford, 2004; and Mathews \& MacLeod, 2005). In contrast, research on memory bias is inconclusive. This is a puzzling discrepancy: if GAD patients and high worriers have enhanced attention for threat, and a tendency to make threatening interpretations, this should be reflected in the increased availability of threat-related information in memory. However, studies of memory bias in GAD and worry have produced a series of contradictory findings.

Adding to the complexity of this research is that both explicit and implicit memory have been explored. Explicit memory is generally defined as a conscious attempt 
to retrieve information from memory; for example, the attempt to remember one's grocery list. In contrast, implicit memory does not involve effortful retrieval, but instead is shown through enhanced performance on a task due to prior experience with certain information; for example, faster completion of food-related words in a crossword puzzle, although one's grocery list is forgotten (see Roediger \& Amir, 2005, for an overview). While certain tasks have traditionally been used to measure each type of memory, tasks are not intrinsically measures of either explicit or implicit memory: this rather depends on the instructions given to participants. Completion of word stems, for example, has frequently been used to test implicit memory. In a typical word stem procedure, participants perform an incidental task with a set of word stimuli. Subsequently, they are instructed to complete a series of word stems with the first word that comes to mind. The word stems are derived from both new (unprimed) and previously viewed (primed) words. The measure of implicit memory is the degree of superior performance on primed compared to unprimed word stems; as this does not involve effortful retrieval of information from memory, it can be considered a test of implicit memory. Conversely, using the same procedure, participants can be asked to complete word stems using the stimuli they previously viewed. As this involves effortful retrieval of information, it can be considered an explicit test of memory. These distinctions between explicit and implicit memory will be applied to the review of research that follows. Finally, unless otherwise specified, all the studies reviewed below use an incidental learning approach.

Studies of explicit memory. Much of the early information processing research on worry and GAD focussed on explicit memory. In addition, many studies used a selfreferent procedure, which requires participants to associate stimuli with themselves in 
some way during the learning phase (e.g., rate the extent to which each word describes you). It seems apparent that self-referenced material should be more salient to participants, and therefore better remembered. In fact, the superiority of the self-reference effect on memory has been well-established in non-clinical populations (see Symons \& Johnson, 1997, for a review). Consequently, this approach was often used in studies of GAD and worry.

In one early study, Mogg, Mathews, and Weinman (1987) explored explicit memory in controls and generally anxious patients; the patients were diagnosed with anxiety state according to the International Classification of Diseases, 9th revision (ICD9-R: U.S. Department of Health and Human Services, 1980). In this study, participants heard a mixed set of adjectives, half of which were positive (e.g., amused, secure). The remaining adjectives were either negative and socially or physically threatening (humiliated, trapped), or negative and non-threatening (bored, gloomy). In a procedure often used in this type of research, candidate stimuli were generated by the researchers, and a group of expert judges then reached consensus on stimuli that optimally represented each of the categories of interest. As is also typical, word norms were consulted (e.g., Thorndike \& Lorge, 1944) to ensure that words were equivalent in their frequency of use; words were also matched as closely as possible in length. Before each word was presented, participants were instructed to refer the word to themselves (does this word describe you?) or to a well-known British television personality (does this word describe Angela Rippon?); these represented self- and other-referent conditions, respectively. Following a $20 \mathrm{~s}$ distractor task to reduce recency effects, participants were asked to write down as many words as they could remember (i.e., a free recall test of 
explicit memory). Subsequently, they were given a sheet containing the words they had heard, and a matched set of new words; they were asked to identify the words they had heard (a recognition test of explicit memory). Finally, participants completed self-report questionnaires measuring trait anxiety, state anxiety, depression, and vocabulary. The results showed that positive words and self-referenced words were better remembered overall. However, there was no difference in the number of threatening words recalled in the anxious and control groups. In fact, there was a trend for anxious participants to recall fewer threat words than controls. Measures of recognition memory (hit rate, sensitivity, and response bias) produced similar results. Interestingly, the researchers found that several anxious patients reported elevated levels of depression, although none had a comorbid diagnosis of depression. While this is not surprising given the high co-occurrence of anxiety and depression (i.e., Brown, Campbell, Lehman, Grisham, \& Mancill, 2001), it does raise the question of how depressed mood may affect this type of task. Based on their findings, the authors proposed that anxiety may be characterized by an attentional bias for threat, but not a memory bias. The authors also noted previous research suggesting that a memory bias may be characteristic of depression; they proposed that the same pattern may not hold for anxiety.

Using a similar methodology, Mogg and Mathews (1990) instructed controls and generally anxious patients (diagnosis of anxiety state; ICD-9-R, 1980) to refer half of a set of words to themselves (does this word describe you? ) and the rest to other people (does this word describe the average person on the street?). Words with self-and otherreferent instructions were randomly mixed together. In this study, however, words represented either an anxious state (e.g., nervous, afraid) or a non-anxious state (secure, 
carefree); these were selected by judges and matched on frequency. Participants were warned that their memory for the words would later be tested. Results of a free recall test showed the expected advantage for self-referenced words. In addition, anxious patients recalled significantly more anxious than non-anxious words compared to controls; however, this was not specific to self-referenced words. When the researchers analyzed intrusions on the recall test ("recalled" words that had not actually been presented) they found that anxious participants had significantly more anxiety-related intrusions than controls. They therefore concluded that their results were more likely due to a response bias for anxious stimuli, than to a memory bias. A further caveat is that participants were warned that their memory would be tested. The results may therefore demonstrate that anxious patients are better at memorizing anxious words, or that asking them to memorize words creates a response bias toward anxious information. In either case, the results of this study are difficult to integrate with those that use an incidental learning approach.

Becker, Roth, Andrich, and Margraf (1999) also employed a self-referent task, comparing controls to patients diagnosed with GAD or Social Phobia according to the Diagnostic and Statistical Manual of Mental Disorders (3rd ed., revised; American Psychiatric Association, 1987). The words used in this study were either GAD-related (e.g., injury, nervous), speech-related (talk, blush), positive (dreaming, flower), or neutral (chair, umbrella). The stimuli used in this study were drawn from a larger pool of words generated by a group of anxiety experts; selected words were then matched on frequency. Stimuli were presented in random order on a computer screen, and participants were asked to imagine a visual scene related to the word and themselves. Participants then 
completed a free recall test and self-report questionnaires. The results showed that positive words were better recalled by all groups. However, there were no differences in the number of GAD or speech-related words recalled by any group. Of further interest is that participants were also asked to rate the word stimuli following testing. Analyses showed that while GAD patients rated the GAD words as more relevant and unpleasant than did controls, patients with Social Phobia also rated GAD words as highly relevant and unpleasant, suggesting that the stimuli may have had limited specificity to GAD.

Other researchers eschewed the self-referent approach, in part due to the nonsignificant findings of some previous studies. Mathews and MacLeod (1986), for example, administered a dichotic listening task to controls and GAD patients (DSM-III, 1980). In this study, participants wore headphones and listened to a story narrated in one ear. In the other ear, a series of words were periodically heard, which they were told to ignore. Simultaneously, participants visually monitored a computer screen, and pressed a key in response to the appearance of a probe. The words heard in the unattended ear were an equal mix of threat words (e.g., timid, emergency, worried) and non-threat words (playful, prize, friend). Word stimuli were created for this study by the researchers, and matched using frequency norms. As attentional biases have been found when stimuli are outside of awareness (i.e., with quickly presented or backward-masked stimuli), Mathews and MacLeod used a very brief "pre-attentive" exposure for words in the unattended channel. Before the dichotic listening task, participants completed a set of self-report questionnaires. As expected, the researchers found that when threat words were in the unattended channel, GAD patients were significantly slower to identify probes. However, a subsequent recognition test showed no indication of awareness for the unattended 
words in controls or GAD patients, and no memory bias for threat words in the GAD group. While self-reported depression was higher in the GAD group, no significant correlation was found between task reaction times and depression, leading the authors to conclude that their findings were probably due to anxiety and not depression. Based on their results, the researchers suggested that biases in anxiety may concern the preferential allocation of processing resources toward threat at an early stage of processing (i.e., a pre-attentive bias), rather than a memory bias for threat.

Using the emotional Stroop paradigm, Mogg and colleagues (1989) explored memory bias in controls and a group of generally anxious patients (diagnosis of anxiety state; ICD-9-R, 1980). In this study, an equal number of words were physically threatening (e.g., disease, mutilated), socially threatening (failure, inadequate), and nonthreatening (holiday, contented). Most of these stimuli were obtained from those used in previous research ( i.e., Mathews \& MacLeod, 1985). In standard Stroop fashion, participants viewed the words in random order and named the ink colour of each word. Subsequently, they completed a test of recognition memory, and then reported whether their most typical worries were physical or social in nature. They also completed a package of self-report questionnaires. The results showed the expected emotional Stroop effect, with significantly slower colour naming of threat words by GAD patients. In addition, patients with predominantly physical worries were significantly slower at colour naming physical threat as compared to non-threat words, suggesting specificity of the physical threat stimuli. However, a similar pattern with regards to social worries and social threat words did not reach significance. Most notably, however, there was no difference between the groups in recognition memory (sensitivity) for threat words. 
Analyses of the self-report measures showed a significant relationship between threat word interference and levels of both trait anxiety and depression. The researchers observed that the lack of memory bias in this study is only puzzling if we assume that biases must operate in the same way across all stages of processing. As an alternative, they proposed a model in which there is a bias for threat in attentive processing, but not in subsequent elaboration; this type of model would be consistent with biased attention for threat, but not biased memory.

In one of the few studies that found an explicit memory bias in GAD, Friedman, Thayer, and Borkovec (2000) employed a classical conditioning paradigm. In their study, control and GAD participants (DSM-III-R, 1987) viewed a series of words paired with neutral stimuli (coloured dots, used to evaluate higher-order conditioning, and not related to this study). The words that participants viewed included mixed physical threat words, social threat words, and non-threat words, derived from previous research (i.e., Mathews \& MacLeod, 1986). Participants were asked to read each word silently to themselves as it appeared on the screen. They were then given a free recall test and completed self-report measures. This procedure was repeated in two studies, each with a different sample of GAD patients and controls. The results showed that in both studies, GAD patients recalled significantly more threatening words than did controls; moreover, within the GAD groups, more threat than neutral words were recalled. The researchers suggested that the $8 \mathrm{~s}$ exposure to the stimuli (longer than most studies) may have contributed to conscious processing and hence memory for threat. They also speculated that selfreferent tasks may have led to non-significant findings in other studies. Specifically, they proposed that imagining the self in regards to threat may be too stressful for anxious 
patients, who may ultimately avoid thoughts about the threat (i.e., they may engage in cognitive avoidance); this would reduce the processing of threat and subsequent memory.

Studies of implicit memory. In the dearth of explicit memory findings, researchers began to focus on the question of biases in implicit memory. As with studies of explicit memory, self-referent tasks were often used, with varying results. In most of the studies that follow, explicit memory was also measured. Interestingly, an implicit memory bias, but not an explicit memory bias, was sometimes found within the same study.

Bradley, Mogg, and Williams (1995) used a lexical decision task to assess implicit memory, and a self-referent task with free recall for explicit memory. Their participants included controls and patients with either GAD or Major Depression (DSMIII-R, 1987). In the first phase of the lexical decision procedure, participants rated a set of stimulus words on the frequency with which they used them. There were mixed equal numbers of words that were anxious (e.g., embarrassed, cancer), depressed (pessimistic, despair), positive (bliss, adorable), and neutral (doorbell, carpet). The words were based on a pool of words used in previous research (i.e., Mathews, Mogg, May, \& Eysenck, 1989, and others), and were selected based on the consensus of three judges. After the word ratings, the lexical decision task was administered, in which a series of individual stimuli appeared on the computer screen. Some of the words were those participants had previously rated (primed stimulus words), some were new words (unprimed stimulus words), and others were pronounceable non-words (e.g., traib, enpine). Participants were asked to decide whether or not the letters on the screen formed a word. The measure of implicit memory was the speed at which these lexical decisions were made: faster 
decisions were expected for primed words, and particularly for primed words that had been preferentially processed (i.e., anxious or depressed words). In order to explore any effects that might occur outside of awareness, some of the lexical decision trials were sub-threshold presentations (i.e., presented for only $14 \mathrm{~ms}$ and followed by a string of mixed letters to visually mask the word). In the explicit memory portion of the experiment, participants viewed the set of unprimed words from the lexical decision task, and were asked to rate each word on how relevant it was to their personal concerns. They then completed a free recall test and self-report measures. For the lexical decision task, priming scores were calculated (unprimed word latencies minus primed word latencies), in order to control for baseline performance. Results showed that while there was a significant priming effect overall (faster decisions for primed words), there was no difference between the GAD and control groups on decisions for different types of words, on normal or sub-threshold trials. Furthermore, there was no evidence of a GAD group bias toward any type of word on the free recall task. There was therefore no evidence of either an implicit or explicit memory bias in GAD patients. However, there was a significant bias for priming of depressed words in the depressed group, in both normal and sub-threshold trials; a similar bias for depressed words was found on the free recall task. While the absence of an explicit memory bias in the GAD group is consistent with previous studies, the authors observed that a finding of implicit memory bias in depression is unusual. However, they noted that this study underscores the differences between anxiety and depression, as depression may be characterized by both implicit and explicit memory biases, whereas anxiety may not. 
Otto and colleagues used a dichotic listening task to assess the role of hemispheric laterality in explicit and implicit memory (Otto, McNally, Pollack, Chen, \& Rosenbaum, 1994). To assess hemispheric laterality, controls and patients with GAD or Panic Disorder (DSM-III-R, 1987) completed a dichotic listening task in which a series of similar neutral word pairs were heard, one word in each ear (e.g., bar, car). Participants were asked to write down the word they heard (in this procedure only one word is usually perceived). An incidental learning task followed, in which participants viewed a new set of stimulus words and were asked to rate each word on its personal emotional significance. The stimuli were mixed and included an equal number of panic (e.g., suffocate), general threat (cancer), positive (creative), or neutral (carpet) words. These stimuli were primarily derived from previous research (i.e., Mathews, Mogg, et al., 1989, and others), and were matched in terms of frequency. Participants then completed two memory tests using word stems: for half of the stimuli, an implicit test (i.e., complete the stems with any word that comes to mind), and for the other half, an explicit test (i.e., complete the stems with the words you rated earlier). The results showed no differences between the groups in implicit memory for different types of words. On the explicit test, neutral words were most poorly recalled, but there were also no differences between the groups. Dichotic listening responses were then analyzed to identify the ear in which more words were correctly identified; this was used as an index of perceptual asymmetry. Perceptual asymmetry (PA) was used as a measure of relative hemispheric activation, with left-ear PA being an indication of right hemisphere lateralization, and right-ear PA of left hemisphere lateralization. This was of interest as previous research has found a relationship between right hemisphere lateralization and indices of negative affect and 
behavioural withdrawal (see Otto et al., 1994, for a review). In this study, however, the results showed no PA difference between the anxious and control groups. However, regression analyses suggested that a right-ear PA (left-hemisphere lateralization) in GAD and Panic patients was predictive of a greater explicit memory bias for general threat and panic words. The researchers suggested that anxious participants with left-hemisphere lateralization may better process verbal information, and therefore show a clearer explicit memory bias for threatening word stimuli.

Mathews, Mogg, and colleagues (1989) found an implicit but not an explicit memory bias for threat in a group of GAD patients. In this study, controls and GAD patients (DSM-III-R, 1987) were presented with a series of words, and were asked to imagine a scene involving themselves and the word, and then rate its pleasantness. The words participants viewed were comprised of mixed equal numbers of threat and nonthreat words. Threat words included social threat (e.g., lonely, insult) and physical threat words (cancer, collapse); non-threat words included positive (confident, bliss) and neutral words (emblem, fountain). The word stimuli were based on previous research (MacLeod et al., 1986; Mathews \& MacLeod, 1985) and were matched on both frequency and length. During the imagery task, participants viewed a proportion of the total stimuli, with the rest retained as unprimed stimuli for memory testing. After the imagery task, participants were asked to complete two sets of word stems. Specifically, for the explicit memory test, participants were asked to complete one set of word stems using the words they had viewed. For the implicit test, participants were asked to complete the remaining set of word stems with the first word that came to mind. For both explicit and implicit word stem completion, half of the stems were for previously viewed 
primed words, and half for new unprimed words. All participants completed both tests, in counterbalanced order, and then filled out self-report questionnaires. Results did not show any explicit memory differences between the groups. For the implicit task, priming scores were calculated (primed minus unprimed stem completions) and showed a significant overall priming effect. In addition, control participants demonstrated a significant bias toward non-threat words. More importantly, however, the GAD patients showed a significant bias for threat words compared to controls, indicating an implicit memory bias for threat. Correlations did not show any significant relationships between word completion scores and self-reported anxiety or depression. Given these findings, the authors noted that a lack of explicit memory bias for threat would be consistent with the avoidance of conscious processing of threat by anxious patients. They proposed that the implicit bias found in this study may reflect a different kind of processing that may only emerge on implicit tests of memory.

Mathews and colleagues (1995) evaluated processing biases in a group of controls and GAD patients (DSM-III-R, 1987). The stimulus words used in this study were largely taken from the study described above; they included threat words (social and physical), and non-threat words (positive and neutral; see Mathews, Mogg, et al., 1989). The stimuli were divided into matched sets, with mixed equal numbers of threat and non-threat words. All participants in this study performed three tasks, each using a different word set; a fourth word set was not used, and was retained for memory testing. The first task was an emotional Stroop, in which participants named the colours of stimulus words. The second task was an attentional search, in which participants responded to a visual probe by pressing a key, while ignoring any words that appeared on the screen (i.e., distractors 
that were stimulus words). In the third task, participants viewed a set of word stimuli and counted the number of es in each word. After the letter counting, participants were asked to complete an implicit word stem task, for primed and unprimed words. Participants completed all tasks in counterbalanced order, and then filled out a set of questionnaires. The results showed the expected emotional Stroop effect, with slower colour-naming of threat words by GAD patients. In addition, GAD patients, compared to controls, showed significantly slower responses to targets that were accompanied by threatening distractors. While a significant overall priming effect was found on the word-stem task, there was no difference between GAD patients and controls in their completion of threatening words. Therefore, while attentional biases were present, no corresponding implicit memory bias for threat emerged in the GAD group. As these findings contrasted with those of Mathews, Mogg, et al. (1989), the authors highlighted a key difference between these studies: Mathews, Mogg et al. used a self-referent imagery task, while this study used letter counting. They noted that a self-referent imagery task is both elaborative and conceptual in nature, which, according to prevalent theories, should result in enhanced explicit memory (i.e., Roediger, 1990). In contrast, a letter-counting task should encourage encoding of perceptual features, and thereby enhance implicit memory. In light of their non-significant implicit memory findings, however, they speculated that the test they used - word-stems - may rely on more explicit, elaborative processes than is traditionally supposed; this could explain their lack of implicit memory findings. Another group of researchers attempted to replicate the findings of Mathews, Mogg, and colleagues (1989), but with different tasks. In their study, MacLeod and McLaughlin (1995) used stimuli made up of equal numbers of threat words (e.g., 
violence, disaster, humiliated) and non-threat words (symphony, superior, celebrates). Words were generated by the researchers, who then asked five clinical psychologists to rate the words on scale ranging from $1=$ non-threatening to $5=$ threatening. Stimuli were selected on the basis of these ratings, with subsequent $t$-tests confirming that threat and non-threat words differed significantly. Stimuli were matched for length and frequency, and placed in randomized order. The stimuli were divided into sets so that participants viewed some of the words during encoding (a Stroop task), and the remainder in two memory tests. The researchers asked controls and GAD patients (DSMIII-R, 1987) to complete a series of tasks, beginning with a calibration procedure (described below). Participants then completed an emotional Stroop task, in which they were asked to name the ink colour, but also read each word aloud. An explicit recognition memory test followed, using previously viewed and new stimuli. Due to concerns about the validity of word stems as an implicit memory task, the researchers instead employed a tachistoscopic identification procedure. In a tachistoscopic identification task, words are very briefly flashed on a computer screen. Implicit memory can be demonstrated if rates of correct identification are higher for primed than unprimed words. For this to be effective, however, there must be an appropriate display duration: if words are presented too quickly, none will be identified; too slowly, and a ceiling effect will obscure any priming effects. To this end, calibration was performed for each participant, in which neutral test words were presented at increasingly short durations. The duration at which each participant could accurately identify $50 \%$ of the words was then used for testing that participant. The third task in this study was the tachistoscopic identification, in which words viewed during the Stroop were mixed with new stimulus words (i.e., not used in 
any other task). Participants were asked to read each word aloud as it appeared on the screen. All participants completed both memory tasks, in counter-balanced order, and then self-report questionnaires. The results showed that while all participants had better explicit memory for the words they viewed during the Stroop, there was no difference between the groups. Responses to the implicit tachistoscopic task showed a significant overall priming effect. Moreover, compared to controls, GAD patients had a significantly higher priming score for threat compared to non-threat words. The results of this study are therefore consistent with those of Mathews, Mogg, et al. (1989) in that no explicit memory bias, but an implicit memory bias, was found among GAD patients.

Coles, Turk, and Heimberg (2007) took an individualized approach to the assessment of memory bias in GAD. Specifically, prior to testing, they presented a group of GAD patients (DSM-IV, 1994) with a large pool of potential stimulus words, and asked them to rate each word on its personal relevance (using a scale ranging from extremely negative for me to extremely positive for me). The words that each participant rated as most relevant were then used as stimuli for that person. The pool of potential words was specific to GAD: threat words representing common worry domains (e.g., emergency, bills, deadline), and positive words that were the opposite of the threat words (raise, relaxed, vacation). Neutral words were generated from several categories in order to match the different types of words in the other sets. Based on the ratings, a unique matched set of threat, positive, and neutral words was selected for each participant. One week after stimulus word selection, participants returned to the laboratory. Each GAD patient was experimentally yoked with a control participant, who was also tested with that patient's stimuli. Participants then viewed part of the stimulus set, and were asked to 
imagine a scene incorporating themselves and each word; they then rated the ease of generating each image. After completing a filler task, they were asked to complete an implicit word stem task for primed and unprimed stimulus words. Participants were then given a free recall task and questionnaires to complete. Results showed no significant differences between the groups on the explicit memory task. However, the implicit word stem completions showed that GAD patients completed significantly more threat words than did controls. Interestingly, there was no difference in the ease of imagery between the GAD and control groups. As a memory bias emerged when GAD patients were given stimuli that were relevant to their worries, the authors proposed that poor stimulus relevance may account for the lack of findings in other studies. Of further interest is that this study was the only one reviewed to include a self-report measure of worry; regressions showed that worry continued to make a significant contribution to implicit memory for threat words, above and beyond depression.

In summary, eleven of the twelve studies reviewed above included a measure of explicit memory. Only two of these eleven studies found an explicit memory bias, and in one case the findings are difficult to interpret, as participants were warned their memory would be tested (i.e., Mogg \& Mathews, 1990). In the other study that found an explicit memory bias, however, the effect was replicated in two separate samples (i.e., Friedman et al., 2000). Nevertheless, current research support for an explicit memory bias in GAD appears weak. In contrast, six of the reviewed studies measured implicit memory, and of these three produced significant findings. Support for an implicit memory bias for threat in GAD therefore appears to be equally divided. Interestingly, in all three studies that found an implicit bias, no corresponding explicit bias was found (i.e., Coles et al., 2007; 
MacLeod \& McLaughlin, 1995; Mathews, Mogg, et al., 1989). Of further note is that self-referenced tasks do not appear to be consistently related to a memory advantage in these studies. Specifically, six of the reviewed studies used some form of self-referenced task; of these, two found an implicit memory bias, and one an explicit memory bias. As a whole, therefore, research findings in this area appear too equivocal for definite conclusions to be drawn.

\section{Information Processing in High Trait Anxiety}

Given the mixed state of findings on memory bias in worry and GAD, it may be useful to consult a related body of research: that concerning trait anxiety. Trait anxiety can be defined as the relatively stable tendency to experience anxiety, and to respond fearfully to aversive stimuli (Spielberger, 1985). Research shows that there is a strong relationship between trait anxiety and worry (Borkovec et al., 1983; Brown, Chorpita, \& Barlow, 1998), and between trait anxiety and GAD (Rapee, 1991). Some researchers have proposed that high trait anxiety is a risk factor for the development of GAD (Eysenck, 1992; MacLeod \& Rutherford, 2004). Therefore, research on high trait anxiety may provide some insights about findings in worry and GAD.

In fact, information processing research on high trait anxiety closely mirrors research on worry and GAD. For example, numerous studies have found that high trait anxious individuals show an attentional bias for threat; as with GAD, this has been demonstrated with tasks such as the dot-probe, the emotional Stroop, and selective attention for threatening faces. Similarly, an interpretive bias for threat among trait anxious individuals has emerged from studies using homophones, lexical decisions, and the reading of ambiguous text (see reviews by Macleod \& Rutherford, 2004; Mathews \& 
MacLeod, 2005). Furthermore, high trait anxious individuals have undergone interpretive training, and results suggest that benign training can reduce both anxiety and the tendency to make negative interpretations (Mathews \& MacLeod, 2002; Mathews, Ridgeway, Cook, \& Yiend, 2007).

As with GAD and worry, however, findings begin to diverge when we consider memory. In parallel with research on GAD, both explicit and implicit memory have been explored; similarly, self referent tasks and word stimuli (threat versus non-threat) predominate. For example, a number of studies have used a variation of the self-reference procedure, in which participants refer half of the stimuli to themselves, and the remainder to other people. Martin, Ward, and Clark (1983) asked participants to rate how well a series of words described themselves, and the rest on how well they described the average undergraduate. They found that high trait anxious participants recalled significantly more negative self-referent adjectives than did those low in trait anxiety; moreover, the relationship between explicit memory for negative words and trait anxiety remained significant when depression was statistically controlled. Using a similar approach, Reidy and Richards (1997a) asked participants to refer words either to themselves or to a television personality; they found a free recall bias for threat words among participants highest in trait anxiety. In addition, they found that all participants remembered more positive words and more self-referenced words. In a replication study controlling for recency effects, similar findings emerged (Reidy \& Richards, 1997b). In another variation, Green and McKenna (1996) asked participants to judge whether word stimuli made semantically correct completions to a series of sentences which were self- 
or other-referent. They found no differences between high and low trait anxious groups in free recall of negative words.

Other studies have contrasted some form of self-reference with a read-only condition. For example, Richards and French (1991) created two experimental conditions: self-referenced imagery (i.e., imagine a scene concerning yourself and the word), and read-only (simply read and study the word). They then administered either an implicit word stem task or a free recall task. While there were no differences on the recall task, they found an implicit bias for threat words among high trait anxious participants, but only in the self-referent condition. In a similar study, Harrison and Turpin (2003) found an implicit memory bias for non-threat words across all participants on a wordstem completion task; however, no bias for threat emerged in the high trait anxious group. Russo, Fox, and Bowles (1999) also used self-referent imagery and read-only tasks at encoding, but tested implicit memory with either word stems or tachistoscopic word identification. In three studies that varied aspects of the procedure, they found no evidence of an implicit bias for threat words. In a simplified version of this method, Oldenburg, Lundh, and Kivisto (2002) used self-referent imagery alone at encoding, and then asked participants to complete a set of explicit word stems for half of the stimuli, and implicit word stems for the other half. Neither measure indicated a memory bias for threat among high trait anxious participants.

Another approach has been to create a task that requires participants to actually generate the stimuli during encoding. In one such study, Eysenck and Byrne (1994) gave participants letters or short phrases to complete; in each case, there was only one correct completion, which was a stimulus word. In this way, participants generated half of the 
stimulus set during the task; the other half were presented in read-only format.

Participants then completed implicit word stems, cued recall, and free recall tests. Results showed that high trait anxious participants had an explicit (cued recall) bias for threat in the generate condition, and an implicit bias for threat in the read only condition. In another variation, Dalgleish (1994) used a generate procedure in which participants were asked to solve anagrams of the stimulus words; this was followed by an explicit recognition test. In his second study, Dalgleish used a homophone task, followed by a free recall test. In neither study did high trait anxious participants show an explicit memory bias for threat.

Other research on trait anxiety has used the emotional Stroop task for incidental learning. For example, Mathews and MacLeod (1985) followed a Stroop task with a recognition test of explicit memory, and found no bias for threat words among high trait anxious participants. Richards and colleagues (1999) also used a Stroop task, followed by tachistoscopic word identification; in three different Stroop variations (colour naming; word naming; word and colour naming) results showed no differences between high and low trait anxious participants, although there was better implicit memory for threat words overall (Richards, French, Adams, Eldridge, \& Papadopolou, 1999). Using a different approach, Reidy (2004) used a list of non-worry and worry topics as stimuli, asking high and low trait anxious participants to rate how often they thought about each. He subsequently found no free recall bias for worry topics among high trait anxious participants. Among low anxious participants, however, non-worry topics were better recalled. In two other studies, Nugent and Mineka (1994) asked participants to rate their level of like or dislike for a series of words. This was followed by implicit word stems, 
recognition, and free recall tests. In one study, trait anxious participants recalled more threat words; however, this was not replicated in their second study. In addition, no recognition or implicit memory biases for threat emerged in either study.

In summary, while research supports attention and interpretation biases for threat in high trait anxious participants, memory findings are mixed. As many of the papers reviewed above included more than one test of memory, the findings will be summarized in terms of memory outcomes. In the papers reviewed, 30 separate indices of memory were calculated. Of these, 19 assessed explicit memory, and $6(32 \%)$ revealed a significant memory bias. The remaining 11 were implicit measures of memory, and 2 of these (18\%) showed significant biases. In total, therefore, six instances of explicit and two instances of implicit memory bias emerged from this research. In contrast to research on GAD, research on trait anxiety therefore appears to offer more support for an explicit memory bias. However, the number of studies examining explicit memory is nearly double those examining implicit memory, making it difficult to draw conclusions about implicit memory. Of further interest is that many of the significant findings emerged from self-referent paradigms, which also contrasts with findings on GAD and worry. What is consistent across research on trait anxiety, worry, and GAD, however, is the mixed state of evidence for a memory bias, compared to clear support for biases in attention and interpretation.

\section{Information Processing in Other Anxiety Disorders}

As previously noted, Beck’s (Beck \& Clark, 1997; Clark \& Beck, 2010) model predicts that a bias for threat should be present in all stages of information processing, across all of the anxiety disorders. A detailed review of information processing research 
for each of the anxiety disorders is beyond the scope of the current research. However, in order to provide context for the research already reviewed, a few key points from this research will be highlighted. Information processing research has in fact provided evidence of selective processing for threat in each of the anxiety disorders. However, the type of biases vary from disorder to disorder, as do the consistency of results. For example, evidence of attention bias for threat appears to be clearest for GAD, followed by Post-traumatic Stress Disorder(PTSD) and Panic Disorder (PD); findings in Social Phobia (SP) and Obsessive-Compulsive Disorder (OCD) are mixed. Conversely, support for biased interpretations appear strongest in GAD, SP and PD, with less conclusive evidence for the other anxiety disorders (see reviews by Buckley, Blanchard, \& Neill, 2000; Casey, Oei, \& Newcombe, 2004; Coles \& Heimberg, 2002; Hirsch \& Clark, 2004; McNally, 1994; Muller \& Roberts, 2005). Coles and Heimberg (2002) observed that with the exception of PD, there is little definitive evidence of an explicit memory bias for threat in any anxiety disorder. However, they note that while there are fewer studies of implicit memory, these do offer some indication of an implicit bias for threat.

Broadly speaking, the most evident resemblance between this and the previously reviewed research is a lack of consensus concerning memory (with the possible exception of PD). However, much information processing research in both clinical and non-clinical anxiety is affected by methodological problems that limit the validity of findings. One key concern is the appropriateness of the stimuli to the particular disorder under study. Coles and Heimberg (2002) observe that the most consistent evidence of memory bias has emerged from studies with stimuli that have high validity for the population in question. When examining information processing biases in anxiety, stimuli should 
ideally have high construct validity (i.e., the stimuli should clearly represent the constructs of interest, whether that is threat, positiveness, or neutrality). In addition, stimuli should have high specificity (i.e., should represent the particular type of threat that is salient to a particular type of anxiety). For example, findings of a significant memory bias have emerged from research on PD using words that represent panicspecific threat such as malls, crowds, palpitations, and dizziness (Becker et al., 1999). Similarly, memory biases in OCD have been found in research using real objects "contaminated" by a dirty tissue, thereby providing stimuli specific to OCD contamination fears (Radomsky \& Rachman, 1999). In these cases, memory biases were found when tasks involved stimuli with high validity and specificity for each disorder. In fact, Becker et al. (1999) suggested that the consistency of memory findings in PD may be due to the relative ease of providing stimuli that represent PD-specific threat (i.e., physical anxiety symptoms and unsafe places). It may be, therefore, that inconsistent findings in previous memory research are partly due to issues with the stimuli employed. GAD, which is characterized by worry about a wide range of topics, presents a particular challenge in terms of generating stimuli that are both valid and specific to the disorder.

\section{Summary of Information Processing Research}

The information processing research reviewed in the preceding pages can be summarized as follows. Research suggests that Intolerance of Uncertainty (IU) is associated with the selective processing of uncertain and threatening information. Specifically, high IU individuals show an attentional bias for threat and uncertainty; a tendency to make threatening interpretations of uncertain and negative information; and an enhanced memory for uncertainty. 
Given the few studies to date that have examined the role of IU in information processing, research with worriers and GAD patients was reviewed. Research suggests that GAD patients and high worriers have an attentional bias for threat, and make threatening interpretations of ambiguous information. However, evidence of a memory bias is mixed. More specifically, there is little evidence of an explicit memory bias in worry and GAD, but some evidence of an implicit memory bias.

As high trait anxiety is closely to related to worry and GAD, this research was also considered. High trait anxious individuals tend to show the same biases in attention and interpretation as high worriers and GAD patients; as in worry and GAD, memory findings were mixed. In high trait anxiety, however, there was slightly more support for explicit than implicit memory biases.

Finally, key findings from information processing research in other anxiety disorders were noted. Biases for disorder-specific threat have been found in each of the anxiety disorders. However, the strength of evidence for attention and interpretation biases varies considerably across disorders. With the exception of PD, there is little consistent evidence for explicit memory bias in the anxiety disorders, but some evidence of implicit biases.

\section{Critique of Research on Memory Bias in GAD and Worry}

The findings reviewed above have led some researchers to propose that anxiety is characterized by an attentional bias for threat, but not an explicit memory bias (Williams, Watts, MacLeod, \& Mathews, 1997; discussed below). However, other researchers suggest that inconsistent findings with regards to memory may be explained by the procedural variability across studies (Coles \& Heimberg, 2002). In fact, there are a 
number of methodological shortcomings in the existing research, which may well account for mixed results.

Validity and specificity of stimuli. Most studies of memory in GAD and worry have employed word stimuli. This is likely due to the effectiveness of words as stimuli in attention research, and their adaptability to information-processing tasks. However, the validity of the stimuli used in previous studies can be questioned on several grounds. For example, some studies have employed anxiety specialists (researchers or practitioners) to generate or select word stimuli (i.e., Becker et al., 1999; MacLeod \& McLaughlin, 1995; Mogg \& Mathews, 1990; Mogg et al., 1987). However, many researchers have selected stimuli solely on the basis of face validity. Even in the case of expert-selected stimuli, the content validity of the words to participants has rarely been evaluated. Consequently, it is difficult to know whether "threat", "neutral", and "positive" words adequately represented their intended constructs. Without this information, the comparison of responses to words from different categories is less meaningful. A related concern is that the concept of threat has been differently defined across studies. For example, threat has been defined in terms of physical threat, social threat, or even depression; in several studies, different concepts have been combined to form a single threat category (e.g., Friedman et al., 2000; MacLeod \& McLaughlin, 1995; Mathews et al., 1995; Otto et al., 1994). Similar mixtures have been made in categories of neutral and positive words. Acquiring participant ratings of the meaning of word stimuli could resolve many of these issues; however, this approach has rarely been used. Some researchers have obtained participant ratings of stimuli following the study, which allows for post-hoc verification of stimulus validity (i.e., Becker et al., 1999; Mathews, Mogg, et al., 1989). Ideally, 
however, stimuli would be pre-selected for research based on participant ratings. Only one study has used this approach, by selecting threatening, positive, and neutral words on the basis of participant ratings; the results showed an implicit memory bias for threat words (Coles et al., 2007). A limitation of this study, however, was that while each GAD patient was tested on their own idiosyncratic set of threat words, each yoked control participant was also tested on that patient's set of words. A greater bias might therefore be expected for the group tested on their own themes of concern (i.e., the GAD group). Nevertheless, using stimuli that have been rated and selected by participants has the potential to establish the validity of the stimuli for the population being studied.

Some studies have found a memory bias when stimuli were specifically tailored to the type of anxiety being studied. For example, memory biases have been found when disorder-specific words have been used to test individuals with Post-traumatic Stress Disorder (Kaspi, McNally, \& Amir, 1995; Vrana, Roodman, \& Beckham, 1995) and Panic Disorder (Becker et al., 1999; Otto et al, 1994). As previously noted, Becker et al. (1999) commented that findings of an explicit memory bias in Panic Disorder may be due to the ease of generating words specific to panic. Still other studies have used stimuli that may have better ecological validity than words. For example, memory bias has been found in studies using threatening facial expressions in Social Phobia (Lundh \& Öst, 1996), and contaminated objects in OCD (Radomsky \& Rachman, 1999). In all these cases, it appears that the specificity of the stimuli to the type of anxiety being studied aids in the detection of a memory bias.

Given the diversity of threat themes in GAD, and the idiosyncratic nature of worry, generating disorder-specific stimuli for GAD is a challenge. While some 
researchers suggest that word stimuli lack ecological validity (i.e., McNally, 1995), words have nevertheless been successfully used to elicit attention and interpretation biases in GAD and worry. In other anxiety disorders, when words are sufficiently specific, they also appear to be effective. Moreover, it is difficult to conceptualize a more ecologically valid set of stimuli for GAD. Whether words, pictures, or objects are considered, generating stimuli that would adequately represent threat and worry themes across high worriers would be extremely difficult. In fact, a word or passage of text may be more easily applied to personal concerns than an image. For example, words such as heart attack or loser, presented without any specific context, may naturally be processed in terms of personal associations to those words. Conversely, an image is likely to contain highly specific context and features (i.e., setting, gender and ethnicity of individuals depicted, etc.), which may prevent participants from relating the stimuli to their own experience. Furthermore, research suggests that threatening images are particularly aversive to worriers and GAD patients. If images are highly aversive to worriers, they may avoid focusing on threatening images, thereby interrupting any processing that might lead to enhanced memory (Friedman et al., 2000; see The role of cognitive avoidance, below, for further discussion). While not without limitations, therefore, word stimuli may at this time be a reasonable choice for testing memory biases in GAD and worry.

In order to address the diversity of threat and worry themes, many studies have included different types of threat words in their stimuli. This creates the risk that many words will not be relevant for a given participant. Given the small number of stimuli that typically make up a category of words, this could make biases extremely difficult to detect. For example, some high worriers may be sensitive to words denoting social threat 
(i.e., embarrassed, lonely), while others are not; similarly, words like heart attack may be relevant for some GAD patients, but are more likely to be relevant to individuals with Panic Disorder. Therefore, presenting GAD patients and high worriers with mixed types of threat stimuli may be an imperfect solution. Other researchers have used words representing worry themes that are typical for GAD patients (Becker et al., 1999; Coles et al., 2007). This approach has had mixed success, possibly due to the same issue of individual variability in threat and worry themes. One study to date has used a different approach, by using words that denote uncertainty, rather than threat; in this study, an explicit memory bias for uncertain words was found (Dugas, Hedayati, et al., 2005). As previously noted, IU is particularly high among worriers and GAD patients. In addition, although IU has been noted in other anxiety disorders, the weight of current evidence suggests that it is most strongly related to worry. Given the close relationship between $\mathrm{IU}$, worry, and GAD, it may be that uncertainty is the quality most aversive to high worriers and GAD patients. While a bias for threat may also be found in GAD because it is an anxiety disorder, biases may be clearer for stimuli related to its key cognitive vulnerability: intolerance of uncertainty. The use of stimuli that represent an underlying cognitive process, rather than specific worry themes, may overcome issues of variability in worry themes. While further research has yet to replicate the finding of a memory bias for uncertainty, the use of uncertain words as stimuli is an intriguing possibility.

Finally, if we intend to compare memory for different types of words, the words should be matched, or equivalent, on everything but the dimension of interest (i.e., threat value, uncertainty). For example, if one word in a set is distinctive compared to another (i.e., is highly imageable or unfamiliar), it may be remembered for that reason, and not 
because it represents a certain construct. For example, depending on the type of test and the familiarity of a given set of words, high or low frequency words may be better remembered (see Roediger \& McDermott, 1993, for a review). For this reason, most studies to date have matched word stimuli on familiarity, by controlling for the frequency of their occurrence in English (e.g., Carroll, Davies, \& Richman, 1971; Francis \& Kucera, 1982). As differences in length and part of speech can also make words more memorable, these features are also usually controlled. However, research shows that other properties can have a significant impact on processing and memory for words. Imageability, for example, refers to the ease with which a mental image can be formed of a word; concreteness refers to the degree to which a word relates to objects, persons, places, or things that can be experienced by the senses. Research shows that highly imageable and highly concrete words are more memorable than abstract words (Altarriba, Bauer, \& Benvenuto, 1999; Paivio, Yuille, \& Madigan, 1968). However, no study to date has controlled for the imageability or concreteness of stimuli. In fact, many studies have contrasted threat words with neutral words; most sets of neutral words have been both concrete and imageable (i.e., doorbell, chair). If the words used as a control condition are more concrete and imageable than other words, any bias for threat may well fail to be detected. In addition to imageability and concreteness, the emotionality of stimuli should be controlled. On one level, this implies that the emotional valence of words should be assessed, to ensure that positive words are significantly more positive, negative words more negative, and neutral words at a mid-point, on a continuum of emotionality. On another level, emotionality itself may be preferentially processed. Some researchers have questioned whether GAD patients preferentially process emotional information, rather 
than threat per se (Becker et al., 1999; Martin et al., 1991). Several studies of attention have actually found biases toward both positive and threatening stimuli in GAD patients (see Mogg \& Bradley, 2005, for a review). The inclusion of positive stimuli permits an exploration of whether biases concern threat (i.e., a bias toward threat words alone) or emotionality in general (a bias for both threat and positive words). While some studies to date have measured the valence of words, and included positive stimuli, many have not controlled for emotionality in this manner.

Encoding tasks and memory tests. In addition to stimulus variations, studies on memory bias in worry and GAD have differed widely in terms of procedure; this adds to the difficulty of drawing clear conclusions. The most evident divergence concerns the type of encoding tasks studies have used, including colour-naming, imagery, lexical decision making, and dichotic listening. One similarity, however, may be the prevalence of self-referent encoding tasks; fully half of the reviewed studies have used some form of self-referenced encoding. As previously noted, self-referenced tasks produce superior memory effects in non-clinical populations (Symons \& Johnson, 1997). Self-referenced tasks are thought to result in enhanced memory because they encourage deep, elaborative processing. According to the levels of processing theory (Craik \& Lockhart, 1972), encoding of information can occur on several levels: shallow processing, concerning sensory and physical information; deeper processing involving pattern recognition and matching; and deep processing, which involves semantic elaboration of the meaning of an item. According to this view, retention of information depends on how deeply an item is processed, with deeper processing resulting in better memory retention. In accordance with this view, research suggests that relating information to the self facilitates both the 
organization and the elaboration of material—in other words, deep processing — which results in enhanced memory (Klein \& Loftus, 1988; Symons \& Johnson, 1997). Selfreferenced encoding tasks have therefore seemed an obvious choice for studies of memory bias. As previously noted, however, self-referenced tasks do not consistently produce an advantage for threatening information among GAD patients and worriers, an apparently anomalous finding to which we will return. For the moment, however, it should be noted that half of the studies in this area employed highly elaborative, selfreferent encoding, while the remainder used tasks that varied considerably in the type and level of processing they required.

A review of this area of research should consider not only levels of processing, but the type of processing that tasks require. Research suggests that instructions given during encoding will determine the type of processing that will take place. For example, instructions to generate a word based on semantic cues (i.e., find the antonym for hot) will lead to conceptual processing, which involves the elaboration of semantic meaning. Conversely, instructions to focus on visual features (i.e., count the number of letters in hot) will lead to perceptual processing, which concerns surface information, such as visual or auditory features, without reference to meaning (Jacoby, 1983; Roediger, 1990). In accordance with these ideas, several studies have shown that under conditions of conceptual processing, memory for conceptual but not perceptual aspects of stimuli are found. Similarly, perceptual processing appears to enhance memory for perceptual but not conceptual aspects of a stimulus (Blaxton, 1989; Jacoby \& Dallas, 1981; Smith \& Branscombe, 1988). In fact, some research suggests that requiring participants to focus on perceptual features of a stimulus reduces their conceptual memory for that item; the 
reverse may also be true, with conceptual encoding reducing memory for perceptual features (Jacoby, 1983). These studies imply that task requirements determine what type of information will be encoded with regards to a stimulus; this then determines what is later available for retrieval. This view, termed the transfer-appropriate procedures approach, contrasts somewhat with levels of processing theory. Jacoby notes that deep (i.e., conceptual) processing does not necessarily create better memory for the stimulus, but rather determines what aspects of the stimulus will be remembered. In other words, conceptual processing enhances memory for conceptual aspects of a stimulus; similarly, perceptual processing does not necessarily imply shallow processing and poorer memory, but rather memory for perceptual features of a stimulus (Jacoby, 1983; see also Roediger, 1990). Of further note is that standard tests of explicit and implicit memory tend to measure different types of processing. For example, most explicit tests of memory involve the retrieval of semantic, elaborated information (i.e., free recall). In contrast, implicit tests often require retrieval based on partial perceptual information (i.e., degraded pictures, word stems). Therefore, tests of explicit memory might be better termed conceptually driven tests, and implicit tests perceptually driven; this is the source of some debate in the literature (see Roediger, 1990; Schacter, Chiu, \& Oschner, 1993, for overviews). For the purposes of the current discussion, however, it is sufficient to observe that a failure to match tasks and tests on processing requirements will increase the likelihood of non-significant findings. In fact, the concept of transfer appropriate procedures is based on the broader idea of transfer appropriate processing, the wellestablished finding that memory effects are greatest when the conditions at testing resemble those at encoding (Graf \& Ryan, 1990; Morris, Bransford, \& Franks, 1977). 
These principles together underscore the importance of matching study conditionsincluding type of processing - between encoding and testing.

In this light, memory research on GAD and high worry displays a dearth of transfer appropriateness. Of the twelve studies reviewed, only two were clearly transfer appropriate, with perceptual encoding tasks followed by perceptual implicit tests of memory; while one of these studies found a memory bias for threat, the other did not (MacLeod \& McLaughlin, 1995; Mathews et al., 1995). Two other studies are difficult to classify: one followed 'read only' encoding with an explicit free recall test (a bias was found: Friedman et al., 2000); in another, dichotic listening was followed by an explicit test of recognition (no bias was found: Mathews \& Macleod, 1986). Another study used a perceptual encoding task, with an explicit recognition test, which would be transfer "inappropriate"; in this study, no bias for threat was found (Mogg et al., 1989). All of the remaining seven studies used conceptual, primarily self-referenced encoding, which as previously noted involves a high degree of semantic elaboration. Further, all of these studies assessed explicit forms of memory, which would be considered transfer appropriate. However, four of these studies also assessed implicit memory; a significant bias for threat only emerged from two. Given that implicit tests were transfer inappropriate for these studies, the two non-significant findings are not surprising. What is surprising, however, is that any significant implicit memory bias could emerge from transfer inappropriate studies. However, a closer examination of the type of implicit test used-word stem completion — may explain this finding.

While word stems have been widely used as a test of implicit memory, research suggests that word stem completion is influenced by explicit memory. Imagine, for 
example, a participant who is asked to complete a set of word stems "with the first word that comes to mind", and in so doing notices that the stems correspond to words encountered during an earlier encoding task. In this case, the word stem completion becomes a form of explicit, cued recall. Studies suggest that participants do tend to become aware of the purpose of word stem tasks (i.e., Light \& Singh, 1987; Schacter, 1987). Moreover, research shows that word stem completions are significantly related to measures of explicit memory such as recall and recognition (Perruchet \& Baveux, 1989). In contrast, a task such as tachistoscopic word identification has been found to be unrelated to explicit memory, causing some researchers to deem it a purer measure of perceptual implicit memory (i.e., Perruchet \& Baveux, 1989; MacLeod \& McLaughlin, 1995; Tarsia, Power, \& Sanavio, 2003). It is notable that although both of the transfer appropriate studies mentioned above used perceptual encoding (an emotional Stroop task), only the study using a tachistoscopic word identification test found a significant memory bias for threat (MacLeod \& McLaughlin, 1995). The other study (Mathews et al., 1995) used a word stem completion test, which, given the above account, may explain the failure to find an implicit memory bias.

To summarize, there are a number of methodological problems with memory research on worry and GAD. For example, the stimuli employed in these studies have rarely been rated or selected by participants. This raises questions about the validity of stimuli, and whether they adequately represent their intended constructs for the population being studied. Furthermore, the construct of threat has been broadly or differently defined across studies, bringing the specificity of the stimuli for GAD and worry into question. Some recent research suggests that using uncertain stimuli-which 
represent a cognitive vulnerability specific to worry and GAD—may be a potential solution. Additional areas for improvement concern measuring and matching stimuli on aspects of emotionality, imageability, and concreteness, all of which affect processing and memory for words. Finally, studies in this area have varied widely in the encoding tasks they have employed. One trend has been the use of self-referent encoding, which produces superior memory effects in non-clinical populations, but does not appear to have this effect in GAD patients and worriers. Part of the reason for this may be the lack of distinction between conceptual and perceptual processing, and a failure to match tasks and tests on this dimension (i.e., a lack of transfer appropriate procedures). A related issue is that the word-stem completion test, which has often been used to measure implicit memory, appears to be influenced by explicit memory. Conversely, perceptual tests such as tachistoscopic word identification seem to more purely measure implicit memory. In fact, one of the few transfer appropriate studies that found a memory bias employed a tachistoscopic test. Together, these observations suggest that problems with stimuli, tasks and tests, and a lack of transfer appropriate processing, may explain many non-significant research outcomes.

\section{Theoretical Accounts of Existing Findings}

The Williams et al. (1997) model. The limitations described above may explain a lack of consistency across studies, and many mixed or non-significant research findings. However, they do not as easily explain why biases appear to be fairly consistent with regards to attention and interpretation, but rarely consistent on tests of memory. Some researchers have proposed that this pattern of findings actually represents the information-processing profile that is characteristic of anxiety. Williams and colleagues 
(1997) have suggested that different emotional states such as anxiety and depression are characterized by different patterns of cognitive processing, which do not operate in the same way across all stages of processing. They specifically propose that anxiety is primarily characterized by biases in attention and interpretation, but not in explicit memory. As the biological and social function of anxiety is to allow the individual to quickly cope with threat, attention and interpretation biases in anxiety would be adaptive. However, extensive elaboration of a threatening stimulus would not necessarily be adaptive, and might actually interfere with environmental vigilance for threat. For example, it may be useful for an individual to quickly recognize threat in the environment (there may be a bear in the trees!) but it might be less useful to elaborate on that stimulus (What species of bear is it? Is this their usual habitat? Are they vegetarian?). Williams et al. propose that it is this latter type of elaboration that would create a memory bias for threat stimuli. In their model, attention for threat is assumed, but without the type of elaboration that would lead to enhanced explicit memory.

More specifically, the Williams et al. (1997) model proposes that information from the environment is quickly evaluated on its threat value (high or low), through a perceptual, pre-attentive Affective Decision Mechanism. If the stimulus is assigned a high threat value, an automatic Resource Allocation Mechanism directs further processing toward threat. Subsequent cycles of information uptake are also informed by previous experiences with threatening stimuli (i.e., threat schemata). Through this allocation of attention to perceptual aspects of the threat, the stimulus becomes perceptually primed; that is to say, when partial perceptual cues are later presented, the stimulus will be more easily reconstructed. According to Williams and colleagues, this 
occurs through integrative processing, the automatic strengthening of the components of a mental representation. Integration is thought to make the stimulus more accessible when any aspect of it is later encountered (see also Graf \& Mandler, 1984). A presentation of partial visual cues is typical of perceptual implicit tests of memory (e.g., degraded pictures, tachistoscopic identification). The Williams et al. theory therefore predicts an implicit perceptual memory bias for threat stimuli.

However, the same stimulus will not necessarily be more retrievable in terms of explicit memory. Williams et al. (1997) contrast integration with elaboration, which is a form of strategic processing associating the stimulus to other mental representations. Elaboration is analogous to conceptual processing, and is thought to make stimuli more retrievable. Increased retrievability would be shown on tests of explicit memory such as recall or recognition. In the Williams et al. model, although attention is allocated toward threat, processing resources are not subsequently devoted to further elaboration of the threat stimuli. Therefore, in this model, an explicit conceptual memory bias for threat is not predicted.

Based on their model, Williams and colleagues (1997) propose that high trait anxious and anxiety-disordered individuals more readily identify stimuli as threatening, and more often direct attentional processing resources toward threat, without showing an explicit memory bias. Non-anxious individuals, in contrast, tend to direct attentional resources away from threat, unless stimuli are high in threat value. It should be emphasized that the assignment of threat value (through the Affective Decision Mechanism) is thought to occur automatically, at a pre-attentive level. Similarly, the Resource Allocation Mechanism is thought to operate automatically. Furthermore, 
Williams et al. propose a different allocation of processing resources in depression as compared to anxiety. They contend that depression is characterized by increased elaborative processing of negative stimuli, but not increased integration. Therefore, the model predicts enhanced conceptual, explicit memory for negative information in depression, without an attention bias. Furthermore, the researchers note that different anxiety disorders may be characterized by differing degrees of elaborative processing, such that in some anxiety disorders (i.e., phobias) there may be a stronger tendency to direct resources away from further elaboration. Finally, Williams et al. acknowledge that both automatic and strategic processes are likely to contribute to information processing; they only propose that certain modes will predominate in anxiety, and others in depression.

The Williams et al. model might account for many current research findings in trait anxiety and GAD. They note that, in accord with their predictions, attentional biases are consistently found among anxious individuals, while explicit memory biases are rare; furthermore, there is some evidence of implicit memory biases. Recall that in the literature review, six of the GAD studies used conceptual self-referenced encoding, and all of these assessed explicit memory, in accord with transfer-appropriate procedures. However, only one of these studies found an explicit memory bias, and this study warned participants their memory would be tested (Mogg \& Mathews, 1990). Therefore, there does appear to be some research support for the idea that an explicit memory bias does not characterize GAD. In addition, research supports the proposal that attentional biases can be observed at the pre-attentive level (e.g., Bradley, Mogg, Millar, et al., 1995; Mogg et al., 1995; Mogg et al., 1993). Finally, although attentional biases are rarely found in 
depression, an explicit memory bias for negative information is often found (see Mathews \& MacLeod, 2005, for a review).

Nevertheless, Williams and colleagues (1997) acknowledge that not all findings fit their model. They note several failures to find an implicit memory bias, and attribute these in part to some of the methodological issues already reviewed, including a lack of transfer-appropriate procedures. In some studies, however, conceptual tasks actually produced evidence of an implicit memory bias. In most of these studies, however, word stems were used to test implicit memory, and, as already discussed, word stems possibly provide a largely explicit measure of memory. Williams and colleagues also recognize that while few in number, studies that have found an explicit memory bias are problematic for their model. First, they note that if participants are actually required by the task to engage in elaborative processing of the stimuli, an explicit bias may result (e.g., Mogg \& Mathews, 1990). Alternatively, they propose that depression, which often co-occurs with anxiety, may account for some instances of explicit memory bias. While few studies have measured or controlled for depression, in some studies depression has indeed been found to contribute to explicit memory for threatening or negative information (e.g., Becker et al., 1999; Bradley, Mogg, \& Williams, 1995; Mogg et al., 1989).

In summary, the Williams et al. (1997) model predicts that anxious individuals will show both attention and interpretation biases for threat. This leads to enhanced accessibility of threat stimuli: in other words, an implicit perceptual memory bias for threat. However, because elaborative processing of threat does not occur, there should be no explicit conceptual memory bias for threat. Essentially, this model predicts a pattern 
of bias for threat in attention and interpretation, but not in explicit memory; however, an implicit memory bias for perceptual aspects of threat are predicted. While this model is consistent with much of the existing data, there are discrepancies, some of which may be attributable to methodological shortcomings or to the influence of depression on memory.

Cognitive avoidance. While much research has focused on a bias toward threat, anxiety and the anxiety disorders are also characterized by avoidance of threat. For example, anxiety disorders are associated with avoidance of threatening objects or situations (e.g., spiders in Spider Phobia; social interactions in Social Phobia). Anxiety disorders are also associated with avoidance of threatening cognitions (e.g., intrusive thoughts in Obsessive-Compulsive Disorder; traumatic images in Post-Traumatic Stress Disorder), in other words, cognitive avoidance. It can be imagined that the tendency to avoid upsetting cognitive material would affect memory for threatening information. For example, an anxious individual may quickly notice and attend to a threatening stimulus, but then engage in cognitive avoidance to reduce their unpleasant emotional response. In this scenario, less processing of the threatening information would occur, resulting in weaker memory. The finding that GAD patients do not consistently show enhanced memory for self-referenced material may be related to cognitive avoidance. Friedman and colleagues (2000) suggest that a task relating threatening information to the self may be too anxiety-provoking for GAD patients, who may engage in cognitive avoidance to reduce their anxiety; they note that this may be particularly true for self-referent imagery. In this case, a memory bias would be less likely to result.

In fact, research indicates that cognitive avoidance is closely related to both GAD and worry. As already noted, research has found attention biases toward threat among 
high trait anxious individuals and GAD patients. However, some studies show that when longer response latencies are examined, attention shifts away from threat (see reviews by Cisler, Bacon, \& Williams, 2009; Cisler \& Koster, 2010). This suggests initial attention toward threat, followed by a shift of attention away from threat, a pattern that would be consistent with cognitive avoidance. In addition, research has shown elevated levels of cognitive avoidance in both GAD patients (Ladouceur et al., 1999) and high worriers (Dugas, Marchand, et al., 2005). While different types of cognitive avoidance may be due to automatic or effortful processes (i.e., Dugas \& Robichaud, 2007), the measurement of automatic cognitive avoidance presents obvious difficulties. Therefore, much existing research on cognitive avoidance concerns relatively conscious and effortful processes. For example, research shows that worry and GAD are associated with several forms of cognitive avoidance, such as substituting worries with positive thoughts; distraction from worry; avoiding cues that may trigger worry; and suppression of worrisome thoughts (Dugas, Marchand, et al., 2005; Dugas \& Robichaud, 2007). Consequently, several models of GAD include cognitive avoidance as a process that contributes to and maintains worry (e.g., Borkovec et al., 1998; Dugas, Marchand, et al., 2005 ).

Some researchers have suggested that worry is itself a form of cognitive avoidance. For example, research shows that threatening mental images are more emotionally arousing than the abstract, verbal-linguistic thought characteristic of worry (Vrana, Cuthbert, \& Lang, 1986). Furthermore, research suggests that worrying can actually reduce both mental imagery and physiological reactions to threat (Borkovec, Lyonfields, Wiser, \& Deihl, 1993). In their cognitive avoidance theory of worry, Borkovec and colleagues therefore suggest that worrying permits avoidance of the 
unpleasant anxious arousal associated with threatening mental images (Borkovec, Alcaine, \& Behar, 2004). This is consistent with the finding that high worriers report that worrying allows them to avoid thinking about even more upsetting topics (Borkovec \& Roemer, 1995). According to this view, although worry is associated with tension and anxiety, it may prevent the more intense anxiety and arousal associated with frightening images (i.e., the image of one's child in a car accident). Borkovec and colleagues also propose that worry prevents full emotional processing of fear. According to Foa and Kozak's (1986) theory, activation of all aspects of fear (cognitive, behavioural, affective, physiological) is necessary for corrective emotional processing to occur. Without full emotional activation, fears are not fully emotionally processed, and are thereby maintained. Consequently, the cognitive avoidance theory of worry suggests that worry actually maintains fears (Borkovec et al., 2004). Furthermore, every time that worry reduces unpleasant emotional and physiological arousal, it may be negatively reinforced. This negative reinforcement would perpetuate and maintain the tendency to worry. In fact, some research indicates that worrying about a stressful situation reduces short-term anxiety, but generates more intrusive thoughts in the long term (Butler, Wells, \& Dewick, 1995; Stokes \& Hirsch, 2010; Wells \& Papageorgiou, 1995). Taken together, this research suggests that worry may be a form of cognitive avoidance that interferes with the processing of threatening information by anxious individuals. As with other forms of cognitive avoidance, worry would be expected to direct processing — particularly elaborative processing — away from threat, thereby reducing memory for it.

Some parallels can be drawn between the Williams et al. (1997) model and theories of cognitive avoidance. In these theories, attention is initially directed toward 
threat, but an intervening process directs further processing away from threat, thereby inhibiting the type of elaboration that would result in a memory bias. Williams and colleagues, however, do not conceptualize this re-direction of processing resources as effortful, and they disagree with some of the assumptions made by Borkovec's cognitive avoidance theory of worry. In addition, the Williams model is the only one to specifically predict an implicit perceptual memory bias for threat. However, some degree of implicit memory bias might be expected in the cognitive avoidance theory, due to the initial attention allocated toward threat. Despite their differences, therefore, the theories reviewed above converge in their prediction of initial attentional biases toward threat, without a subsequent explicit memory bias.

\section{General goals of the current research}

As is evident from the preceding review, many studies of GAD patients have failed to find a memory bias for threat. However, an explicit memory bias for threat has emerged from a few studies of GAD and high trait anxious individuals. In addition, some other anxiety disorders such as Panic Disorder have demonstrated explicit memory biases for threat. Therefore, one goal of the current study is to re-examine the question of explicit memory bias for threat. A review of the literature also shows that while findings are mixed, several studies indicate that worriers and GAD patients have an implicit memory bias for threat. The Williams et al. (1997) theory, which predicts an implicit but not an explicit bias for threat in anxiety, will be used to guide an investigation of implicit memory. Accordingly, the second goal of this study is to explore implicit perceptual memory bias for threat. 
A central goal of the current research is to evaluate the contribution of IU to memory biases. Most previous research has focused on the presence or absence of memory bias in GAD or trait anxiety; the impact of a cognitive vulnerability such as IU has yet to be examined. Given that IU is not only highly related to worry and GAD, but is a dispositional vulnerability for worry and GAD, levels of IU may affect memory biases for threat. It could be hypothesized that worriers higher in IU may show a stronger memory bias for threat than those lower in IU. Given the close relationship between IU and worry, worry is also expected to be related to memory biases for threat. However, IU - a cognitive disposition — is expected to be a better predictor of memory bias than worry - a symptom or manifestation of IU. While one study to date suggests a connection between IU and memory bias, this is a new area of exploration. A key objective of this research is therefore to assess the contribution of IU to memory biases for threat.

Intolerance of uncertainty will also be examined on another level in this study: that of the stimuli. As already noted, IU as a dispositional characteristic is expected to make a contribution to memory biases for threat. However, because uncertainty is a source of distress for individuals high in IU, we might expect that stimuli denoting uncertainty will be preferentially processed. This does seem to be the case in the few studies that have used uncertain stimuli to date. It can be supposed that threatening stimuli would be threatening to most individuals, and more threatening to anxious individuals. Uncertain stimuli, however, would be most threatening to individuals who are highly intolerant of uncertainty. The use of uncertain stimuli may therefore provide a highly specific means of examining memory biases in worry. Accordingly, another goal of this study is to assess memory bias for uncertain as well as threatening stimuli. 
Contemporary research and theory suggest that other processes may contribute to memory outcomes in worry and GAD. Cognitive avoidance, for example, may lead to a lack of elaborative processing, and hence a lack of memory bias. Consequently, different forms of cognitive avoidance will be measured in this study. Another potential influence that will be considered is depression. The few findings of an explicit memory bias in anxiety may in fact be due to co-occurring depression, given that depression is characterized by an explicit memory bias. For this reason, moderate to high levels of depression will be an exclusionary criterion in this research, and symptoms of depression will be measured and analyzed. Other factors known to affect performance on information processing tasks will also be assessed, including state anxiety, irritability, fatigue, attentional control, and social desirability.

An over-arching goal of this research is to address the methodological problems found in previous studies. In order to establish the validity of the stimuli, for example, stimulus words will be pilot tested before hypotheses concerning memory bias are explored. Specifically, participants will be asked to rate the properties of candidate words, and only those that correspond to the constructs of interest, and can be matched on other dimensions such as familiarity, will be used in the memory studies. In the studies concerning explicit and implicit memory, transfer-appropriate procedures will be strictly observed. Specifically, explicit conceptual processing will be matched with explicit memory tests, and implicit perceptual processing will be matched with implicit tests. To this end, tachistoscopic word identification will be used to assess perceptual implicit memory, as it has been deemed a relatively pure measure. 
In summary, the goal of the current research is to explore the relationship between IU, and explicit and implicit memory biases for threat and uncertainty. The potential influence of factors such as worry, cognitive avoidance, and depression will also be considered. To achieve these ends, a participant-piloted set of stimuli will be created, including stimuli denoting uncertainty. Throughout these investigations, procedural issues will be addressed, with a particular focus on the maintenance of transferappropriate procedures.

\section{Study goals and hypotheses}

In Study 1, pilot testing and selection of word stimuli for the research will take place. Candidate stimuli will first be rated by a group of participants on five dimensions: familiarity, uncertainty, imageability, concreteness, and valence. Word ratings be analyzed, and stimuli will be selected based on their distinctness with regards to constructs of uncertainty, positive valence, threat valence (social or physical), and neutral valence. Stimuli will then be matched on the remaining dimensions of familiarity, imageability, and concreteness, as well as length and part of speech. The result will be a matched set of stimulus words categorized as neutral, uncertain, socially threatening, physically threatening, and positive. Words representing uncertainty will be included in order to provide stimuli specific to worry and GAD. Both physical and social threat words will be used in order to explore any differences in processing for these different types of threat. Furthermore, positive words will be included so that any biases related to the emotionality of the stimuli can be evaluated. This method of piloting, statistical analysis, and matching is expected to produce a set of stimulus words with high validity 
and specificity. This procedure should rectify some of the problems with previously used stimuli, which may have prevented memory biases from being identified.

Study 2 will explore the relationship between IU and explicit memory bias for threat and uncertainty. While existing research does not offer strong support for an explicit memory bias for threat among worriers, this may be due to the limited validity and specificity of the stimuli that have been used. Therefore, using the stimuli created in Study 1, an explicit encoding task will be used to promote incidental learning, and explicit memory for the stimuli will be tested. Given the good validity and specificity of the stimuli that will be used in this study, it is hypothesized that IU will be a significant predictor of explicit memory bias for threatening and uncertain words. Exploratory analyses will be assess the influence of factors such as worry, cognitive avoidance, and depression.

Study 3 will examine the relationship between IU and perceptual, implicit memory bias for threat and uncertainty. Specifically, participants will engage in an incidental learning task that promotes perceptual processing. They will then complete a perceptual test (tachistoscopic word identification) in order to assess their implicit perceptual memory for the stimuli. Transfer-appropriate procedures will be strictly observed, with both task and test requiring perceptual processing. Based on the predictions of Williams et al. (1997), and the findings of previous studies, IU is expected to be significantly related to an implicit perceptual memory bias for threatening and uncertain words. A free recall test will be administered to explore any inadvertent effects of explicit memory; however, as outlined in the Williams et al. model, no explicit memory bias is predicted. As in Study 2, the contributions of worry, cognitive avoidance, 
and depression will be assessed. In Study 3, the impact of vocabulary, personal relevance of stimulus words, and attentional control will also be explored. 


\section{STUDY 1}

\section{Method}

\section{Participants}

Participants for this study were recruited through Concordia University's Psychology Department Participant Pool, and received one bonus course credit for participating (one credit $=$ a bonus $1 \%$ of their final grade). This method of recruitment and compensation was approved by Concordia University's Human Research Ethics Committee. Participants were eligible for the study if they were fluent in English, and between 18-65 years of age.

Fifty participants completed the study and received one course credit for their participation. The sample included 40 women and 10 men, aged 18 to 40, with a mean age of $23(S D=4.22)$. Seventy-two percent of the sample were White, $12 \%$ were Asian, 8\% Multi-racial, 4\% Black, 2\% First Nations, and 2\% Middle Eastern. All participants in this sample were students, of whom 74\% were Psychology majors. Seventy percent of the sample reported that English was their first language; 20\% reported their first language as "Other", and 10\% cited French as their first language. All participants reported being fluent in English.

\section{Materials}

Generation of words for pilot testing. Initially, the Medical Research Council (MRC) Psycholinguistic Database (http://www.psy.uwa.edu.au/mrcdatabase/uwa_mrc.htm) was consulted in order to obtain stimulus words for this study. While extensive, this database included few words denoting uncertainty. In addition, the MRC database does not include ratings on 
dimensions of uncertainty and valence, which are key for this study. For these reasons, it was decided that a new set of words would be selected and pilot tested for this study.

In order to generate a large pool of words for pilot testing, the anxiety information processing literature was searched for previously used sets of stimulus words (i.e., Dugas, Hedayati, et al., 2005; Matthews \& Macleod, 1985; Macleod et al., 1986; Manguno-Mire, Constans, \& Geer, 2005). Words that could be categorized as either positive, uncertain, or threatening were selected; for threat words, a distinction was made between words that were socially versus physically threatening. A small pilot test $(N=5)$ was conducted, with participants rating a pool of threat words on the following scale: purely socially threatening; mostly socially threatening; equally socially and physically threatening; mostly physically threatening; purely physically threatening. Words that were rated as equally socially and physically threatening were excluded, and words rated as either primarily social or primarily physical were retained.

Multiple synonyms for the literature-derived words were then obtained using various sources (i.e., Microsoft Word ${ }^{\circledR}$ (Microsoft Corporation) Thesaurus; Roget's College Thesaurus, Revised, 2000; Thesaurus.com synonym generator: http://thesaurus.com). The first author and another graduate student then reviewed the pool of words, and each independently selected pilot words that they felt best represented each of the categories. The raters then compared their lists of pilot words: words both had rejected were excluded; words both had selected were retained; and a consensus was reached about the remaining words. This process resulted in 40 positive, 40 social threat, 40 physical threat, and 40 uncertain words for pilot testing. 
Neutral words were generated last in order to match them to the other pilot words on length, part of speech, and familiarity. Each of the 160 pilot words was entered into the MRC Psycholinguistic Database, which was used to generate words that matched each pilot word on length and part of speech, and had a moderate familiarity rating (according to the MRC rating system, between 400-700). A research assistant then selected the three words she judged most neutral for each pilot word. The graduate raters then independently chose two neutral words for each pilot word. As with the other pilot words, the word lists were then compared and consensus was reached; this resulted in a set of 86 neutral words for pilot testing (a larger number of neutral words was required for use in practice trials).

The procedure described above resulted in a total of 246 words for pilot testing: 40 positive, 40 social threat, 40 physical threat, 40 uncertain, and 86 neutral words. Given that each of these 246 words would be rated five times (i.e., on five dimensions), it was predicted that accuracy of word ratings would suffer if participants were asked to rate the entire set of words. Therefore, the pilot words were randomly divided into two sets. Set 1 was made up of 123 words: 20 positive, 20 social threat, 20 physical threat, 20 uncertain, and 43 neutral words. Set 2 likewise included 123 words, with the same proportion of each word type. No word appeared in both sets (see Appendix A for Set 1 words).

Set 1 words were then mixed in randomized order, and inserted into a questionnaire asking participants to rate each word on its concreteness (questionnaires are described in detail below). Set 1 words were then inserted into four other questionnaires asking participants to rate the familiarity, imageability, uncertainty, and valence of each word. The same procedure was then followed for Set 2 words (see Appendix B). 
The five questionnaires containing Set 1 pilot words were placed in random order, with the constraint that familiarity was always the first questionnaire, as re-rating words could influence familiarity ratings. The same procedure was then followed for questionnaires containing the Set 2 pilot words. As explained below, Set 1 and Set 2 packages were later completed by separate groups of participants.

\section{Measures}

Concreteness questionnaire. This questionnaire was based on definitions of concreteness used in previous research (i.e., the MRC Psycholinguistic Database, http://www.psy.uwa.edu.au/mrcdatabase/uwa_mrc.htm, and Spreen \& Schulz, 1966). Instructions to participants were: "Words differ in the extent to which they refer to concrete objects, persons, places or things that can be seen, heard, felt, smelled, or tasted, as contrasted with abstract concepts that cannot be experienced by our senses. In this questionnaire you will be asked to rate a list of words with respect to their "concreteness" in terms of sensory experience. Any word that refers to objects, materials, or persons, should be given a high concreteness rating (....) Any word that refers to an abstract concept that cannot be experienced by the senses should be given a low concreteness rating (....).” In previous research, concreteness ratings were made on a 7-point Likert scale; this format was therefore retained, with each word rated from $1=$ least concrete, to 7 = most concrete (see Appendix C).

Familiarity questionnaire. This questionnaire was designed to assess how often participants had come into contact with the words. They were asked to rate each pilot word on how often they had seen, heard, or used the word in speech or writing, on a 5point Likert scale that ranged from 1 = never: you have never seen or heard or used the 
word in your life, to $5=$ very often: you have seen or heard or used the word nearly every day of your life. As this questionnaire concerned familiarity and not vocabulary, participants were asked to make a rating even if they were unsure of the word's meaning (see Appendix D).

Imageability questionnaire. The purpose of this questionnaire was to assess the ease with which a mental image can be formed of a word. The instructions are based on previous research ( i.e., MRC Psycholinguistic Database; Gilhooly \& Logie, 1980):

"Words differ in their capacity to arouse mental images of things or events. Some words arouse a sensory experience, such as a mental picture or sound, very quickly and easily, whereas other words may do so only with difficulty (i.e., after a long delay) or not at all. In this questionnaire you will be asked to rate a list of words on the ease or difficulty with which they arouse mental images. Any word that in your opinion arouses a mental image (i.e., a mental picture, or sound, or other sensory experience) very quickly and easily should be given a high imagery rating (....) Any word that arouses a mental image with difficulty or not at all should be given a low imagery rating (....).” The original 7-point Likert rating was retained, with words rated from $1=$ difficult to form a mental image, to 7 = easy to form a mental image (see Appendix E).

Uncertainty questionnaire. This measure was created in order to assess the extent to which words represented uncertainty. Preliminary piloting of this questionnaire and feedback from participants indicated that simple instructions were easier to follow when rating uncertainty. Therefore, participants were asked How much do each of the following words refer to uncertainty? and rated each word on a 5-point Likert scale 
ranging from 1 = does not refer to uncertainty, to $5=$ refers perfectly to uncertainty.(see Appendix F).

Valence questionnaire. The purpose of this questionnaire was to obtain ratings of the positive or negative qualities of each word, i.e., the word's valence. Participants were asked, How much of a negative or positive connotation do the following words have? Each of the words was rated on a 5-point Likert scale with the following anchors: $1=$ very negative; 2 = a little negative; $3=$ neutral (neither negative nor positive); $4=$ a little positive; 5 = very positive (see Appendix G).

A General Information Sheet was created to obtain demographic information about participants, specifically: age, sex, education, student status, field of study, first language, English language fluency, race/ethnicity (see Appendix H).

\section{Procedure}

Rating of words. Participants were tested in groups, in a classroom setting. Participants were informed that the purpose of the study was rate words on dimensions of concreteness, imageability, familiarity, uncertainty, and positive/negative characteristics (valence). Participants read and signed consent forms describing the study and conditions of informed consent (see Appendix I). Participants were randomly given either a Set 1 package, or a Set 2 package; these took approximately one hour to complete.

Debriefing. When word ratings were completed, participants were given a written Debriefing sheet (see Appendix J) concerning the purpose of the study, and how their ratings would be used to select stimulus words for use in future research. Finally, they were compensated for their participation with 1 bonus course credit. 


\section{Results}

\section{Data Screening}

Ratings of the pilot words were independently entered by two individuals (i.e., double-entered). The two entries were then compared, and discrepancies were corrected in order to ensure accuracy.

\section{Participant Characteristics}

A series of Chi-square analyses showed no significant differences between Set 1 and Set 2 participants in sex $\chi^{2}(1)=.50, p=.48$; race/ethnicity $\chi^{2}(5)=6.44, p=.27$; education level $\chi^{2}(3)=.75, p=.86$; or first language $\chi^{2}(2)=.63, p=.73$ (note that all participants reported being fluent in English). In addition, an independent samples $t$-test showed no significant age differences between Set $1(M=22.40, S E=.60)$ and Set 2 participants $(M=23.38, S E=1.07), t(47)=-.81, p=.13$

\section{Stimulus Word Selection}

The goal of stimulus word selection was to obtain sets of words that would be conceptually specific to their own category, while being equivalent to words in other categories on concreteness, familiarity, and imageability. To this end, a mean score was calculated for each of the 246 pilot words on each of the dimensions of concreteness, familiarity, imageability, uncertainty, and valence.

The set of uncertain words was selected first, as the goal was to match other word sets to the uncertain words. Uncertain words were placed in ranked order, from least to most concrete. Four more ranked lists of the uncertain words were then made based on familiarity, imageability, uncertainty, and valence. Uncertain words with extreme values on any of these dimensions were eliminated. The following criteria were then used to 
select the uncertain stimulus words: 1) high uncertainty; 2) moderate to high familiarity; 3) neutral valence; 4) high concreteness and high imageability (because uncertain words are low on these dimensions, the highest possible were chosen). As with pilot word generation, the first author was aided by lab colleagues in this task.

The other stimulus word sets were selected in a similar way. First, ranked lists of words in each category were generated, and words with extreme scores eliminated. Each set of words was then selected with reference to the primary characteristic of its category; as far as possible, words were then chosen that matched the uncertain words on familiarity, concreteness, and imageability. For example, the positive words selected were the highest on valence, moderate on familiarity, and low on uncertainty, concreteness, and imageability. Threat words were lowest on valence, moderate on familiarity, and low in uncertainty, concreteness, and imageability. Neutral words fell in the mid-range on valence, moderate in familiarity, and low on all other dimensions. In addition to these criteria, each of the words from the other categories was matched in length $( \pm 1$ letter) and part of speech to one of the uncertain words.

This procedure resulted in a set of 64 matched stimulus words, comprised of 16 neutral words, 16 uncertain words, 16 threat words ( 8 social, 8 physical), and 16 positive words (see Table 1). Equal numbers of social and physical threat words were subsumed in the category of threat words to simplify the research design, while providing the option of later analyzing these types of words separately. As previously noted, additional neutral words were selected for use in practice trials (i.e., for Study 3). 


\section{Properties of Stimulus Words}

The final set of stimulus words was analyzed to compare the concreteness, familiarity, imageability, uncertainty, and valence of each category of words. As the words had been administered in 2 packages (Set 1 and Set 2), and these made up two different sets of words, rated by different groups of participants, analyses were conducted separately for Set 1 and Set 2 (note: Set 1 and Set 2 words were combined to produce the final stimulus sets; the division of words into Set 1 and 2 was solely for the purpose of statistical analysis). For these analyses, each word was treated as a case, having a mean rating on each of the 5 dimensions (concreteness, familiarity, imageability, uncertainty, and valence). Because the five dimensions are qualitatively different, and moreover rated on different scales (i.e., 7-point scales for concreteness and imageability; 5-point scales for the other dimensions), each dimension was used as the outcome in a separate analysis. A series of one-way ANOVAs were conducted, with word category (neutral, uncertain, social threat, physical threat, positive) as the grouping variable, and ratings on one of the dimensions as the outcome. Post-hoc pairwise comparisons were then used to compare the word categories on the outcome dimension. Due to heterogeneity of variance, Welch's $F$ and Games-Howell pairwise comparisons were used. 
Table 1

Study 1: Matched Stimulus Set of Neutral, Uncertain, Social Threat, Physical Threat, and Positive Words

\begin{tabular}{|c|c|c|c|}
\hline Uncertain $^{a}$ & Threat $^{b}$ & Positive & Neutral \\
\hline vague & hated & great & rural \\
\hline maybe & rude & good & every \\
\hline chance & virus & worthy & entire \\
\hline tricky & mocked & adored & modern \\
\hline random & lethal & caring & actual \\
\hline varying & ignored & admired & literal \\
\hline puzzling & mutilated & talented & exterior \\
\hline doubtful & feverish & generous & apparent \\
\hline debatable & inferior & pleasant & customary \\
\hline irregular & ridiculed & optimistic & methodical \\
\hline imprecise & epidemic & wonderful & reciprocal \\
\hline uncertain & unsanitary & unselfish & unlisted \\
\hline ambiguous & sickness & confident & universal \\
\hline hesitation & disapproval & integrity & ingredient \\
\hline mysterious & incompetent & delightful & theoretical \\
\hline questionable & contaminated & enthusiastic & geographical \\
\hline
\end{tabular}

${ }^{a}$ Each uncertain word is matched to the other words in that row (i.e., vague is matched to hated, great, and rural).

${ }^{\mathrm{b}}$ Social threat words are italicized. 
While the goal was to achieve equivalent concreteness across categories, differences on this dimension emerged in Set 1 , Welch's $F(4,12.81)=54.27, p<.05$. Specifically, comparisons showed that in Set 1, uncertain words were significantly less concrete than social threat, physical threat, and positive words; furthermore, physical threat words were significantly more concrete than all other word types. Differences in concreteness were also found in Set 2 , Welch's $F(4,5.69)=10.11, p<.05$; in this case, however, uncertain words were only significantly less concrete than social threat and positive words (see Figures $1 \& 2$ ).

As expected, familiarity was equivalent across all word types. The results showed no significant differences in familiarity for any of the word categories in Set 1, Welch's $F(4,14.38)=1.95, p=.16$, or in Set 2, Welch's $F(4,3.86)=.79, p=.59$ (see Figures 1 \&2).

Results also indicated some disparity in imageability in Set 1 , Welch's $F(4,14.88)$ $=23.53, p<.05$. Pairwise comparisons showed that uncertain words were significantly less imageable than social threat, physical threat, and positive words. In addition, physical threat words were significantly more imageable than all other word types, except neutral. Differences were also found in Set 2, Welch's $F(4,5.68)=12.62, p<.05$; in this set, however, positive words alone were more imageable than social threat words (see Figures 1 \&2). 
Figure 1

Study 1: Pairwise Comparisons Between Word Stimuli on Dimensions of Concreteness, Familiarity, Imageability, Uncertainty, and Valence

\section{Set 1 Words}

\begin{tabular}{|c|c|c|c|c|c|}
\hline & $\begin{array}{l}\text { a - Neutral } \\
\text { Words }\end{array}$ & $\begin{array}{l}\text { b - Uncertain } \\
\text { Words }\end{array}$ & $\begin{array}{c}\text { c- Social Threat } \\
\text { Words }\end{array}$ & $\begin{array}{c}\text { d - Physical Threat } \\
\text { Words }\end{array}$ & $\begin{array}{l}\text { e - Positive } \\
\text { Words }\end{array}$ \\
\hline $\begin{array}{c}\text { Mean } \\
\text { Concreteness }\end{array}$ & $\mathbf{2 . 6 1}^{\mathrm{d}}$ & $\begin{array}{l}\mathbf{1 . 7 9} \\
.09\end{array}$ & $\begin{array}{c}\mathbf{2 . 8 9}^{\mathrm{bd}} \\
.43\end{array}$ & $\begin{array}{c}\mathbf{4 . 4 3} \\
.53\end{array}$ & $\begin{array}{c}\mathbf{2 . 8 6}^{\mathrm{bd}} \\
.31\end{array}$ \\
\hline $\begin{array}{c}\text { Mean } \\
\text { Familiarity }\end{array}$ & $\begin{array}{c}3.75 \\
.70\end{array}$ & $\begin{array}{c}\mathbf{3 . 8 8} \\
.51\end{array}$ & $\begin{array}{c}3.74 \\
.38\end{array}$ & $\begin{array}{c}3.27 \\
.50\end{array}$ & $\begin{array}{c}\mathbf{4 . 0 3} \\
.45\end{array}$ \\
\hline $\begin{array}{c}\text { Mean } \\
\text { Imageability }\end{array}$ & $\begin{array}{c}\mathbf{3 . 4 0} \\
1.64\end{array}$ & $\begin{array}{c}\mathbf{2 . 6 3} \\
.45 \\
\end{array}$ & $\begin{array}{c}\mathbf{3 . 8 0}^{\text {bd }} \\
.46\end{array}$ & $\begin{array}{c}\mathbf{5 . 3 0}^{\text {bce }} \\
.45\end{array}$ & $\begin{array}{c}\mathbf{4 . 0 3}^{\text {bd }} \\
.70\end{array}$ \\
\hline $\begin{array}{c}\text { Mean } \\
\text { Uncertainty }\end{array}$ & $\begin{array}{c}\mathbf{1 . 3 9}^{\mathrm{b}} \\
.29\end{array}$ & $\begin{array}{c}3.87^{\mathrm{ac}} \\
.53\end{array}$ & $\begin{array}{c}\mathbf{1 . 4 8 ^ { \text { be } }} \\
.16\end{array}$ & $\begin{array}{l}1.67^{\text {be }} \\
.20\end{array}$ & $\begin{array}{l}1.17^{\mathrm{bcd}} \\
.10\end{array}$ \\
\hline $\begin{array}{c}\text { Mean } \\
\text { Valence }\end{array}$ & $\begin{array}{l}3.16^{\text {cde }} \\
.24\end{array}$ & $\begin{array}{l}\mathbf{2 . 4 9 ^ { \text { cde } }} \\
.53\end{array}$ & $\begin{array}{l}1.50^{\text {abe }} \\
.23\end{array}$ & $\begin{array}{l}1.44^{\text {abe }} \\
.25\end{array}$ & $\begin{array}{c}4.66^{\mathrm{abcd}} \\
.15\end{array}$ \\
\hline
\end{tabular}

Note. For each word type, the mean rating for each dimension is shown in bold font, with the standard deviation in plain font beneath. Superscripts beside each mean indicate the other word types that significantly differ from that word type at the $p<.05$ level: ${ }^{a}$ neutral words, ${ }^{b}$ uncertain words, ${ }^{c}$ social threat words, ${ }^{\mathrm{d}}$ physical threat words, ${ }^{\mathrm{e}}$ positive words. A lack of superscript indicates no significant difference between that word type and other types. Due to heterogeneity of variance, Games-Howell pairwise comparisons were used. 
Figure 2

Study 1: Pairwise Comparisons Between Word Stimuli on Dimensions of Concreteness, Familiarity, Imageability, Uncertainty, and Valence

\section{Set 2 Words}

\begin{tabular}{|c|c|c|c|c|c|}
\hline & $\begin{array}{c}\text { a - Neutral } \\
\text { Words }\end{array}$ & $\begin{array}{l}\text { b - Uncertain } \\
\text { Words }\end{array}$ & $\begin{array}{c}\text { c - Social Threat } \\
\text { Words }\end{array}$ & $\begin{array}{c}\text { d - Physical Threat } \\
\text { Words }\end{array}$ & $\begin{array}{c}\text { e - Positive } \\
\text { Words }\end{array}$ \\
\hline $\begin{array}{c}\text { Mean } \\
\text { Imageability }\end{array}$ & $\begin{array}{c}3.67 \\
1.50 \\
\end{array}$ & $\begin{array}{c}3.18 \\
.74\end{array}$ & $\begin{array}{c}\mathbf{3 . 1 8 ^ { \mathrm { e } }} \\
.03\end{array}$ & $\begin{array}{c}\mathbf{5 . 4 4} \\
.45 \\
\end{array}$ & $\begin{array}{c}4.17^{\mathrm{c}} \\
.65\end{array}$ \\
\hline $\begin{array}{c}\text { Mean } \\
\text { Valence }\end{array}$ & $\begin{array}{c}\mathbf{3 . 3 0}^{\text {bcde }} \\
.15\end{array}$ & $\begin{array}{c}\mathbf{2 . 6 5 ^ { \text { acde } }} \\
.39\end{array}$ & $\begin{array}{c}\mathbf{1 . 8 0}^{\text {abe }} \\
.11\end{array}$ & $\begin{array}{c}\mathbf{1 . 4 0} \\
.17\end{array}$ & $\begin{array}{c}\mathbf{4 . 6 5} \\
.12\end{array}$ \\
\hline
\end{tabular}

Note. For each word type, the mean rating for each dimension is shown in bold font, with the standard deviation in plain font beneath. Superscripts beside each mean indicate the other word types that significantly differ from that word type at the $p<.05$ level: ${ }^{\mathrm{a}}$ neutral words, ${ }^{\mathrm{b}}$ uncertain words, ${ }^{\mathrm{c}}$ social threat words, ${ }^{\mathrm{d}}$ physical threat words, ${ }^{\mathrm{e}}$ positive words. A lack of superscript indicates no significant difference between that word type and other types. Due to heterogeneity of variance, Games-Howell pairwise comparisons were used. 
In Set 1, ratings of uncertainty were significantly different across word categories, Welch's $F(4,13.00)=39.31, p<.05$. Pairwise comparisons showed that uncertain words were significantly higher on uncertainty than all the other categories of words. In addition, social threat and physical threat words were higher on uncertainty than positive words. A similar pattern of results for uncertain words was seen in Set $2 F(4,24)=$ $148.11, p<.05$, as uncertain words were significantly higher on uncertainty than all other types of words (note: Welch's $F$ could not be computed due to zero variance in social threat word ratings). As with Set 1, social threat words were higher on uncertainty than positive words; however, physical threat words were only higher on uncertainty than neutral words (see Figures $1 \& 2$ ).

In Set 1, analyses with valence as the outcome showed significant differences between word types, Welch's $F(4,13.28)=300.53, p<.05$. Specifically, positive words were significantly more positive compared to all other word types. Furthermore, social and physical threat words were significantly more negative compared to other word types. There were no significant differences in the valence of social threat and physical threat words. Neutral and uncertain words fell in the middle of the valence continuum. The same pattern of results emerged for Set 2, Welch's $F(4,4.28)=268.99, p<.05$, with the addition that neutral words were significantly more positive in valence than uncertain words (see Figures 1 \&2). 


\section{Discussion}

In this study, a large pool of words was generated and pilot tested to obtain ratings on five dimensions of interest. Based on these ratings, a final stimulus set of 64 words was selected, including categories of neutral, uncertain, threat (social and physical), and positive words. Analyses showed that the stimulus words had good construct validity; i.e., they showed a significant relationship with the key characteristic of their respective categories. Specifically, uncertain words were highest as a group on uncertainty; positive words were highest (most positive) on valence; and threat words-both social and physical-lowest (most negative) on valence. Neutral and uncertain words were neutral in valence. In addition, none of the word categories differed significantly with regards to familiarity. While the goal was to achieve similar equivalence in the remaining dimensions, results showed that uncertain words were less imageable, and physical threat words more imageable, than other word types. Similarly, uncertain words were significantly less concrete, and physical threat words more concrete, than all other word types. Given that findings concerning concreteness and imageability were not consistent across word sets, however, these last findings may not be robust. In contrast, ratings of familiarity, uncertainty, and valence were consistent across word sets.

Despite the inconsistency in the concreteness and imageability findings, it is possible that differences between uncertain and physical threat words do exist. While it was hoped that stimuli could be matched on concreteness and imageability, this may not have been realistic given the types of word categories in this study. For example, matching the low imageability and concreteness of abstract, uncertain words (i.e., 'puzzling') to physical threat words (i.e., 'mutilated'), may not be possible. In fact, closer 
matching on these characteristics may compromise the construct validity of uncertain and threat words. For the purposes of this study, therefore, equivalence on concreteness and imageability across categories can be considered as secondary, compared to the primary goal of high construct validity of words within each category.

A second caveat to this study is the sample used, which was small and largely comprised of female Psychology students. Generalizability of the findings to other populations may therefore be limited. In addition, because the words were divided into two sets, only 25 participants rated any given word. It is worth noting, however, that this resulted in 125 ratings of each word, when the five dimensions are taken into account. This can be contrasted with many previous studies, in which stimulus properties received little or no examination.

In conclusion, the current study successfully produced a set of stimulus words with good construct validity and equivalent word familiarity, for use in future research. Differences with regards to the concreteness and imageability of uncertain and physical threat words emerged, which will be taken into account when interpreting future results. 


\section{STUDY 2}

\section{Introduction}

Using the stimulus sets created in Study 1, the goal of Study 2 was to explore the relationship between IU and explicit memory for threatening and uncertain stimuli. As already noted, existing research does not provide strong support for an explicit memory bias for threat among worriers. Nevertheless, a few studies of GAD patients have found an explicit memory bias for threat. Furthermore, studies with high trait anxious participants suggest that this type of bias may in fact characterize anxious individuals. It is also possible that some previous non-significant findings have been due to problems with the validity and specificity of the stimuli employed in those studies. Therefore, the goal of Study 2 was to re-examine the question of explicit memory bias for threat, and more specifically the contribution of IU, while using the stimuli created in Study 1. In this study, a non self-referent explicit encoding task was used to promote incidental learning. A self-referent task was not used because previous research suggests that it does not enhance explicit memory for threat among worriers (i.e., GAD patients: Becker et al., 1999; Bradley et al., 1995; Coles et al., 2007; Mathews, Mogg, et al., 1989; Mogg et al., 1987). Following the incidental task, participants were given two explicit memory tests: free recall and recognition. Free recall is a widely used test of explicit memory. A subsequent recognition memory test was also administered. Because participants are provided with the stimuli on a recognition test, it can be considered a less difficult test of memory than recall, in which participants must independently retrieve information from memory. As a less difficult test, recognition may reveal memory biases that are not evident in free recall. Furthermore, recognition performance can be used to calculate 
sensitivity, which is essentially a measure of a participant's ability to accurately discriminate previously viewed stimuli from distractors; this was calculated in Study 2 (see Results: Calculation of memory outcomes). Given the good construct validity and matching of the stimuli used in this study, it was hypothesized that IU would be a significant predictor of memory bias for threatening and uncertain words. Further exploratory analyses were conducted to assess the impact of worry, cognitive avoidance, and depression on explicit memory.

\section{Method}

\section{Participants}

For this study, participants were recruited primarily through Concordia University's Psychology Department Participant Pool, and received one bonus course credit for participating (one credit $=$ a bonus $1 \%$ of their final grade). Posters were also used to recruit participants from the Montreal community; these individuals were compensated \$10. All methods of recruitment were approved by Concordia University’s Human Research Ethics Committee.

Participants were eligible for the study if they were between 18-65 years of age, could speak and read English fluently, and had not taken part in Study 1.

Ninety-one participants completed the study. After testing, two participants reported that they were not fluent in English and were removed from the sample; another two participants were removed when data screening showed they were significant outliers (see Data Screening and Outlier Analyses). The final sample consisted of 87 individuals, of whom 11 received $\$ 10$ compensation, and 76 received course credit for participation. The sample was made up of 74 women and 13 men, aged 18 to 51, with a mean age of 
$24.45(S D=6.94)$. Ethnicity/race was primarily White $(69 \%)$, followed by Middle Eastern (9\%), Black (6\%), Asian (5\%), Multi-racial (4\%), First Nations (3\%), Other (3\%), and Latino/a (1\%). Ninety percent of the sample were students, with $62 \%$ of the students reporting that they were Psychology majors. Thirty-five percent of the students reported that they were in their first year of university; $42 \%$ were in their second or third year; $5 \%$ were in their fourth year; and 18\% reported their year as Other.

\section{Materials}

The 64 matched stimuli created in Study 1 were used for Study 2. For this study, 32 of the words were presented as stimuli: 8 positive words, 8 neutral words, 8 threat words, and 8 uncertain words. The remaining 32 words ( 8 of each type) were used as distractors during a subsequent recognition test of memory. Note that each set of threat words included an equal number of social and physical threat words.

\section{Procedure}

Presentation of stimulus words. Participants were tested in groups, in a classroom setting. Participants were informed that the study concerned the relationship between how people process words, and their responses to self-report questionnaires. Participants were given consent forms that described the study and conditions of informed consent (see Appendix K ). However, they were not informed in advance that their memory would be tested, as this might have led them to use memorization strategies, making incidental learning impossible. Participants were given a familiarity rating sheet (see Appendix L), on which there were 3-point Likert scales ranging from 1 $=$ not at all familiar to $3=$ very familiar. Participants were told they would view a series of words projected on a screen, and they would be asked to rate each word on how 
familiar it was using the familiarity sheet. In order to prevent further exposure to the stimuli, the words only appeared on the screen, and not on the rating sheet. .Familiarity was defined as how often participants had seen or come into contact with the word; they were asked to rate familiarity even if they were unsure of a word's meaning.

Word stimuli were projected on a screen at the front of the room with a timed PowerPoint ${ }^{\circledR}$ (Microsoft Corporation) slide show. The words appeared in lowercase black Arial font, size 96, centered on an off-white screen. Each word was presented for 8 seconds, after which a tone sounded and a new word appeared. Participants were informed that the tone signaled the appearance of a new word, but they were asked to stay focussed on the screen as the presentations would occur quickly. The familiarity ratings were essentially a filler task whose purpose was to engage participants with the words and promote incidental learning.

Distractor task. Following the presentation of the stimulus words, participants completed a three minute distractor task to minimize recency effects. They were given a lined sheet with a number (841) in the top right corner. They were asked to subtract three from this number, and then subtract three from the result, and then continue subtracting by threes. They were asked to work as quickly and accurately as possible, and to stop when a tone sounded at the end of three minutes.

Free recall task. After the distractor task, participants were given a blank sheet of paper and asked to write down as many words as they could remember, in any order, from the presentation. Participants were given five minutes to recall words.

Recognition task. Following the free recall task, participants were given a sheet containing the 32 words they had viewed during the presentation, mixed in randomized 
order with 32 distractor words. For each word, participants were instructed to make a check mark under "yes" if they thought the word had been presented earlier, and a check under "no" if they did not think it had been presented. They were asked to follow their first guess if they were unsure (see Appendix M for Recognition Sheet).

Completion of self-report questionnaires. Participants were then asked to complete a package of questionnaires, which were in pseudo-randomized order (4 orders were used). However, all packages presented the SUDS ratings on the first page, in order to obtain mood ratings immediately after the tasks described above. There was no time limit for completion of the questionnaires, but most participants took 20 - 30 minutes to complete the package.

Debriefing. Following the completion of the questionnaires, participants were verbally debriefed, and then given a written Debriefing sheet (see Appendix N) concerning the purpose of the study. Because debriefing sheets could easily be left in public areas on campus, and it was critical that future participants remain unaware of the memory test, no specific mention of memory was made in the debriefing sheets. However, this aspect of the research was fully explained during verbal debriefing. Before leaving, participants were compensated for their participation $(\$ 10$ or 1 bonus course credit).

\section{Measures}

The Intolerance of Uncertainty Scale (IUS; French version: Freeston et al., 1994; English translation: Buhr \& Dugas, 2002) is a 27-item self-report measure of a dispositional characteristic associated with negative beliefs about uncertainty and its implications. Items on the IUS are rated on a 5-point Likert scale ranging from $1=$ not at 
all characteristic of me, to $5=$ entirely characteristic of me. The English translation of the IUS has excellent internal consistency, $\alpha=.94$, and good test-retest reliability over five weeks, $r=.074$; it has also shown convergent and divergent validity with regards to measures of worry, anxiety, and depression, and criterion validity with regards to GAD status by questionnaire (Buhr \& Dugas, 2002). A recent large-scale factor analysis suggests that the IUS can be understood in terms of two factors: 1) Uncertainty has negative behavioural and self-referent implications; 2) Uncertainty is unfair and spoils everything (Sexton \& Dugas, 2009a). As expected, both factors have a similarly strong relationship with worry; however, a stronger correlation between Factor 1 and measures of depression, trait anxiety, and GAD analogue status provides support for the distinction between Factor 1 and Factor 2 (Sexton \& Dugas, 2009a). While item loadings on the two factors vary somewhat across cultural groups, the IUS maintains excellent validity and reliability (Norton, 2005; Sexton \& Dugas, 2007; see Appendix O).

The Cognitive Avoidance Questionnaire (CAQ; French version: Gosselin et al., 2002; English translation: Sexton \& Dugas, 2008) is a 25-item self-report measure of five different forms of cognitive avoidance: thought substitution; transformation of images into thoughts; distraction; avoidance of threatening stimuli; and thought suppression. These comprise five CAQ subscales, with five items each, rated on a 5-point Likert scale ranging from $1=$ not at all typical, to $5=$ completely typical. Factor analysis supports the five subscale structure of the CAQ (Sexton \& Dugas, 2008). The CAQ has shown excellent internal consistency for both the total score, $\alpha=.95$, as well as for the subscales, and demonstrates good test-retest reliability over 4 to 6 weeks, $r=.85$ (Sexton 
\& Dugas, 2008). The CAQ shows convergent and divergent validity with regards to measures of worry, thought suppression, and coping styles (Sexton \& Dugas, 2008).

The Dysfunctional Attitudes Scale (DAS; Weissman, 1980) is a questionnaire that measures a set of attitudes related to depression. The DAS has 40 items that describe different attitudes or beliefs. The items represent seven value systems including approval, autonomy, love, achievement, entitlement, perfectionism, and omnipotence. Respondents are asked whether each item is typical of your way of looking at things, rating each item on a 7 -point Likert type scale ranging from $1=$ totally agree to $7=$ totally disagree. The DAS has high convergent validity with regards to measures of depression and depressogenic thinking. The DAS has excellent internal consistency, $\alpha=.82-.94$, as well as high test-retest reliability over eight weeks, $r=.80-.84$ (Weissman, 1980).

The Social Desirability Scale (SDS; Reynolds, 1982) is a measure of the tendency to self-report in a socially favourable manner, and is a short form of the Marlowe-Crowne Social Desirability Scale (Crowne \& Marlowe, 1960). The SDS was included to assess the potential impact of social desirability on responses to study tasks. Thirteen scale items describe socially desirable behaviours and attitudes that have a low incidence of occurrence; participants respond that each item is either true or false for them. The SDS shows good internal consistency (Kuder-Richardson formula 20 reliability $=.76$ ), comparable to that of the full Marlowe Crowne scale (Reynolds, 1982). The SDS also demonstrates convergent validity with other measures of social desirability (Reynolds, 1982).

The Penn State Worry Questionnaire (PSWQ; Meyer, Miller, Metzger, \& Borkovec, 1990) is a widely used self-report measure of excessive worry. Sixteen items 
are rated on a 5-point Likert scale ranging from $1=$ not at all typical to $5=$ very typical . The PSWQ has excellent internal consistency, $\alpha=.86$ to .95 , and good test-retest reliability over 4 weeks, ranging from $r=.74$ to .93 (Meyer et al., 1990; Molina \& Borkovec, 1994). The PSWQ has shown evidence of convergent and divergent validity with regards to worry processes, anxiety, and depression (Brown, Antony, \& Barlow, 1992; Molina \& Borkovec, 1994). The PSWQ also shows sensitivity and specificity when used as a GAD screen (Behar, Alcaine, Zuellig, \& Borkovec, 2003).

The Worry and Anxiety Questionnaire (WAQ; Dugas et al., 2001) is an 11 item self-report questionnaire that assesses DSM-IV-TR (APA, 2000) diagnostic criteria for GAD, including a subscale measuring the severity of GAD somatic symptoms. The WAQ allows respondents to list up to six worry topics. They then rate the degree to which their worry is excessive (on a 9-point Likert scale ranging from $0=$ not at all excessive to $8=$ totally excessive), difficult to control (from $0=$ no difficulty to $8=$ extreme difficulty), and interfering with their lives (from $0=$ not at all to $8=$ very severely). Respondents also rate the frequency of their worry over the past six months (from $0=$ never to $8=$ every day), and the severity of six GAD somatic symptoms on a 9-point Likert scale ranging from $0=$ not at all to $8=$ very severely. The WAQ therefore provides a concise assessment of DSM-IV-TR symptom, severity, and interference criteria for GAD. The WAQ has demonstrated both sensitivity and specificity when used to screen for GAD, and has good test-retest reliability after 9 weeks (Dugas et al., 2001; see Appendix P).

The State-Trait Anxiety Inventory-Trait version, Form Y (STAI-T:

Spielberger, Gorsuch, Lushene, Vagg, \& Jacobs, 1977) is a well-known questionnaire comprised of two scales, one measuring state anxiety, and the other measuring trait 
anxiety. For this study, only trait anxiety was assessed. The STAI-T includes 20 items rated on a 4-point Likert scale ranging from $1=$ almost never to $4=$ almost always. The STAI-T has shown good internal consistency in clinical $(\alpha=.89$; Bieling, Antony, $\&$ Swinson, 1998) and student samples ( $\alpha=.81$; Bernstein $\&$ Eveland, 1982). In a student sample, the STAI-T has shown good test-retest reliability, ranging from $r=.71$ to .75 over 30 days, and $r=.65$ to .68 over 60 days (Spielberger et al., 1977). The STAI-T also demonstrates convergent validity with other measures of anxiety (Bieling et al., 1998; Creamer, Foran, \& Bell, 1995).

The Center for Epidemiologic Studies Depression Scale (CES-D; Radloff, 1977 ) is a self-report measure of depressive symptoms experienced over the past week, designed for use in the general population. Twenty items are rated on a 4-point Likert scale ranging from $0=$ rarely or none of the time (less than 1 day) to $3=$ most or all of the time (5-7 days). The CES-D has excellent internal consistency, and shows convergent and divergent validity with regards to measures of negative affect, interviewer ratings of depression, social functioning, and positive affect (Radloff, 1977). While four factors emerged from a factor analysis (depressed affect; positive affect; somatic and retarded activity; interpersonal), the high internal consistency led the author to recommend the use of the total score for research purposes. When re-administered at 3, 6, and 12 months, the CES-D demonstrated acceptable test-retest reliability, mostly ranging from $r=.45$ to .70 , with higher correlations for shorter time periods (Radloff, 1977).

A Subjective Units of Distress Scale (SUDS) was created in order to assess participants' self-reported mood state immediately after completing the computer tasks. Five mood states are assessed: anxiety, sadness, irritability, well-being, and fatigue. 
Respondents rate their current level of each mood on a scale from 0 to 100 , with 0 representing none, and 100 extreme, levels of the mood state (see Appendix Q).

A General Information Sheet was created to obtain demographic information about participants, including age, sex, education, student status/field of study, language, and race/ethnicity (see Appendix R).

\section{Results}

\section{Data Screening and Outlier Analyses}

The data were double-entered, compared, and corrected to ensure accuracy. A $z$ score was computed for all study variables, and any score greater than \pm 3.29 was considered an outlier (Tabachnik \& Fidell, 2001). Results showed that four participants were univariate outliers on age, but as this is a participant characteristic, none of these were excluded. One participant was an outlier on their total recognition score, $z=-4.01$; as recognition scores are used to calculate two key outcomes for this study (recognition and sensitivity), this participant was removed from the sample. Another participant was an outlier on SUDS sadness ratings $(z=3.61)$, and was also removed. To screen for multivariate outliers, all study variables (except age, which was a univariate outlier) were then entered into a linear regression. Mahalanobis values showed that five participants were multivariate outliers, with scores greater than the Chi-square critical value, $\chi^{2}(16)=$ 26.30, $p<.05$. However, all of these outliers had Cook's distances less than one (for the whole sample, Cook's distances ranged from .00 to .26 ), indicating they would not significantly affect a regression analysis (Tabachnik \& Fidell, 2001); these participants were therefore retained. 
$Z$-scores for skewness were computed by dividing skewness by its standard error; values greater than 2.58 were considered significantly skewed (Field, 2005). According to this criterion, scores on the CAQ were significantly positively skewed $(z=4.91)$, as were scores on the DAS $(z=2.84)$. In addition, three of the Subjective Units of Distress (SUDS) scores were significantly skewed: SUDS sadness $(z=4.12)$ and irritability $(z=$ 5.19) were positively skewed, whereas SUDS well-being $(z=-4.36)$ was negatively skewed. As this pattern on SUDS ratings, DAS and CAQ scores would be expected in a non-clinical sample, and because normality of predictors is not a necessary assumption for regression (Field, 2005, p. 170), none of these variables were transformed. Means and standard deviations for Study 2 measures are presented in Table 2, and Pearson correlations in Table 3.

\section{Calculation of Memory Outcomes}

For this study, two indices of memory were calculated, based on responses to the free recall and recognition memory tasks. Free recall scores were the total number of words of each type (neutral, uncertain, threat, and positive) correctly recalled by each participant. Sub-totals of the number of social and physical threat words recalled were also calculated, but these were primarily used in follow up analyses. Responses on the recognition test were then used to calculate sensitivity (or $d^{\prime}$ ) scores. Sensitivity incorporates two types of information available from a recognition memory test: hits and false alarms. Hits occur when a word is correctly classified, as either a stimulus word (i.e., previously viewed) or a distractor word (new). False alarms occur when a distractor 
Table 2

Means and Standard Deviations for Study 2 Measures

\begin{tabular}{|c|c|c|c|}
\hline Measure & $n$ & $M$ & $S D$ \\
\hline Center for Epidemiologic Studies Depression Scale & 87 & 13.39 & 9.07 \\
\hline Cognitive Avoidance Questionnaire & 87 & 51.64 & 19.12 \\
\hline CAQ Avoidance & 87 & 10.08 & 5.13 \\
\hline CAQ Distraction & 87 & 11.33 & 4.62 \\
\hline CAQ Substitution & 87 & 8.86 & 4.12 \\
\hline CAQ Suppression & 87 & 12.17 & 4.51 \\
\hline CAQ Transformation & 87 & 9.22 & 4.94 \\
\hline Dysfunctional Attitudes Scale & 87 & 120.13 & 32.61 \\
\hline Intolerance of Uncertainty Scale & 86 & 53.69 & 16.88 \\
\hline IUS Factor 1 & 87 & 27.13 & 9.72 \\
\hline IUS Factor 2 & 86 & 26.52 & 8.69 \\
\hline Penn State Worry Questionnaire & 87 & 45.51 & 13.82 \\
\hline Social Desirability Scale & 87 & 6.02 & 2.73 \\
\hline State-Trait Anxiety Inventory-Trait version & 87 & 40.24 & 10.38 \\
\hline SUDS Anxiety & 87 & 31.47 & 24.48 \\
\hline SUDS Fatigue & 87 & 43.13 & 27.68 \\
\hline SUDS Irritability & 87 & 20.10 & 24.10 \\
\hline SUDS Sadness & 87 & 18.31 & 20.56 \\
\hline SUDS Well-being & 86 & 67.98 & 21.83 \\
\hline Worry and Anxiety Questionnaire & 87 & 34.10 & 15.69 \\
\hline
\end{tabular}

Note. $\mathrm{CAQ}=$ Cognitive Avoidance Questionnaire: Avoidance $=$ Avoidance of threatening stimuli subscale; Distraction $=$ Distraction subscale; Substitution $=$ Thought substitution subscale; Suppression $=$ Thought suppression subscale; Transformation $=$ Transformation of images into thoughts subscale. IUS = Intolerance of Uncertainty Scale. SUDS = Subjective Units of Distress Scale. 
Table 3

Correlations Between Study 2 Measures

\begin{tabular}{|c|c|c|c|c|c|c|c|c|c|c|c|c|c|}
\hline & CAQ & CES & DAS & IUS & PSW & SDS & STA & $\mathrm{SUa}$ & SUf & SUi & SUs & SUw & WAQ \\
\hline CAQ & & $.39^{* *}$ & .17 & $.48^{* * a}$ & .12 & -.16 & $.38^{* *}$ & .12 & .08 & $.30^{* *}$ & $.36^{* *}$ & $-.12^{\mathrm{a}}$ & $.41^{* *}$ \\
\hline CES & & & $.58^{* *}$ & $.59^{* * a}$ & $.55^{* *}$ & -.05 & $.80^{* *}$ & $.29^{* *}$ & $.33^{* *}$ & $.30^{* *}$ & $.55^{* *}$ & $-.45^{* * a}$ & a $.63^{* *}$ \\
\hline DAS & & & & $.57^{* * a}$ & $.47^{* *}$ & $-.32^{* *}$ & $.66^{* *}$ & .14 & .12 & .12 & .19 & $-.27^{* a}$ & $.30^{* *}$ \\
\hline IUS & & & & & $.45^{* * a}$ & $-.23^{* a}$ & $.69^{* * a}$ & $.12^{\mathrm{a}}$ & $.22^{* \mathrm{a}}$ & $.20^{\mathrm{a}}$ & $.30^{* * a}$ & $-.10^{b}$ & $.54^{* * \mathrm{a}}$ \\
\hline PSW & & & & & & -.16 & $.64^{* *}$ & $.39^{* *}$ & $.39^{* *}$ & $.27^{*}$ & .29 & $-.42^{* * a}$ & a $.66^{* *}$ \\
\hline SDS & & & & & & & $-.23^{*}$ & .13 & -.17 & -.16 & -.02 & $-.12^{\mathrm{a}}$ & -.17 \\
\hline STA & & & & & & & & $.25^{*}$ & $.39^{* *}$ & $.28^{* *}$ & $.45^{* *}$ & $-.42^{* * a}$ & a $.64^{* *}$ \\
\hline SUa & & & & & & & & & $.47^{* *}$ & $.55^{* *}$ & $.52^{* *}$ & $-.02^{\mathrm{a}}$ & $.26^{*}$ \\
\hline SUf & & & & & & & & & & $.50^{* *}$ & $.48^{* *}$ & $-.08^{\mathrm{a}}$ & $.42^{* *}$ \\
\hline SUi & & & & & & & & & & & $.59^{* *}$ & $-.14^{\mathrm{a}}$ & $.25^{*}$ \\
\hline SUs & & & & & & & & & & & & $-.22^{* a}$ & $.38^{* *}$ \\
\hline SUw & & & & & & & & & & & & & $-.28^{* *}$ \\
\hline WAQ & & & & & & & & & & & & & \\
\hline
\end{tabular}

Note. $n=87$, except : ${ }^{\mathrm{a}} n=86 ;{ }^{\mathrm{b}} n=85$. CAQ $=$ Cognitive Avoidance Questionnaire; CES = Center for Epidemiologic Studies Depression scale; DAS = Dysfunctional Attitudes Scale; IUS = Intolerance of Uncertainty Scale; PSW = Penn State Worry Questionnaire; SDS = Social Desirability Scale; STA = State-Trait Anxiety Inventory-Trait version; $\mathrm{SU}=$ Subjective Units of Distress: $\mathrm{a}=\mathrm{anxiety}, \mathrm{f}=$ fatigue, $\mathrm{i}=$ irritability, $\mathrm{s}=$ sadness, $\mathrm{w}=$ well-being; $\mathrm{WAQ}=$ Worry and Anxiety Questionnaire. ${ }^{*} p<.05 .{ }^{* *} p<.01$. 
word is incorrectly classified as a stimulus word. Sensitivity accounts for both hits and false alarms, thereby providing a more exact index of recognition memory. For example, a participant may have a high recognition score, but if their false alarm rate is correspondingly high, their score may represent a tendency to over-endorse test items. Sensitivity essentially provides a measure of participants' ability to accurately distinguish stimuli from distractors on a recognition test. Statistically, sensitivity can be defined as the standardized difference between the distribution of hits and the distribution of false alarms, such that: $\mathrm{d}^{\prime}=z$ (false alarms) $-z$ (hits; Stanislaw and Todorov, 1999). In order to avoid computational problems due to extreme hit and false alarm scores, a transformation was first performed. The loglinear transformation recommended by Hautus (1995) was applied, in which 0.5 is added to all false alarm and hit scores, and 1 is added to the number of possible stimuli as well as the number of distractor items. A d' score was then calculated for each word type, including sub-categories of social and physical threat.

\section{Manipulation Checks}

Frequencies of hits on the recognition memory test showed that there were no stimulus words nor distractor words with floor or ceiling effects (i.e., there were no words with either $0 \%$ or $100 \%$ hits). On the recognition test, a paired samples $t$-test showed that the mean hit rate $(M=27.10, S E=.34)$ was significantly higher than the false alarm rate $(M=3.69, S E=.37, t(86)=47.75, p<.000)$. This finding shows that participants were able to differentiate stimulus from distractor words at a rate significantly better than chance, and suggests that incidental learning of the stimuli did in fact occur. 


\section{Regressions With All Study Variables}

For all regressions, diagnostic indices were first calculated to ensure that the necessary regression assumptions were met (i.e., the impact of outliers and influential cases; generalizability of the model; see Field, 2005, pp. 162-175). Examination of these diagnostics showed that assumptions were adequately met for the regression analyses that follow. Before hypothesis-specific regressions were carried out, regressions with all study variables were conducted to determine which variables might be further investigated. To this end, four regressions were used to predict recall for each type of word (neutral, uncertain, threat, and positive), separately. Predictors were study variables except the Intolerance of Uncertainty Scale, because this would be investigated in the main analyses; predictors were entered in one step.

Results of these analyses showed that for recall, both cognitive avoidance $(\beta=$ $0.47, p=0.001)$ and worry $(\beta=0.38, p=0.039)$ were significant predictors of recall for threat words. However, none of the other study variables were significant predictors of recall for neutral, uncertain, or positive words.

The same procedure was then followed, but with recognition sensitivity for each word type as the outcome. Results indicated that SUDS anxiety ratings were a significant predictor of sensitivity for uncertain words ( $\beta=0.52, p=0.002)$, whereas SUDS irritability $(\beta=-0.45, p=0.005)$ and social desirability $(\beta=-0.27, p=0.046)$ were significant negative predictors. For positive words, results showed that female sex was a significant predictor $(\beta=-0.28, p=0.017)$, as were SUDS anxiety ratings $(\beta=0.53, p=$ $0.001)$; SUDS irritability $(\beta=-0.31, p=0.049)$ and SUDS fatigue ratings $(\beta=-0.30, p=$ 
0.048 ) were significant negative predictors of sensitivity for positive words. No other variables were significant predictors of sensitivity for neutral or threat words.

Given these results, sex, social desirability, SUDS anxiety, and SUDS irritability were entered in the first steps of subsequent regressions. Findings concerning cognitive avoidance and worry were examined in separate follow up analyses. Findings concerning SUDS fatigue were not further explored in order to limit the number of analyses.

\section{Intolerance of Uncertainty and Recall}

In order to address study hypotheses concerning intolerance of uncertainty and recall for different types of words, a series of hierarchical regressions were conducted. Recall for neutral, uncertain, threat, and positive words were examined in four separate analyses. As sex, social desirability, SUDS anxiety, and SUDS irritability were identified as variables of interest, these were entered as predictors in Step 1. Scores on the Intolerance of Uncertainty Scale (IUS) were then entered in Step 2, to determine whether intolerance of uncertainty would make a significant contribution to recall, above and beyond sex, social desirability, SUDS anxiety, and SUDS irritability.

Results showed that none of these variables were significant predictors of recall for neutral, uncertain, threat, or positive words (see Appendices S, T, U, V).

\section{Intolerance of Uncertainty and Recognition Sensitivity (d')}

Another four regressions were then carried out to assess the relationship between intolerance of uncertainty and sensitivity for the four types of words. As before, a separate regression was carried out for sensitivity for each word type; sex, social desirability, SUDS anxiety, and SUDS irritability were entered in Step 1, and the IUS in Step 2. 
For uncertain words, results indicated that at all steps of the model, SUDS anxiety was a significant predictor of sensitivity, whereas SUDS irritability was a significant negative predictor. For threat words, SUDS anxiety was a significant predictor at all steps of the model. SUDS anxiety was also a significant predictor of sensitivity for positive words at all steps, and SUDS irritability was a significant negative predictor at all steps of the model. Female sex was a significant predictor of sensitivity for positive words at Step 1, and social desirability was a significant negative predictor at Step 1 . None of the variables were predictors of sensitivity for neutral words (see Tables 4 - 7). 
Table 4

Study 2: Hierarchical Regression with Sex, Social Desirability, Mood, and Intolerance of Uncertainty Predicting Sensitivity for Neutral Words

\begin{tabular}{|c|c|c|c|c|c|}
\hline Predictor & $\Delta R^{2}$ & $B$ & $S E B$ & $\beta$ & $95 \% \mathrm{CI}$ \\
\hline Step 1 & .03 & & & & \\
\hline Constant & & 2.40 & .20 & & {$[2.00,2.79]$} \\
\hline Sex & & .02 & .20 & .01 & {$[-.37, .41]$} \\
\hline SDS & & -.04 & .03 & -.15 & {$[-.09, .02]$} \\
\hline SUDSanx & & .00 & .00 & .11 & {$[-.00, .01]$} \\
\hline SUDSirr & & .00 & .00 & -.03 & {$[-.01, .01]$} \\
\hline
\end{tabular}

Step 2

.01

$\begin{array}{lrrrr}\text { Constant } & 2.19 & .33 & & {[1.54,2.83]} \\ \text { Sex } & .03 & .20 & .02 & {[-.37, .43]} \\ \text { SDS } & -.03 & .03 & -.13 & {[-.09, .03]} \\ \text { SUDSanx } & .00 & .00 & .10 & {[-.00, .01]} \\ \text { SUDSirr } & -.00 & .00 & -.04 & {[-.01, .01]} \\ \text { IUS } & .00 & .00 & .10 & {[-.01, .01]}\end{array}$

Note. $n=86 . \mathrm{CI}=$ confidence interval for $B . \mathrm{SDS}=$ Social Desirability Scale; $\mathrm{SUDS}=$ Subjective Units of Distress: anx = anxiety, irr = irritability; IUS = Intolerance of Uncertainty Scale. 
Table 5

Study 2: Hierarchical Regression with Sex, Social Desirability, Mood, and Intolerance of Uncertainty Predicting Sensitivity for Uncertain Words

\begin{tabular}{|c|c|c|c|c|c|}
\hline Predictor & $\Delta R^{2}$ & $B$ & $S E B$ & $\beta$ & $95 \% \mathrm{CI}$ \\
\hline Step 1 & $.11^{*}$ & & & & \\
\hline Constant & & 2.28 & .20 & & {$[1.89,2.68]$} \\
\hline Sex & & -.21 & .20 & -.11 & {$[-.61, .19]$} \\
\hline SDS & & -.04 & .03 & -.17 & {$[-.10, .01]$} \\
\hline SUDSanx & & .01 & .00 & $.37 * *$ & {$[.00, .02]$} \\
\hline SUDSirr & & -.01 & .00 & $-.36 * *$ & {$[-.02,-.00]$} \\
\hline
\end{tabular}

Step 2

.01

$\begin{array}{lcccc}\text { Constant } & 2.10 & .33 & & {[1.44,2.75]} \\ \text { Sex } & -.20 & .20 & -.11 & {[-.60, .20]} \\ \text { SDS } & -.04 & .03 & -.15 & {[-.09, .02]} \\ \text { SUDSanx } & .01 & .00 & .36^{* *} & {[.00, .02]} \\ \text { SUDSirr } & -.01 & .00 & -.37^{* *} & {[-.02,-.00]} \\ \text { IUS } & .00 & .00 & .08 & {[-.01, .01]}\end{array}$

Note. $n=86 . \mathrm{CI}=$ confidence interval for $B . \mathrm{SDS}=$ Social Desirability Scale; SUDS $=$ Subjective Units of Distress: anx = anxiety, irr = irritability; IUS = Intolerance of Uncertainty Scale.

$* p<.05 ; * * p<.01$. 
Table 6

Study 2: Hierarchical Regression with Sex, Social Desirability, Mood, and Intolerance of Uncertainty Predicting Sensitivity for Threat Words

\begin{tabular}{|c|c|c|c|c|c|}
\hline Predictor & $\Delta R^{2}$ & $B$ & $S E B$ & $\beta$ & $95 \% \mathrm{CI}$ \\
\hline Step 1 & .08 & & & & \\
\hline Constant & & 2.37 & .21 & & {$[1.96,2.77]$} \\
\hline Sex & & -.11 & .20 & -.06 & {$[-.52, .30]$} \\
\hline SDS & & -.05 & .03 & -.21 & {$[-.11, .00]$} \\
\hline SUDSanx & & .01 & .00 & $.31 *$ & {$[.00, .02]$} \\
\hline SUDSirr & & -.01 & .00 & -.17 & {$[-.01, .00]$} \\
\hline
\end{tabular}

Step 2

.00

$\begin{array}{lcccc}\text { Constant } & 2.32 & .34 & & {[1.65,2.99]} \\ \text { Sex } & -.11 & .21 & -.06 & {[-.52, .30]} \\ \text { SDS } & -.05 & .03 & -.21 & {[-.11, .01]} \\ \text { SUDSanx } & .01 & .00 & .31^{*} & {[.00, .02]} \\ \text { SUDSirr } & -.01 & .00 & -.17 & {[-.01, .00]} \\ \text { IUS } & .00 & .00 & .02 & {[-.01, .01]}\end{array}$

Note. $n=86 . \mathrm{CI}=$ confidence interval for $B . \mathrm{SDS}=$ Social Desirability Scale; $\mathrm{SUDS}=$ Subjective Units of Distress: anx = anxiety, irr = irritability; IUS = Intolerance of Uncertainty Scale.

$* p<.05$. 
Table 7

Study 2: Hierarchical Regression with Sex, Social Desirability, Mood, and Intolerance of Uncertainty Predicting Sensitivity for Positive Words

\begin{tabular}{|c|c|c|c|c|c|}
\hline Predictor & $\Delta R^{2}$ & $B$ & $S E B$ & $\beta$ & $95 \% \mathrm{CI}$ \\
\hline Step 1 & $.15^{*}$ & & & & \\
\hline Constant & & 2.21 & .25 & & {$[1.71,2.70]$} \\
\hline Sex & & -.51 & .25 & $-.22 *$ & {$[-1.00,-.02]$} \\
\hline SDS & & -.08 & .03 & -.24 & {$[-.14,-.01]$} \\
\hline SUDSanx & & .01 & .00 & .39 & {$[.01, .02]$} \\
\hline SUDSirr & & -.01 & .01 & $-.35 *$ & {$[-.02,-.00]$} \\
\hline
\end{tabular}

Step 2

.03

$\begin{array}{lllll}\text { Constant } & 1.64 & .40 & & {[.85,2.43]} \\ \text { Sex } & -.48 & .24 & -.20 & {[-.96, .01]} \\ \text { SDS } & -.06 & .03 & -.20 & {[-.13, .01]} \\ \text { SUDSanx } & .01 & .00 & .37^{* *} & {[.00, .02]} \\ \text { SUDSirr } & -.01 & .00 & -.36^{* *} & {[-.02,-.00]} \\ \text { IUS } & .01 & .01 & .19 & {[.00, .02]}\end{array}$

Note. $n=86 . \mathrm{CI}=$ confidence interval for $B . \mathrm{SDS}=$ Social Desirability Scale; $\mathrm{SUDS}=$ Subjective Units of Distress: anx = anxiety, irr = irritability; IUS = Intolerance of Uncertainty Scale.

$* p<.05 ; * * p<.01$. 


\section{Follow-up Analyses: Cognitive Avoidance and Worry}

In earlier analyses with all study variables, cognitive avoidance and worry were significant predictors of recall for threat words; follow-up analyses were therefore carried out to explore these findings. A hierarchical regression was conducted with sex, social desirability, SUDS anxiety, and SUDS irritability as predictors in Step 1; worry as measured by the PSWQ in Step 2, and cognitive avoidance as measured by the CAQ total score in Step 3.

Results showed that in Step 1, SUDS anxiety was a significant predictor of threat word recall $(\beta=0.28, p=0.043)$; however, in the final model, cognitive avoidance was the only significant predictor of threat word recall $(\beta=0.32, p=0.004)$.

As explained in the Measures section, the CAQ can be interpreted both in terms of its total score, and in terms of its five subscales: thought substitution; transformation of images into thoughts; distraction; avoidance of threatening stimuli; and thought suppression. Further analyses were therefore conducted to determine whether recall for threat was related to the specific forms of cognitive avoidance measured by the CAQ subscales. Accordingly, five regressions were carried out, with sex, social desirability, SUDS anxiety, and SUDS irritability in Step 1, and one of the five CAQ subscales in Step 2. In order to prevent problems with collinearity, each of the CAQ subscales was examined separately.

CAQ thought substitution was not a significant predictor of threat word recall; however, SUDS anxiety was a significant predictor at both steps of the model. CAQ transformation of images into thoughts was a significant predictor of threat word recall in Step 2, and SUDS anxiety was a significant predictor at both steps. CAQ distraction was 
also a significant predictor at Step 2 of the model, and SUDS anxiety at Step 1. CAQ avoidance of threatening stimuli was a significant predictor of threat word recall, as was SUDS anxiety in both steps of the model. CAQ thought suppression was also a significant predictor of threat word recall in Step 2; SUDS anxiety was again a significant predictor in both steps (see Tables 8 - 12).

As outlined in Study 1, the set of threat words is comprised of both social and physical threat words. follow-up analyses were therefore performed to explore potential differences between social and physical threat words, with the four CAQ subscales that were significant predictors of threat word recall. To examine recall of social threat words, a regression was carried out with sex, social desirability, SUDS anxiety, and SUDS irritability in Step 1, and one of the CAQ subscales in Step 2; again, each subscale was analyzed separately. The same analyses were then performed, but with recall of physical threat words as the outcome.

For recall of social threat words, in the analysis with CAQ transformation of images into thoughts, SUDS anxiety was the only significant predictor in the final step ( $\beta$ $=0.27, p=0.048$ ). For CAQ avoidance of threatening stimuli, SUDS anxiety was the only significant predictor of recall in Step $2(\beta=0.28, p=0.043)$. Neither CAQ distraction nor CAQ thought suppression were significant predictors of recall for social threat words.

For recall of physical threat words, results showed that CAQ distraction was a significant predictor (Step 2: $\beta=0.32, p=0.004$ ). CAQ avoidance of threatening stimuli was also a significant predictor of physical threat word recall (Step 2: $\beta=0.28, p=$ 0.011). CAQ thought suppression was also a significant predictor of physical threat word 
recall (Step 2: $\beta=0.31, p=0.006)$. CAQ transformation of images into thoughts was not a significant predictor of physical threat word recall. 
Table 8

Study 2: Hierarchical Regression with Sex, Social Desirability, Mood, Worry, and CAQ Thought Substitution Predicting Recall for Threat Words

\begin{tabular}{|c|c|c|c|c|c|}
\hline Predictor & $\Delta R^{2}$ & $B$ & $S E B$ & $\beta$ & $95 \% \mathrm{CI}$ \\
\hline Step 1 & .05 & & & & \\
\hline Constant & & 1.50 & .36 & & {$[.78,2.22]$} \\
\hline Sex & & -.20 & .36 & -.06 & {$[-.92, .52]$} \\
\hline SDS & & -.01 & .05 & -.02 & {$[-.11, .09]$} \\
\hline SUDSanx & & .01 & .01 & $.28 *$ & {$[.00, .03]$} \\
\hline SUDSirr & & -.01 & .01 & -.14 & {$[-.02, .01]$} \\
\hline Step 2 & .00 & & & & \\
\hline Constant & & 1.45 & .44 & & {$[.57,2.33]$} \\
\hline Sex & & -.19 & .37 & -.06 & {$[-.92, .53]$} \\
\hline SDS & & -.01 & .05 & -.02 & {$[-.11, .09]$} \\
\hline SUDSanx & & .01 & .01 & $.28 *$ & {$[.00, .03]$} \\
\hline SUDSirr & & -.01 & .01 & -.15 & {$[-.02, .01]$} \\
\hline CAQsub & & .01 & .03 & .02 & {$[-.06, .07]$} \\
\hline
\end{tabular}

Note. $n=87 . \mathrm{CI}=$ confidence interval for $B . \mathrm{SDS}=$ Social Desirability Scale; SUDS $=$ Subjective Units of Distress: anx = anxiety, irr = irritability; PSWQ = Penn State Worry Questionnaire; CAQsub = Cognitive Avoidance Questionnaire, thought substitution subscale.

$* p<.05$. 
Table 9

Study 2: Hierarchical Regression with Sex, Social Desirability, Mood, Worry, and CAQ Transformation of Images into Thoughts Predicting Recall for Threat Words

\begin{tabular}{|c|c|c|c|c|c|}
\hline Predictor & $\Delta R^{2}$ & $B$ & $S E B$ & $\beta$ & $95 \% \mathrm{CI}$ \\
\hline Step 1 & .05 & & & & \\
\hline Constant & & 1.50 & .36 & & {$[.78,2.22]$} \\
\hline Sex & & -.20 & .36 & -.06 & {$[-.92, .52]$} \\
\hline SDS & & -.01 & .05 & -.02 & {$[-.11, .09]$} \\
\hline SUDSanx & & .01 & .01 & $.28 *$ & {$[.00, .03]$} \\
\hline SUDSirr & & -.01 & .01 & -.14 & {$[-.02, .01]$} \\
\hline Step 2 & $.06^{*}$ & & & & \\
\hline Constant & & .89 & .43 & & {$[.03,1.76$} \\
\hline Sex & & -.12 & .35 & -.04 & {$[-.82, .58]$} \\
\hline SDS & & .00 & .05 & .00 & {$[-.09, .10]$} \\
\hline SUDSanx & & .01 & .01 & $.30 *$ & {$[.00, .03]$} \\
\hline SUDSirr & & -.01 & .01 & -.23 & {$[-.02, .00]$} \\
\hline CAQtrans & & .06 & .03 & $.27 *$ & {$[.01, .12]$} \\
\hline
\end{tabular}

Note. $n=87 . \mathrm{CI}=$ confidence interval for $B . \mathrm{SDS}=$ Social Desirability Scale; SUDS $=$ Subjective Units of Distress: anx = anxiety, irr = irritability; PSWQ = Penn State Worry Questionnaire; CAQtrans = Cognitive Avoidance Questionnaire, transformation of images into thoughts subscale.

$* p<.05$. 
Table 10

Study 2: Hierarchical Regression with Sex, Social Desirability, Mood, Worry, and CAQ Distraction Predicting Recall for Threat Words

\begin{tabular}{|c|c|c|c|c|c|}
\hline Predictor & $\Delta R^{2}$ & $B$ & $S E B$ & $\beta$ & $95 \% \mathrm{CI}$ \\
\hline Step 1 & .05 & & & & \\
\hline Constant & & 1.50 & .36 & & {$[.78,2.22]$} \\
\hline Sex & & -.20 & .36 & -.06 & {$[-.92, .52]$} \\
\hline SDS & & -.01 & .05 & -.02 & {$[-.11, .09]$} \\
\hline SUDSanx & & .01 & .01 & $.28 *$ & {$[.00, .03]$} \\
\hline SUDSirr & & -.01 & .01 & -.14 & {$[-.02, .01]$} \\
\hline Step 2 & $.10 * *$ & & & & \\
\hline Constant & & .60 & .45 & & {$[-.29,1.49]$} \\
\hline Sex & & -.12 & .34 & -.04 & {$[-.80, .57]$} \\
\hline SDS & & -.00 & .05 & -.01 & {$[-.10, .09]$} \\
\hline SUDSanx & & .01 & .01 & .23 & {$[-.00, .02]$} \\
\hline SUDSirr & & -.01 & .01 & -.18 & {$[-.02, .00]$} \\
\hline CAQdis & & .08 & .03 & $.33 * *$ & {$[.03, .14]$} \\
\hline
\end{tabular}

Note. $n=87 . \mathrm{CI}=$ confidence interval for $B . \mathrm{SDS}=$ Social Desirability Scale; SUDS $=$ Subjective Units of Distress: anx = anxiety, irr = irritability; PSWQ = Penn State Worry Questionnaire; CAQdis = Cognitive Avoidance Questionnaire, distraction subscale. $* p<.05 ; * * p<.01$. 
Table 11

Study 2: Hierarchical Regression with Sex, Social Desirability, Mood, Worry, and CAQ Avoidance of Threatening Stimuli Predicting Recall for Threat Words

\begin{tabular}{|c|c|c|c|c|c|}
\hline Predictor & $\Delta R^{2}$ & $B$ & $S E B$ & $\beta$ & $95 \% \mathrm{CI}$ \\
\hline Step 1 & .05 & & & & \\
\hline Constant & & 1.50 & .36 & & {$[.78,2.22]$} \\
\hline Sex & & -.20 & .36 & -.06 & {$[-.92, .52]$} \\
\hline SDS & & -.01 & .05 & -.02 & {$[-.11, .09]$} \\
\hline SUDSanx & & .01 & .01 & $.28^{*}$ & {$[.00, .03]$} \\
\hline SUDSirr & & -.01 & .01 & -.14 & {$[-.02, .01]$} \\
\hline
\end{tabular}

Step 2

$.09 * *$

$\begin{array}{lllll}\text { Constant } & .68 & .45 & & {[-.21,1.57]} \\ \text { Sex } & -.17 & .35 & -.05 & {[-.86, .52]} \\ \text { SDS } & .01 & .05 & .01 & {[-.09, .10]} \\ \text { SUDSanx } & .02 & .01 & .32 * & {[.00, .03]} \\ \text { SUDSirr } & -.01 & .01 & -.20 & {[-.02, .00]} \\ \text { CAQav } & .07 & .02 & .31 * * & {[.02, .12]}\end{array}$

Note. $n=87 . \mathrm{CI}=$ confidence interval for $B . \mathrm{SDS}=$ Social Desirability Scale; SUDS $=$ Subjective Units of Distress: anx = anxiety, irr = irritability; PSWQ = Penn State Worry Questionnaire; CAQav = Cognitive Avoidance Questionnaire, avoidance of threatening stimuli subscale. $* p<.05 ; * * p<.01$. 
Table 12

Study 2: Hierarchical Regression with Sex, Social Desirability, Mood, Worry, and CAQ Thought Suppression Predicting Recall for Threat Words

\begin{tabular}{|c|c|c|c|c|c|}
\hline Predictor & $\Delta R^{2}$ & $B$ & $S E B$ & $\beta$ & $95 \% \mathrm{CI}$ \\
\hline Step 1 & .05 & & & & \\
\hline Constant & & 1.50 & .36 & & {$[.78,2.22]$} \\
\hline Sex & & -.20 & .36 & -.06 & {$[-.92, .52]$} \\
\hline SDS & & -.01 & .05 & -.02 & {$[-.11, .09]$} \\
\hline SUDSanx & & .01 & .01 & $.28 *$ & {$[.00, .03]$} \\
\hline SUDSirr & & -.01 & .01 & -.14 & {$[-.02, .01]$} \\
\hline Step 2 & $.10 * *$ & & & & \\
\hline Constant & & .30 & .52 & & {$[-.75,1.34]$} \\
\hline Sex & & -.13 & .35 & -.04 & {$[-.81, .56]$} \\
\hline SDS & & .02 & .05 & .05 & {$[-.07, .12]$} \\
\hline SUDSanx & & .01 & .01 & $.29 *$ & {$[.00, .03]$} \\
\hline SUDSirr & & -.01 & .01 & -.21 & {$[-.02, .00]$} \\
\hline CAQsupp & & .09 & .03 & $.33 * *$ & {$[.03, .14]$} \\
\hline
\end{tabular}

Note. $n=87 . \mathrm{CI}=$ confidence interval for $B . \mathrm{SDS}=$ Social Desirability Scale; SUDS $=$ Subjective Units of Distress: anx = anxiety, irr = irritability; PSWQ = Penn State Worry Questionnaire; CAQsupp = Cognitive Avoidance Questionnaire, thought suppression subscale.

$* p<.05 ; * *<<.01$. 


\section{Discussion}

In this study, the relationship between IU and explicit memory bias for threat and uncertainty was explored. The stimuli piloted in Study 1 were used in an incidental learning task, and explicit memory (recall and recognition sensitivity) for the different types of words was measured. Regressions were then used to assess the contribution of IU to memory for the stimuli; the contribution of factors such as worry, cognitive avoidance, and depression were also assessed.

Exploratory regressions with all study variables showed that worry and cognitive avoidance were predictors of recall, but only for threat words. SUDS anxiety was a significant predictor of sensitivity for uncertain and positive words, and SUDS irritability was a negative predictor for these word types. Social desirability emerged as a negative predictor of sensitivity for uncertain words. Lastly, female sex was a predictor, and SUDS fatigue a negative predictor, of positive word sensitivity.

A series of regressions were then used to test the hypothesis that IU would predict recall and sensitivity for uncertain and threat words. Contrary to expectation, IU was not a predictor of sensitivity, or recall, for any type of word. However, SUDS anxiety was a predictor, and SUDS irritability a negative predictor, of sensitivity for uncertain words. SUDS anxiety was also a predictor of sensitivity for threat words. For positive words, SUDS anxiety and female sex were predictors, whereas social desirability and SUDS irritability were negative predictors.

Based on the analyses with all study variables, follow-up regressions were conducted with worry and cognitive avoidance. In these regressions, SUDS anxiety and cognitive avoidance were significant predictors of threat word recall; however, worry was 
no longer significant. Analyses of specific CAQ subscales showed that transformation of images into thoughts, distraction, avoidance of threatening stimuli, and thought suppression were all predictors of threat word recall; in all cases, SUDS anxiety was also a predictor. Separate analyses of social and physical threat words showed that distraction, avoidance of threatening stimuli, and thought suppression were significant predictors of recall, but for only for physical threat words. For social threat words, SUDS anxiety was the only predictor of recall, in the analyses examining transformation of images into thoughts, and avoidance of threatening stimuli.

The main outcome of Study 2 was the failure to find a relationship between IU and explicit memory for threatening or uncertain words. This contrasts with the study hypotheses, and with three previous studies that have found a relationship between IU and information processing biases. It is important to note, however, that only one study to date has found a relationship between IU and memory bias for uncertain words. In that study, high IU participants recalled a significantly higher proportion of uncertain as compared to neutral words, when contrasted with participants low in IU. However, only two types of words - uncertain and neutral — were presented in that study. In the current study, uncertain words were presented with three other types of words, two of which were emotionally valenced (threat and positive words). It may be that the presence of other valenced words interfered with focussed processing of words denoting uncertainty. In this sense, the use of four word types in this study may have been an overly stringent test of information processing bias. Another possibility is that uncertain words were not preferentially processed due to their lower concreteness and imageability, particularly when contrasted with physical threat words. Imageability and concreteness are both 
factors that can enhance memory; as noted in Study 1, uncertain words were lower on these dimensions than the other types of words.

Another key outcome was that worry was not a significant predictor of memory bias for uncertain words. While there was a significant relationship between worry and recall of threat words in the initial analyses, this relationship became non-significant when cognitive avoidance was included. This suggests that while worry (a symptom) makes a contribution to memory bias, cognitive avoidance (a cognitive process or disposition) may be a better predictor. This was true specifically for physical threat words, and for three particular forms of cognitive avoidance: distraction, avoidance of threatening stimuli, and thought suppression. While there were no specific hypotheses regarding cognitive avoidance, current theory suggests that it should be negatively related to memory bias. Specifically, the tendency to avoid upsetting mental material would be expected to interfere with the processing of that material, and lead to poorer memory. In fact, the opposite relationship was found: cognitive avoidance was positively related to memory for threat. One possible explanation for this is that while the Cognitive Avoidance Questionnaire measures the self-reported tendency to engage in cognitive avoidance strategies, it does not measure how successful these attempts are. For example, an individual may attempt to avoid upsetting thoughts, but paradoxically draw more processing resources to the very content they are trying to avoid. This would be consistent with findings from the thought suppression literature, which suggest that attempts to avoid thoughts can actually result in an increase in those thoughts (see Abramowitz, Tolin, \& Street, 2001, for a review). In the current study, some support for this idea can be drawn from the fact that thought suppression was one of the forms of 
cognitive avoidance related to enhanced memory for threat. However, as cognitive avoidance analyses were strictly exploratory, these outcomes should be interpreted with caution.

Of further interest is that when social and physical threat words were examined separately, cognitive avoidance was related to recall for physical threat words alone. Study 1 analyses showed no significant differences between social and physical threat words on valence, indicating that they are equally negative or "threatening". However, results also suggested that physical threat words were significantly more concrete and imageable than social threat words. Therefore, a memory bias may have emerged for physical threat words because they are higher in imageability and concreteness. Alternatively, these findings may reflect an actual bias toward the construct of physical threat: stimuli denoting physical threat may be preferentially processed and recalled. However, the possibility that results may be due to the higher concreteness and imageability of physical threat words cannot be entirely ruled out.

In the initial exploratory regressions, some other study variables contributed to memory bias. For example, social desirability was negatively related to sensitivity for uncertain and positive words. This implies that individuals high on social desirability are less likely to accurately discriminate between certain types of old and new words on a recognition test. For instance, higher social desirability may lead an unsure participant to endorse more words as previously viewed, because they know their memory is being tested. Why this would only apply to uncertain and positive words, however, is unclear and would require further exploration. Another finding was the positive relationship between state anxiety and sensitivity for all of the non-neutral words. While previous 
research suggests that high state anxiety can interfere with performance, this may primarily be true for individuals who are high in trait anxiety (i.e., Eysenck, Payne, \& Derakshan, 2005). The levels of trait anxiety in this study are relatively low compared to many studies in this area (State-Trait Anxiety Inventory-Trait version: $M=40.24, S D=$ 10.38; range 29.86 - 50.62). Furthermore, the levels of state anxiety in this study are fairly low (SUDS anxiety rating out of 100: $M=31.47, S D=24.48$; range $6.99-55.95$ ). In a relatively low trait anxious sample, such small elevations in state anxiety may reflect slightly heightened arousal, and consequently improved performance on a recognition task. The finding of a negative relationship between irritability and memory performance may initially seem self-explanatory. However, it is more difficult to explain why irritability only negatively affected sensitivity for uncertain and positive words; as these are the result of exploratory analyses, however, they may simply be chance findings.

A final point of interest is that contrasting results were found on the two explicit memory tests used in this study: recall and recognition. On the free recall test, relationships between worry, cognitive avoidance, and physical threat were found. In recognition, however, sensitivity for uncertain and positive words were related to measures of state anxiety, irritability, and social desirability. As already noted, free recall can be seen as a more difficult test of memory because it requires retrieval from memory without the aid of cues. Recognition, in contrast, is an easier test of memory, as the stimuli are provided, and must simply be distinguished from distractors. In this study, therefore, the tendency to worry and engage in cognitive avoidance appeared to contribute to the ability to effortfully retrieve words related to physical threat from memory. As both worry and cognitive avoidance are closely related to anxiety, an 
enhanced memory for threat would be consistent with information processing theory. In contrast, the recognition sensitivity results suggest that mood state and social desirability either facilitated (state anxiety) or hampered (state irritability, social desirability) judgments about whether words were new or old. As high sensitivity scores indicate a better ability to discriminate stimuli from distractors, the results of this study suggest that mood state and social desirability have an impact on this discrimination. Mild levels of state anxiety may facilitate this type of judgment due to heightened arousal and attentiveness. Potentially, as already noted, social desirability may bias unsure participants toward responses that they believe are expected, thereby reducing their accuracy.

While these points are interesting, the results of this study should be interpreted with some limitations in mind. One limitation concerns the sample, which was relatively small, and largely comprised of undergraduate Psychology students. Psychology students may have suspected that the study concerned memory, and have employed memorization strategies. As there was no post-study questioning about the experiment, this could not be assessed. A further concern is that beyond a self-report of English fluency, there was no independent measure of language to verify that participants were fluent English speakers; this may have affected participants' understanding of, and memory for, the stimulus words.

In conclusion, hypotheses concerning intolerance of uncertainty and memory for uncertain and threat words were not supported in Study 2. While there was some evidence that worry was significantly related to memory for threat, this relationship became non-significant when cognitive avoidance was taken into consideration. In 
addition, there was no significant relationship between worry and memory for uncertain words. However, measures of state anxiety did appear to be positively related to memory outcomes (sensitivity for uncertain, threat, and positive words). Conversely, state irritability and social desirability were negatively related to sensitivity for some stimuli (uncertain and positive words). Therefore, in this study, mood state and social desirability appeared to be better predictors of memory for stimulus words than intolerance of uncertainty.

Follow up analyses indicated that three particular forms of cognitive avoidancedistraction, avoidance of threatening stimuli, and thought suppression —-were related to recall, for physical threat words alone. This relationship between cognitive avoidance and memory for threat may be due to a paradoxical increase in the processing of material the individual is trying to avoid. While not the central focus of this study, this is an interesting outcome that will be further explored.

It could be speculated that the lack of a relationship between worry, IU, and memory bias is consistent with the Williams et al (1997) prediction that anxiety is not characterized by an explicit memory bias for threat. It would be unreasonable to draw this conclusion on the basis of non-significant findings from a single study; however, this question will be further pursued in Study 3. 


\section{STUDY 3}

\section{Introduction}

The goal of Study 3 is to investigate the role of IU in implicit memory for threat and uncertainty. More specifically, the Williams and colleagues (1997) prediction of an implicit, perceptual memory bias for threat will be tested. Using the stimuli generated in Study 1, memory for uncertain, threatening, neutral, and positive words will be assessed. Additional variables of interest such as worry, cognitive avoidance, mood, and social desirability will also be explored. In this study, participants will perform an incidental learning task that promotes perceptual processing of the stimulus words (letter counting). They will then complete a tachistoscopic word identification test to assess their implicit perceptual memory. In order to maintain transfer-appropriateness, the task and test will be procedurally identical; moreover, both task and test will require perceptual processing. Rapid presentation times and distractor tasks will be used to prevent accidental elaborative processing of stimuli. In light of the predictions made by Williams et al., and the findings of previous studies, IU is expected to be a significant predictor of an implicit perceptual memory bias for threat and uncertain words. In order to contrast this with any explicit memory that may occur, a free recall test will also be administered. As the task in this study requires implicit, perceptual processing, and because the Williams et al. model does not predict an explicit memory bias, no explicit memory bias is expected in Study 3 .

A new variable that may contribute to memory bias will be introduced in Study 3: that of stimulus relevance. Stimulus relevance refers to the degree to which stimulus words relate to topics that may be currently salient to a participant. For example, while a word such as geographical may be neutral for most people, it could be highly relevant to 
someone about to move to another continent. It is conceivable that highly relevant stimuli would be preferentially processed, and therefore better remembered. In some cases, personal stimulus relevance might be a better predictor of memory bias than the construct the word is intended to represent (i.e., neutral, uncertain, threat, positive). The contribution of stimulus relevance was addressed in a study by Coles et al. (2007; see Information Processing in GAD and Worry for detailed review). In this study, idiosyncratic stimulus words were selected based on participant ratings of personal relevance. However, the personal relevance of the stimulus words was inferred from participant ratings of valence (i.e., rate words on a scale ranging from extremely negative for me to extremely positive for me). While this provided a way to measure the relevance of positive and threat words, it could not as clearly establish the personal relevance of neutral or uncertain words - words that are neither negative nor positive, but may be highly salient. In order to address the influence of relevance on memory, therefore, a direct measure of stimulus relevance was added to Study 3. Specifically, at the end of the experiment, participants were asked to rate all stimulus words on their relevance: ...rate how much each word corresponds to events or topics that are currently important to you. These ratings were then analyzed to assess their contribution to memory. A secondary hypothesis of this study was therefore that stimulus relevance would make a significant contribution to memory for all types of words.

Further measures were added in Study 3 in order to address questions that arose from the results of Study 2. In Study 2, there was no measure of English language fluency beyond self-report; accordingly, a short vocabulary scale was added in Study 3. Vocabulary is of particular concern when participants are encoding complex and abstract 
stimuli such as uncertain words. In addition, a scale measuring the ability to control attention was used, to assess the influence of this ability on the tachistoscopic identification task. A self-report manipulation check was also administered, to evaluate whether participants had discovered the true purpose of the study, and whether they had employed any memorization strategies. Finally, a semi-structured clinical interview (the Mini-International Neuropsychiatric Interview) was administered to ensure that exclusionary criteria were not met.

Given that most findings of implicit memory bias have emerged from studies using clinical samples of GAD patients, the original goal of Study 3 was to approximate these samples by recruiting extreme groups of high and low worriers. Despite our efforts, however, examination of the resulting sample showed normally distributed data, with a mean worry score characteristic of non-clinical student samples (Penn State Worry Questionnaire: $M=51.07, S D=14.70$ ). For this reason, and in order to prevent the loss of power inherent in creating groups from continuous data, worry and all other study variables were analyzed continuously to determine their contribution to memory outcomes.

\section{Method}

\section{Participants}

Participants for this study were recruited through Concordia University's Psychology Department Participant Pool, and received bonus course credit for participation (a bonus 1\% of their final grade per hour of participation). Participants were also recruited from the Montreal community using posters and advertisements on the internet and in a newspaper; these individuals were compensated \$25. All methods of 
recruitment were approved by Concordia University's Human Research Ethics Committee. In all, 423 people contacted our laboratory concerning the study, with 151 dropping out after initial contact. A telephone screening (see Appendix W) was administered to the remaining 272 people to determine eligibility for the study. Participants were eligible for the study if: a) they were between 18-65 years of age; b) English was their first language; c) they had normal or corrected to normal vision and hearing; d) they had never been diagnosed with a reading disability or speech impediment; e) any psychotropic medication had been stable for at least four weeks; f) they did not meet criteria for bipolar disorder, major depression, schizophrenia, substance abuse, or pathological gambling; and g) they had not taken part in associated studies in our laboratory. Participants were also questioned about their levels of worry, in an attempt to acquire equal numbers of high and low worriers for the sample.

Of the 272 screened participants, 128 did not meet study requirements and were excluded; 13 who were eligible dropped out after the screening. A total of 131 participants completed the study. Of these, 5 were used to pilot test the procedure, and 6 were removed due to procedural problems. In order to eliminate a floor effect on the tachistoscopic identification task (the study's main outcome), 22 participants who had extremely low scores (less than 8/64 correct responses) were removed from the sample.

The final sample consisted of 98 individuals, of whom 58 received $\$ 25$ compensation, and 40 received course credit for their participation. The sample included 76 women and 22 men ranging in age from 18 to 64 , with a mean age of $26.23(\mathrm{SD}=$ 10.37). Ethnicity/race was primarily White (68\%), followed by Black (8\%), Middle Eastern (7\%), Asian (5\%), Multi-racial (4\%), Latino/a (3\%), and First Nations (1\%); the 
remainder did not report their ethnicity/race. Eighty-four percent of the sample were students, $70 \%$ of whom were full time; $50 \%$ of the students reported that they were majoring in Psychology. Participants' highest level of completed education was as follows: $44 \%$ had completed CEGEP, $34 \%$ had an undergraduate degree, $21 \%$ had completed high school, and 1\% had completed primary school. Forty-four percent of the sample reported that they did not work, $40 \%$ reported working part-time, and $16 \%$ worked full-time. According to a cut score of 53 on the Penn State Worry Questionnaire, $49 \%$ of the sample were low worriers, and $51 \%$ were high worriers (based on data from Brown, Antony, and Barlow, 1992, a high worry cut score of 53 was established using the method suggested by Jacobson and Truax, 1991). However, despite attempts to recruit distinct high and low worry groups, the participants most resemble a normally distributed student sample (see Table 13 for Study 3 measure means).

\section{Materials}

The stimulus words created in Study 1 were used in this study. Sixty-four of the stimuli were divided into two non-overlapping sets of 32 words- Set A and Set B. Sets A and B included eight of each word type (neutral, uncertain, threatening, and positive), with no word appearing in both sets. Set A and Set B threat words were each comprised of four social threat and four physical threat words. Sets A and B therefore contained the same number of words of each type (see Figure 3 for Set A \& B words). Each of the nonuncertain words was matched on length and part of speech with one corresponding uncertain word (see Table 1 for matching of words). As previously described, equivalent familiarity has already been shown across word categories. Each participant viewed either Set A or Set B during Task 1. Task 2 employed a separate set of Neutral words that 
did not appear in any other task. During Task 3, participants viewed the entire set of 64 words (Set A + Set B). Thus, half of the participants were primed with Set A words during Task 1, and the other half were primed with Set B during Task 1. During the course of the study, incoming participants were randomly assigned to either Group A (priming with Set A words) or Group B (priming with Set B words) in order to control for potential word set effects. Forty-nine participants were randomized into Group A, and 49 into Group B.

\section{Procedure}

Following the telephone screening described above, eligible participants were invited to our laboratory at Concordia University for individual testing. Participants read and signed a consent form before taking part in the study. The consent form stated that the study concerned the relationship between personality and how individuals process words; participants were informed that they would complete tasks related to attention, perception, and word comprehension. If participants had expected a test of memory, they would have been likely to use memorization strategies, making a test of implicit memory impossible. Participants were therefore not informed that their memory would be tested, but were fully debriefed about the true purpose of the research at the end of the study. In total, participants spent approximately ninety minutes in our laboratory. Thirty minutes each were required for the computer tasks, the pencil and paper tasks, and the clinical interview; participants were offered breaks between each of these phases of the study. 
Figure 3

Stimulus Words used in Study 3, Divided into Set A and Set B

Set A Words

\begin{tabular}{|c|c|}
\hline Word & Type \\
\hline ACTUAL & Neutral \\
\hline CUSTOMARY & Neutral \\
\hline EVERY & Neutral \\
\hline EXTERIOR & Neutral \\
\hline GEOGRAPHICAL & Neutral \\
\hline MODERN & Neutral \\
\hline THEORETICAL & Neutral \\
\hline UNIVERSAL & Neutral \\
\hline AMBIGUOUS & Uncertain \\
\hline DEBATABLE & Uncertain \\
\hline MAYBE & Uncertain \\
\hline MYSTERIOUS & Uncertain \\
\hline PUZZLING & Uncertain \\
\hline QUESTIONABLE & Uncertain \\
\hline RANDOM & Uncertain \\
\hline TRICKY & Uncertain \\
\hline CONTAMINATED & Threat-p \\
\hline INCOMPETENT & Threat-s \\
\hline INFERIOR & Threat-s \\
\hline LETHAL & Threat-p \\
\hline MOCKED & Threat-s \\
\hline MUTILATED & Threat-p \\
\hline RUDE & Threat-s \\
\hline SICKNESS & Threat-p \\
\hline ADORED & Positive \\
\hline CARING & Positive \\
\hline CONFIDENT & Positive \\
\hline DELIGHTFUL & Positive \\
\hline ENTHUSIASTIC & Positive \\
\hline GOOD & Positive \\
\hline TAEASANT & Positive \\
\hline & Positive \\
\hline
\end{tabular}

Set B Words

\begin{tabular}{|c|c|}
\hline Word & Type \\
\hline APPARENT & Neutral \\
\hline ENTIRE & Neutral \\
\hline INGREDIENT & Neutral \\
\hline LITERAL & Neutral \\
\hline METHODICAL & Neutral \\
\hline RECIPROCAL & Neutral \\
\hline RURAL & Neutral \\
\hline UNLISTED & Neutral \\
\hline CHANCE & Uncertain \\
\hline DOUBTFUL & Uncertain \\
\hline HESITATION & Uncertain \\
\hline IMPRECISE & Uncertain \\
\hline IRREGULAR & Uncertain \\
\hline UNCERTAIN & Uncertain \\
\hline VAGUE & Uncertain \\
\hline VARYING & Uncertain \\
\hline DISAPPROVAL & Threat-s \\
\hline EPIDEMIC & Threat-p \\
\hline FEVERISH & Threat-p \\
\hline HATED & Threat-s \\
\hline IGNORED & Threat-s \\
\hline RIDICULED & Threat-s \\
\hline UNSANITARY & Threat-p \\
\hline VIRUS & Threat-p \\
\hline ADMIRED & Positive \\
\hline GENEROUS & Positive \\
\hline GREAT & Positive \\
\hline INTEGRITY & Positive \\
\hline OPTIMISTIC & Positive \\
\hline UNSELFISH & Positive \\
\hline WONDERFUL & Positive \\
\hline WORTHY & Positive \\
\hline
\end{tabular}

Note. Threat-p = physical threat words; Threat-s = social threat words. Social threat words are italicized. 
Computer tasks. For the computer tasks, participants were seated $50 \mathrm{~cm}$ away from an IBM-compatible computer with a 17-inch cathode ray tube (CRT) monitor. The monitor had a $16.67 \mathrm{~ms}$ screen refresh rate, and all presentation durations were programmed in accordance with this rate. All computer tasks were conducted using Eprime (version 1.2) software. Throughout the computer tasks, participants heard prerecorded instructions through speakers to the left and right of the computer screen, and were able to adjust the volume to their preference. Instructions were presented auditorily in order to prevent visual priming to the instructions. Participants initiated each new task by pressing the " 1 " on the number keypad, and could replay instructions by pressing " 2 ". Once initiated, tasks could not be paused or stopped. Participants verbally responded to the computer tasks, and their responses were recorded with an audio tape cassette recorder. A research assistant introduced and started each of the computer tasks, and remained in the testing room during Task 2. However, participants carried out Task 1 and Task 3 alone in the testing room. A white noise machine was placed outside the testing room to minimize any distracting noise.

Task 1: Letter counting. The purpose of this task was to ensure that participants focussed on the visual features of each stimulus word. For this task, eight words of each type (neutral, uncertain, threatening, and positive) appeared on the screen, one at a time. Participants therefore viewed a total of 32 words during Task 1, with half of the participants viewing Set A, and the other half viewing Set B. All stimuli were presented in uppercase white Arial font, bolded, size 22, centered on a black screen. Each stimulus word was preceded by a fixation cross $(+)$ which appeared in the middle of the screen for $1000 \mathrm{~ms}$; participants were asked to focus on this cross (note: for ease of reporting, the 
durations are rounded up to the nearest whole number). Following the fixation cross, the stimulus word appeared on the screen for $900 \mathrm{~ms}$. Pilot testing indicated that $900 \mathrm{~ms}$ was long enough to allow perception of the word, while being short enough to maintain the appearance that processing of words was being tested. While the stimulus word was still on the screen, participants heard a randomly selected letter through the computer speakers. Participants were asked to verbally state, as quickly as possible, how many times the letter appeared in the word they saw. The word was then replaced by a mask for $1067 \mathrm{~ms}$ in order to eliminate any visual after-image; this mask was made up of 12 pound signs (\#\#\#\#\#\#\#\#\#\#\#), as the longest stimulus word was 12 letters. For visual clarity, the mask was presented in white Courier New font, size 22. The mask was followed by a blank screen for $1050 \mathrm{~ms}$. This sequence was repeated for each of the 32 words, with the words and letters randomly selected by the computer. A practice trial using 10 neutral words was used to train participants in this procedure, also with the aid of pre-recorded instructions. Once participants had completed the practice trial, they initiated Task 1 with a key press, which then proceeded without further instructions. The neutral words used in the practice trial did not appear in any other task or practice trial.

Task 2: Calibration. The purpose of this task was to calibrate-i.e., determine the optimal presentation speed for each participant_-for Task 3, the tachistoscopic word identification. The goal was to select a duration most likely to result in the correct identification of between $30-50 \%$ of words. Tarsia et al. (2003) recommended a projected rate of $30-50 \%$ identification on a similar tachistoscopic identification task, in order to prevent floor and ceiling effects. During calibration, stimuli were presented in the same 
format as Task 1, with each word preceded by a fixation cross (1000 ms), followed by a mask (1067 ms), and then a blank screen (1050 ms).

Participants first viewed a set of seven neutral words, one at a time, with each word remaining on the screen for $83 \mathrm{~ms}$. They were asked to read each word out loud as it appeared, as quickly as possible. A research assistant remained in the room (giving the rationale that Task 2 was very short), and, unknown to the participant, checked their responses against a list of correct answers. A word had to be read exactly as presented in order to be marked as correct. Using this procedure, participants then viewed three more sets of 7 neutral words, with each set appearing at a shorter duration $(67 \mathrm{~ms}$, $50 \mathrm{~ms}$, and $33 \mathrm{~ms}$, respectively). The pre-recorded instructions informed participants that each successive set would be presented more briefly. No word appeared more than once, either within or across sets. With the aid of a calibration sheet, the research assistant then calculated the presentation duration that would be used during Task 3 . This calculation was based on the participant's worst performance (the duration at which they correctly identified the fewest words). If the participant's worst performance was $4 / 7$ or more ( $>$ $57 \%$ correct), they were assigned one duration faster for Task 3 . For example, a participant who correctly identified $4 / 7$ words at $50 \mathrm{~ms}$ would be assigned to the $33 \mathrm{~ms}$ duration for Task 3 . If the subject's worst performance was $2 / 7$ or $3 / 7$ ( $28 \%-42 \%$ correct), they were assigned to this same duration for Task 3 . If the subject's worst performance was $1 / 7$ or less $(<14 \%$ correct) they were assigned to one duration slower for Task 3. Although participants were not tested at $100 \mathrm{~ms}$ or $17 \mathrm{~ms}$, these durations were used for Task 3 if performance was less than $1 / 7$ at $83 \mathrm{~ms}$ (100 ms used) or more than 4/7 at $33 \mathrm{~ms}$ (17 ms used). Due to the scoring procedure, the word sets in Task 2 
appeared in the same order for all participants. There was no practice trial for Task 2 , and the neutral words used did not appear in any other aspect of the research (see Appendix X for calibration sheet, which shows the neutral words used).

Task 3: Tachistoscopic word identification. This procedure was adapted from Tarsia et al. (2003), and was used to test implicit memory for the words viewed in Task 1 (primed words) and a matched set of novel words (unprimed distractor words). As in Task 2, participants were asked to read aloud words presented briefly on the screen. For Task 3, however, the research assistant initiated the computer program and left the room. Participants then completed the task alone, and their responses were recorded by audio tape cassette. As in previous tasks, each word was preceded by a fixation cross (1000 $\mathrm{ms})$, followed by a mask (1067 ms), and then a blank screen (1050 ms). In Task 3 , however, the presentation duration for the words was the one selected during calibration (100 ms, $83 \mathrm{~ms}, 67 \mathrm{~ms}, 50 \mathrm{~ms}, 33 \mathrm{~ms}$, or $17 \mathrm{~ms})$. As already noted, half of the participants had viewed Set A words at Task 1, and the other half had viewed Set B. All participants viewed the combined set of 64 words during Task 3 (Set A + Set B). In this way, the word set viewed during Task 1 were the primed words, and the set not viewed provided a matched set of unprimed words (see Figure 3 for Task 3 stimuli). Each participant viewed the 64 words one at a time, in a different random order generated by the computer. Because the procedure resembled that of Task 2, and in order to avoid a practice effect, no practice trial was provided. However, participants were cautioned, in both pre-recorded and verbal instructions, that this task would be very challenging due to the short presentation durations; they were encouraged to make a response to every presented word, even if they felt like they were guessing. 
Pencil and paper tasks. Pencil and paper tasks were completed in the same testing room, at a separate desk. Instructions for these tasks were provided verbally by the research assistant, who then left the room to allow participants to complete each of the tasks alone. Note that participants who requested a break at this point in the experiment were asked to wait until the distractor and free recall tasks were complete.

Distractor task. Immediately after the computer tasks, participants completed a distractor task to minimize rehearsal and recency effects. Participants were given a lined sheet with a number (841) in the top right corner. They were asked to subtract three from this number, and then subtract three from the result; they were instructed to continue subtracting by threes until the research assistant returned (after five minutes). They were asked to work as quickly and accurately as possible (see Appendix Y for distractor task).

Free recall task. After the distractor task, participants were given a blank sheet of paper and asked to write down as many words as they could remember, in any order, from any of the computer tasks. The research assistant left the room for five minutes.

Completion of self-report questionnaires. Participants were then asked to complete a package of questionnaires, which were in pseudo-randomized order (4 different orders were used). However, all packages presented the SUDS ratings on the first page, in order to obtain mood ratings immediately after the tasks described above. In addition, the Manipulation Check and Stimulus Relevance questionnaires always appeared at the end of the package to avoid revealing the true purpose of the study, which might have influenced responses to other measures. Participants had unlimited time to complete the questionnaires, but were instructed to make their ratings quickly, without 
spending too long on any one question. Most participants took 30 minutes to complete the questionnaires.

Clinical interview. The final phase of the study was the administration of a semistructured clinical interview, the MINI-International Neuropsychiatric Interview (see Measures). This interview was used to provide corroboration of exclusionary criteria (i.e., no bipolar disorder, major depression, schizophrenia, substance abuse, or pathological gambling). Originally, this interview was also intended to explore the potential impact of other anxiety disorders on memory; however, the non-clinical nature of the sample rendered this impracticable. A trained research assistant and the first author conducted these interviews. Participants were offered a break prior to the interview, which lasted approximately 30 minutes.

Debriefing. Following the interview, participants were fully debriefed about the true purpose of the study. They also received a written debriefing sheet (see Appendix Z) which summarized the goals of the research. Participants were then compensated for their participation ( $\$ 25$ or 2 bonus course credits).

\section{Measures}

The Intolerance of Uncertainty Scale (IUS), the Cognitive Avoidance Questionnaire (CAQ), the Social Desirability Scale (SDS), the Penn State Worry Questionnaire (PSWQ), the Worry and Anxiety Questionnaire (WAQ), the State-Trait Anxiety Inventory-Trait version (STAI-T), the Center for Epidemiologic Studies Depression Scale (CES-D), the Subjective Units of Distress Scale (SUDS), and the General Information Sheet are described in the Study 2 Measures section. 
The Attentional Control Scale (ACS; Derryberry \& Reed, 2001) is a self-report questionnaire measuring the ability to effortfully control one's attention. This measure was included in order to assess the impact of attentional control on the computer tasks in this study. The ACS includes sub factors measuring the ability to focus attention, shift attention between tasks, and flexibly control thought (Derryberry \& Reed, 2002). The ACS is comprised of 20 items, rated on a 4-point Likert scale ranging from $1=$ almost never to $4=$ almost always. The ACS demonstrates good internal consistency, $\alpha=.88$, convergent validity with measures of positive emotionality, and divergent validity with regards to measures of negative emotionality (Derryberry \& Reed, 2002).

The Mini-International Neuropsychiatric Interview (MINI, English version 5.0.0, for DSM-IV; Sheehan et al., 1998) is a brief structured clinical interview that assesses the major Axis I disorders according to DSM and ICD criteria. It is designed for use by psychiatrists and general practitioners, and takes approximately 20 minutes to administer. The MINI is structured in a yes/no response format, evaluating anxiety disorders, mood disorders, eating disorders, substance abuse, and psychotic disorders. The original version of the MINI has shown good validity when compared to other interviews such as the Structured Clinical Interview for DSM-III-R (Sheehan et al, 1997), and the Composite International Diagnostic Interview for ICD-10 (Lecrubier et al, 1997). The version of the MINI used in this study (5.0) has been updated by the authors to correspond with criteria in the DSM-IV and the ICD-10. The MINI is widely employed in clinical research, and has been translated into several languages. (Note: given the length of this interview, the reader is referred to this URL for the full instrument: http://www.chammp.org/LinkClick.aspx?fileticket=E_xTcEd-Glo\%3D\&tabid=210 ). 
The Stimulus Relevance Questionnaire (SRQ) is a custom made self-report measure of the personal relevance of the stimulus words used in this study. This questionnaire was created in order to determine whether higher relevance would be related to better memory for the stimuli. The SRQ lists all 64 stimulus words in random order, and asks respondents to rate how personally relevant each word is; i.e., how much each word corresponds to important events or topics over the past month. Ratings are made on a 5-point Likert scale ranging from $0=$ not at all relevant to $m e$ to $4=$ very relevant to me (see Appendix AA).

A Manipulation Check (MC) form was created in order to assess participants' awareness of the true purpose of the study. On the MC, respondents were asked to choose the answer they feel best describes the purpose of the computer tasks (i.e., the implicit memory test). Six alternative answers are presented in multiple choice format, including five distractors (e.g., To assess the speed of your perceptual ability when working with words; Don't know/none of the above); and only one correct response (To measure memory for different words). Respondents are then asked to briefly explain their choice in a space at the bottom of the questionnaire (see Appendix AB).

The Mill Hill Vocabulary Scale (Mill Hill Vocabulary Scale Junior, Multiple Choice, Set A) is a companion form to Raven's Progressive Matrices and Vocabulary Scales (Raven, Court, \& Raven, 1977). Together, the Raven scales are designed to measure verbal knowledge and ability. The current study used only a self-report version of the vocabulary scale, the Mill Hill Vocabulary Scale-Multiple Choice (MHVS- MC), to provide a brief measure of English language comprehension, and assess its impact on responses to word stimuli. The MHVS-MC has 33 items, in which a word is provided, 
and respondents are asked to select the correct synonym from a list of six alternatives. While there are norms for the MHVS, these apply to the full version (i.e., not the multiple choice version used in this study, which is different in form and content). However, Mathews et al. (1995) and Mogg et al. (1987) also used the MHVS-MC as a measure of vocabulary. Due to copyright restrictions, this measure cannot be reproduced. The reader is referred to Pearson Assessments and Information (Psychcorp): http://www.pearsonassessments.com/pai/, where a search for Mill Hill Vocabulary will produce the full Raven's Progressive Matrices and Vocabulary Scales; the MHVS-MC is the fifth section of this measure.

\section{Results}

\section{Data Screening and Outlier Analyses}

All data were double-entered, compared, and corrected to ensure accuracy. To screen the data for univariate outliers, a $z$-score was computed for all study variables, and any score greater than \pm 3.29 was considered an outlier (Tabachnik \& Fidell, 2001). According to this criterion, several participants were univariate outliers on age; as this is a participant characteristic, it was not transformed. To screen for multivariate outliers, the variables were entered into a linear regression, with Mahalanobis and Cook's distances computed; because age was a univariate outlier, it was not included. Mahalanobis values showed that two participants had scores greater than the Chi-square critical value, $\chi^{2}(16)$ $=26.30, p<.05$, and were therefore multivariate outliers. However, neither of these outliers showed Cook's distances greater than 1, indicating they would not have a significant effect on a regression analysis (Tabachnik \& Fidell, 2001), and they were retained. 
To evaluate skewness, $z$-scores were computed by dividing skewness by its standard error for all variables; any value greater than 2.58 was considered significantly skewed (Field, 2005). Using this criterion, several Subjective Units of Distress (SUDS) scores were significantly skewed: SUDS sadness (3.71) and irritability (3.02) were positively skewed, and SUDS well-being (-3.09) was negatively skewed. Scores on the IUS were also positively skewed (2.97). This pattern on both SUDS ratings and the IUS would be expected in a non-clinical sample. Furthermore, IUS and SUDS ratings were only involved in regression analyses, which do not require normality of predictors (Field, 2005, p. 170); therefore, these variables were not transformed. Means and standard deviations for Study 3 measures are presented in Table 13, and correlations in Table 14.

\section{Manipulation Checks}

The Manipulation Check questionnaire (MC). Participants' awareness of the purpose of the computer tasks was assessed using the self-report MC; a total of 79 participants completed this multiple-choice measure. There are six possible answers on the MC, but results showed that one item was not chosen by any participants (i.e., that the purpose of the computer tasks was "To measure your verbal ability"); this item was therefore removed from the analyses, leaving only five remaining items. It was expected that one-fifth of the participants would choose the correct answer by chance (i.e., that the purpose of the computer tasks was "To measure memory for different words"). The remaining four-fifths of the participants were expected to choose any one of the other distractor items. Fifty-seven participants chose one of the distractor items, compared to 22 who chose the correct answer. A Chi-square test showed that the proportion of participants choosing the correct answer was not significantly higher than would be 
expected by chance, $\chi^{2}(1, n=79)=3.04, p=.08$. In addition, participants wrote a brief explanation of the response they selected; a review of these showed that even participants who chose the correct answer were not aware of the true purpose of the study. 
Table 13

Means and Standard Deviations for Study 3 Measures

\begin{tabular}{|c|c|c|c|}
\hline Measure & $n$ & $M$ & $S D$ \\
\hline Attentional Control Scale & 97 & 52.30 & 9.06 \\
\hline Center for Epidemiologic Studies Depression Scale & 98 & 14.48 & 8.61 \\
\hline Cognitive Avoidance Questionnaire & 98 & 59.40 & 18.88 \\
\hline CAQ Avoidance & 98 & 11.58 & 5.41 \\
\hline CAQ Distraction & 98 & 13.10 & 4.81 \\
\hline CAQ Substitution & 98 & 9.44 & 3.60 \\
\hline CAQ Suppression & 98 & 14.76 & 4.75 \\
\hline CAQ Transformation & 98 & 10.49 & 4.58 \\
\hline Intolerance of Uncertainty Scale & 98 & 61.80 & 20.33 \\
\hline IUS Factor 1 & 98 & 31.12 & 11.57 \\
\hline IUS Factor 2 & 98 & 30.68 & 10.37 \\
\hline Mill Hill Vocabulary Scale & 98 & 25.91 & 3.09 \\
\hline Penn State Worry Questionnaire & 98 & 51.07 & 14.70 \\
\hline Social Desirability Scale & 98 & 5.93 & 3.03 \\
\hline State-Trait Anxiety Inventory-Trait version & 97 & 42.73 & 11.66 \\
\hline SUDS Anxiety & 98 & 42.74 & 26.09 \\
\hline SUDS Fatigue & 98 & 43.81 & 25.43 \\
\hline SUDS Irritability & 98 & 27.74 & 25.12 \\
\hline SUDS Sadness & 98 & 19.56 & 20.58 \\
\hline SUDS Well-being & 98 & 70.54 & 18.22 \\
\hline Worry and Anxiety Questionnaire & 98 & 36.66 & 17.07 \\
\hline
\end{tabular}

Note. $\mathrm{CAQ}=$ Cognitive Avoidance Questionnaire: Avoidance $=$ Avoidance of threatening stimuli subscale; Distraction = Distraction subscale; Substitution $=$ Thought substitution subscale; Suppression $=$ Thought suppression subscale; Transformation $=$ Transformation of images into thoughts subscale. IUS = Intolerance of Uncertainty Scale. SUDS = Subjective Units of Distress Scale 
Table 14

Correlations Between Study 3 Measures

\begin{tabular}{|c|c|c|c|c|c|c|c|c|c|c|c|c|c|}
\hline ACS & CAQ & CES & IUS & PSW & SDS & STA & SUa & SUf & SUi & SUs & SUw & Voc V & WAQ \\
\hline ACS & $-.32^{* *}$ & $-.48^{* *}$ & $-.39^{* *}$ & -.46 & $.35^{* *}$ & $-.40^{* *}$ & -.10 & -.12 & -.14 & -.17 & $.22^{*}$ & -.00 & $-.46^{* *}$ \\
\hline CAQ & & $.55^{* *}$ & $.62^{* *}$ & $.65^{* *}$ & -.18 & $.59^{* *}$ & $.44^{* *}$ & .11 & $.30^{* *}$ & $.37^{* *}$ & $-.24^{*}$ & -.04 & $.60^{* *}$ \\
\hline CES & & & $.60^{* *}$ & $.57^{* *}$ & $-.24^{*}$ & $.71^{* *}$ & $.34^{* *}$ & $.24^{*}$ & $.31^{* *}$ & $.57^{* *}$ & $-.48^{* *}$ & .07 & $.67^{* *}$ \\
\hline IUS & & & & $.76^{* *}$ & $-.24^{*}$ & $.80^{* *}$ & $.37^{*}$ & $.20^{*}$ & $.32^{* *}$ & $.42^{* *}$ & $-.39^{* *}$ & .14 & $.76^{* *}$ \\
\hline PSW & & & & & $-.22^{*}$ & $.77^{* *}$ & $.56^{* *}$ & $.26^{* *}$ & $.46^{* *}$ & $.48^{* *}$ & $-.42^{* *}$ & .01 & $.82^{* *}$ \\
\hline SDS & & & & & & $-.24^{*}$ & -.04 & .03 & -.14 & -.02 & .06 & $-.36^{* *}$ & $-.29^{* *}$ \\
\hline STA & & & & & & & $.46^{* *}$ & $.25^{*}$ & $.45^{* *}$ & $.51^{* *}$ & $-.53^{* *}$ & .13 & $.86^{* *}$ \\
\hline SUa & & & & & & & & .08 & $.53^{* *}$ & $.48^{* *}$ & -.19 & -.05 & $.48^{* *}$ \\
\hline SUf & & & & & & & & & $.27^{* *}$ & $.27^{* *}$ & $-.28^{* *}$ & -.01 & $.25^{*}$ \\
\hline SUi & & & & & & & & & & $.49^{* *}$ & -.13 & -.05 & $.44^{* *}$ \\
\hline SUs & & & & & & & & & & & $-.29^{* *}$ & -.09 & $.42^{* *}$ \\
\hline SUw & & & & & & & & & & & & -.14 & $-.58^{* *}$ \\
\hline Voc & & & & & & & & & & & & & .13 \\
\hline WAQ & & & & & & & & & & & & & \\
\hline
\end{tabular}

Note. $n=89$. ACS = Attentional Control Scale; $\mathrm{CAQ}=$ Cognitive Avoidance Questionnaire; $\mathrm{CES}=$ Center for Epidemiologic Studies Depression scale; IUS = Intolerance of Uncertainty Scale; PSW = Penn State Worry Questionnaire; SDS = Social Desirability Scale; STA = State-Trait Anxiety Inventory-Trait version; $\mathrm{SU}=$ Subjective Units of Distress: $\mathrm{a}=$ anxiety, $\mathrm{f}=$ fatigue, $\mathrm{i}=$ irritability, $\mathrm{s}=$ sadness, $\mathrm{w}=$ wellbeing; Voc $=$ Mill Hill Vocabulary Scale; WAQ $=$ Worry and Anxiety Questionnaire. ${ }^{*} p<.05 .{ }^{* *} p<.01$. 
Priming effect. As previously noted, participants were primed with one of two sets of words during Task 1: Set A or Set B. The combined Sets A + B provided a test of primed and unprimed words during Task 3, tachistoscopic word identification. Based on the words correctly identified during Task 3 , a priming score was calculated. The priming score was the difference between correctly identified primed and unprimed words, such that: priming score $=$ \# correct primed words - \# correct unprimed words. This difference was calculated in order to control for each individual's baseline level of word identification. In addition to a total score, priming scores were also calculated for each of the four word types; for example: priming on neutral words = \# correct primed neutral words - \# correct unprimed neutral words. Note that because half of the participants were primed with Set A, and the other half with Set B, the calculations differed for Group A and B: Group A priming = \# correct primed words from Set A - \# correct unprimed words from Set B; and Group B priming = \# correct primed words from Set B - \# correct unprimed words from Set $A$.

In order to establish whether scores were higher overall for primed as compared to unprimed words (i.e., whether there was a priming effect), $t$-tests were performed separately for Group A and B. In Group A, the total score on primed (Set A) words ( $M=$ $22.43, S E=.72)$ was significantly higher than the total score on unprimed (Set B) words $(M=17.53, S E=.94),, t(96)=4.13, p<.001)$. In Group B, the total score on primed (Set B) words $(M=18.37, S E=1.11)$ was significantly higher than the total score on unprimed (Set A) words $(M=13.27, S E=.93),, t(96)=-3.52, p<.001)$. Therefore, a significant priming effect was present in both groups. 
Word set effect. Further analyses suggested that despite careful matching, Set A and Set B words were associated with different levels of priming. A $t$-test showed a significantly higher total priming score in Group A $(M=9.16, S E=.56)$ compared to Group B $(M=.84, S E=.53), t(96)=10.81, p<.001)$. Additional analyses were conducted to determine whether there was a significant priming effect when neutral, uncertain, threat, and positive words were examined separately. To this end, a series of paired-samples $t$-tests were conducted; again, Groups A and B were analyzed separately. For neutral, uncertain, threat, and positive words, results showed a significant priming effect in Group A (see Table 15). In Group B, there was a significant priming effect for threat and positive words. However, priming was non-significant for uncertain words in Group B. In addition, a reverse priming effect was found for neutral words in Group B, such that identification of unprimed neutral words was significantly higher than primed words (see Table 16). As this will affect analyses involving uncertain and neutral word priming, selected analyses were conducted using Group A alone, because Group A showed a significant priming effect across all word types (see Intolerance of Uncertainty, Stimulus Relevance, and Priming).

Word frequencies. In order to determine whether any individual words showed a floor or ceiling effect, frequencies of correct identification on the word identification task were examined for each of the 64 words. Frequencies showed that there were no words with a floor effect (i.e., no words with zero correct identifications); there were also no words with a ceiling effect (i.e., no words that all participants correctly identified). Further examination showed that the two words with the lowest identification rate were "contaminated" (13\% correct) and "ridiculed" (15\% correct). Conversely, the two words 
Table 15

Study 3: Paired Samples t-tests of Priming on Neutral, Uncertain, Threat and Positive Words in Group $A$

\begin{tabular}{llllll}
\hline Word type pairs & $M^{a}$ & $S E$ & $S E M^{b}$ & $t^{c}$ \\
\hline
\end{tabular}

\section{Neutral words}

Set A

Set B

\section{Uncertain words}

Set A

Set B

\section{Threat words}

Set A

Set B

\section{Positive words}

Set A

Set B
6.04

.22

2.78

.27

$3.33 \quad .23$

.23

$.2513 .04 * *$

7

.23

4.08

.26

.20

$9.43 * *$

$2.20 \quad .30$

.30

6.31

.17

$.245 .66^{* *}$

4.96

.28

Note. $n=49 ; d f=48$ for all analyses.

${ }^{a}$ Mean number of correctly identified words on the word identification task.

${ }^{\mathrm{b}}$ Standard error of the mean of paired differences.

${ }^{\mathrm{c}} \mathrm{A}$ significant $t$ value indicates a significant priming effect: the number of correctly identified primed (Set A) words is higher than the number of correctly identified unprimed (Set B) words.

$* * p<.001$. 
Table 16

Study 3: Paired Samples t-tests of Priming on Neutral, Uncertain, Threat and Positive Words in Group B

\begin{tabular}{lllll}
\hline Word type pairs & $M^{a}$ & $S E$ & $S E M^{b}$ & $t^{c}$ \\
\hline
\end{tabular}

\section{Neutral words}

Set A

Set B

Uncertain words

Set A

Set B

Threat words

Set A

Set B

\section{Positive words}

Set A

Set B
4.92

.26

.25

$2.21 *$

4.37

.32

4.45

.28

.23

$-.44$

$4.55 \quad .28$

3.02

.25

$.22-3.79 * *$

3.84

.35

5.14

.28

$.17 \quad-2.80 *$

5.61

.28

Note. $n=49 ; d f=48$ for all analyses.

${ }^{a}$ Mean number of correctly identified words on the word identification task.

${ }^{\mathrm{b}}$ Standard error of the mean of paired differences.

${ }^{\mathrm{c}} \mathrm{A}$ significant negative $t$ value indicates a significant priming effect: the number of correctly identified primed (Set B) words is higher than the number of correctly identified unprimed (Set A) words. A significant positive t value indicates a reverse priming effect, with unprimed (Set A) words significantly higher than primed (Set B) words.

$* p<.05 . * * p<.001$. 
with the highest identification rate were "confident" (94\% correct) and "wonderful" (95\% correct). The words with the lowest identification rate were both from the subset of threat words, with “contaminated" being part of Set A, and "ridiculed" part of Set B. The words with the highest identification rate were both from the subset of positive words, with "confident" being part of word Set A, and "wonderful" word Set B. Given that these words did not display absolute floor or ceiling effects, and because they were equally distributed between Set A and Set B, they were all retained for analysis.

\section{Regressions With All Study Variables}

As in the previous study, diagnostic indices were calculated (as per Field, 2005), and showed that the necessary assumptions for regression analyses were met. In order to explore the contribution of all the study variables to priming scores, four separate regressions were used to predict priming on each type of word (neutral, uncertain, threat, and positive). Scores on study measures except the Intolerance of Uncertainty Scale (IUS) and the Stimulus Relevance Questionnaire (SRQ) were entered in one step as predictors. Although previous analyses showed a non-significant priming effect in Group B for neutral and uncertain words, the regressions with all study variables were conducted with the whole sample, because using Group A alone would have reduced the sample size considerably $(n=49)$, problematic in analyses with 18 predictors. Moreover, when responses from Groups A and B are pooled, a significant overall priming effect is present.

Results of these analyses showed that group was a significant negative predictor of priming for neutral words $(\beta=-3.56, p<0.001)$, uncertain words $(\beta=-2.57, p<$ $0.001)$, threat words $(\beta=-1.19, p<0.001)$, and positive words $(\beta=-.89, p=0.011)$. 
Essentially, being in Group A and being primed with Set A words, was a significant predictor of priming. Female sex was also a significant predictor of priming on neutral words $(\beta=-0.21, p=0.009)$, as was cognitive avoidance $(\beta=0.21, p=0.048)$.

Vocabulary was a significant predictor of priming on uncertain words $(\beta=0.31, p=$ $0.004)$, and cognitive avoidance was a predictor of priming on threat words $(\beta=0.35, p=$ $0.03)$.

Another four regressions were then conducted to identify variables contributing to recall on each of the four word types; again, measures except the IUS and SRQ were entered in one step as predictors. Results showed that age was a significant negative predictor of neutral word recall $(\beta=-0.26, p=0.043)$. Cognitive avoidance was a negative predictor of recall for uncertain words $(\beta=-0.39, p=0.017)$. Duration of the word identification task was a significant predictor of recall for threat words $(\beta=0.27, p$ $=0.017)$, and SUDS fatigue ratings were negatively related to threat word recall $(\beta=-$ $0.32, p=0.008)$. For recall of positive words, trait anxiety was a significant predictor ( $\beta$ $=0.54, p=0.036)$, and depression was a negative predictor $(\beta=-0.48, p=0.031)$.

Given these results, age, sex, and vocabulary were entered in the first steps of subsequent regressions. The significant relationship between group and priming reflects the previous finding that Group A had significantly higher priming scores than Group B. This group effect has already been noted, and appears to be due to differences between the two word sets (see Word set effect). However, this group effect is not related to the central study hypotheses, which concern the relationship between intolerance of uncertainty, stimulus relevance, and memory. Therefore, to avoid replicating already established group/word set differences, and to maintain statistical power for analysis of 
the study hypotheses, group was not included as a predictor in further regressions. Findings concerning cognitive avoidance, threat word priming, and uncertain word recall were examined in follow up analyses. While other reported findings are potentially interesting, they were not explored because they were less directly related to the study hypotheses. Moreover, given the study's small sample size, it was a priority to limit both the number of analyses, and the number of predictors within each analysis.

\section{Intolerance of Uncertainty, Stimulus Relevance, and Priming}

Regressions were then used to address hypotheses concerning the role of intolerance of uncertainty and stimulus relevance in implicit memory (priming). To this end, four separate hierarchical regressions were conducted, with priming on each of the four word types (neutral, uncertain, threat, and positive) as the outcome. Because age, sex, and vocabulary were significant predictors in previous regressions, these were entered in Step 1. Scores on the Intolerance of Uncertainty Scale (IUS) were entered in Step 2, to determine whether IU made a significant contribution to priming beyond these variables. Stimulus Relevance (SRQ) scores were totaled separately for each word type, and were entered in Step 3 to determine whether stimulus relevance added to the prediction of priming scores, beyond age, sex, vocabulary, and intolerance of uncertainty.

Results showed that for neutral words, female sex was a significant predictor of priming in all steps of the model, but no other variables were significant (see Table 17). Given that priming was non-significant for neutral words in Group B (see Word set effect), this regression was re-run using Group A alone. Results showed that in Group A, only age was a significant negative predictor of neutral word priming, in Step 1 ( $\beta=$ $0.34, p=0.04)$. 
Table 17

Study 3: Hierarchical Multiple Regression with Demographics, Intolerance of

Uncertainty, and Stimulus Relevance Predicting Priming on Neutral Words

\begin{tabular}{|c|c|c|c|c|c|}
\hline Predictor & $\Delta R^{2}$ & $B$ & $S E B$ & $\beta$ & $95 \% \mathrm{CI}$ \\
\hline Step 1 & $.09 *$ & & & & \\
\hline Constant & & -.61 & 2.21 & & {$[-4.99,3.78]$} \\
\hline Age & & -.05 & .03 & -.20 & {$[-.10, .00]$} \\
\hline Sex & & -1.43 & .63 & $-.23 *$ & {$[-2.69,-.17]$} \\
\hline Vocab & & .14 & .09 & .17 & {$[-.04, .31]$} \\
\hline
\end{tabular}

Step 2

.01

Constant

Age

Sex

Vocab

IUS

Step 3

Constant

Age

Sex

Vocab

IUS

SRn
$-1.09$

$-.05$

$-1.32$

.12

.02
2.24

.03

.64

.09

.01
[-5.54, 3.36]

$-.20$

$[-.10, .00]$

$-.21 *$

$[-2.59,-.05]$

.14

$[-.06, .30]$

.12

$[-.01, .04]$

.00

$\begin{array}{rrcc}-1.16 & 2.33 & & {[-5.78,3.47]} \\ -.05 & .03 & -.20 & {[-.10, .00]} \\ -1.33 & .65 & -.22 * & {[-2.62,-.04]} \\ .12 & .09 & .14 & {[-.06, .30]} \\ .02 & .01 & .12 & {[-.01, .04]} \\ .00 & .02 & .01 & {[-.04, .05]}\end{array}$

Note. $n=98 . \mathrm{CI}=$ confidence interval for $B$. Vocab $=$ Mill Hill Vocabulary Scale; IUS = Intolerance of Uncertainty Scale; $\mathrm{SRn}=$ stimulus relevance for neutral words.

$* p<.05$. 
For uncertain words, female sex and vocabulary were significant predictors of priming in Step 1; when the IUS was entered in Step 2, only vocabulary was a significant predictor. In Step 3, sex, vocabulary, and the stimulus relevance of uncertain words were all significant predictors of uncertain word priming (see Table 18). As priming was nonsignificant for uncertain words in Group B , this regression was re-run with Group A alone. Results showed that in Group A, vocabulary was the only significant predictor of uncertain word priming, in all steps of the regression (Step 3: $\beta=0.46, p=0.007$ ).

For threat and positive words, neither age, sex, vocabulary, intolerance of uncertainty, nor stimulus relevance were significant predictors of priming (see Tables 19 $\& 20)$.

\section{Intolerance of Uncertainty, Stimulus Relevance, and Recall}

A parallel set of regressions were then carried out to examine the contribution of intolerance of uncertainty and stimulus relevance to recall for the stimuli. Again, four separate hierarchical regressions were conducted, with recall for neutral, uncertain, threat, and positive words as separate outcomes. Age, sex, and vocabulary were entered in Step 1; intolerance of uncertainty in Step 2; and stimulus relevance for each type of word in Step 3.

Results showed that neither age, sex, vocabulary, intolerance of uncertainty, nor stimulus relevance predicted recall for any type of word, in any step of the regressions (see Appendices AC - AF). 
Table 18

Study 3: Hierarchical Multiple Regression with Demographics, Intolerance of

Uncertainty, and Stimulus Relevance Predicting Priming on Uncertain Words

\begin{tabular}{|c|c|c|c|c|c|}
\hline Predictor & $\Delta R^{2}$ & $B$ & $S E B$ & $\beta$ & $95 \% \mathrm{CI}$ \\
\hline Step 1 & $.09 *$ & & & & \\
\hline Constant & & -3.29 & 1.76 & & {$[-6.79, .21]$} \\
\hline Age & & -.01 & .02 & -.06 & {$[-.05, .03]$} \\
\hline Sex & & -1.01 & .51 & $-.20 *$ & {$[-2.01,-.00]$} \\
\hline Vocab & & .20 & .07 & $.30 *$ & {$[.06, .34]$} \\
\hline
\end{tabular}

Step 2

.00

Constant

Age

Sex

Vocab

IUS

Step 3

Constant

Age

Sex

Vocab

IUS

SRun
$-4.32$

.00

$-1.23$

.21

$-.02$

.05
1.80

.02

.51

.07

.01

.00

20

$.06^{*}$

$-.01$

$-1.01$

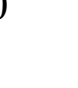


Table 19

Study 3: Hierarchical Multiple Regression with Demographics, Intolerance of

Uncertainty, and Stimulus Relevance Predicting Priming on Threat Words

\begin{tabular}{|c|c|c|c|c|c|}
\hline Predictor & $\Delta R^{2}$ & $B$ & $S E B$ & $\beta$ & $95 \% \mathrm{CI}$ \\
\hline Step 1 & .02 & & & & \\
\hline Constant & & -.60 & 1.36 & & {$[-3.31,2.11]$} \\
\hline Age & & .00 & .02 & .02 & {$[-.03, .04]$} \\
\hline Sex & & -.14 & .39 & -.04 & {$[-.92, .64]$} \\
\hline Vocab & & .07 & .06 & .15 & {$[-.04, .18]$} \\
\hline
\end{tabular}

Step 2

.00

Constant

Age

Sex

Vocab

IUS

Step 3

Constant

Age

Sex

Vocab

IUS

SRt
$-.63$

.00

$-.14$

.07

.00
1.39

.02

.40

.06

.01

.01

$$
-.69
$$

.01

$-.10$

.07

.01

$-.02$
1.40

.02

.40

.06

.01

.02
[-3.39, 2.14]

.02

$[-.03, .04]$

$[-.93, .65]$

$[-.04, .18]$

.14

.01

$[-.02, .02]$

Note. $n=98 . \mathrm{CI}=$ confidence interval for $B$. Vocab $=$ Mill Hill Vocabulary Scale; IUS = Intolerance of Uncertainty Scale; $\mathrm{SRt}=$ stimulus relevance for threat words. 
Table 20

Study 3: Hierarchical Multiple Regression with Demographics, Intolerance of

Uncertainty, and Stimulus Relevance Predicting Priming on Positive Words

\begin{tabular}{lccccc}
\hline Predictor & $\Delta R^{2}$ & $B$ & $S E B$ & $\beta$ & $95 \%$ CI \\
\hline Step 1 & .01 & & & & \\
Constant & & 1.46 & 1.33 & & {$[-1.19,4.12]$} \\
Age & -.01 & .02 & -.09 & {$[-.04, .02]$} \\
Sex & .30 & .38 & .08 & {$[-.46,1.06]$} \\
Vocab & -.01 & .05 & -.02 & {$[-.12, .10]$}
\end{tabular}

Step 2

Constant

Age

Sex

Vocab

IUS .00

$1.44 \quad 1.36$

$-.01$

.30

.02

$-.09$

$[-.04, .02]$

.39

.08

$[-.47,1.07]$

$-.01$

.06

$-.02$

$[-.12, .10]$

.00

.01

.01

$[-.02, .02]$

Step 3

.00

Constant

2.04

1.68

[-1.30, 5.38]

Age

$-.01$

.02

$-.08$

$[-.04, .02]$

Sex

.31

.39

.09

[-.46, 1.09]

Vocab

$-.02$

.06

$-.04$

$[-.13, .09]$

IUS

.00

.01

$-.01$

$[-.02, .02]$

SRpos

$-.01$

.01

$-.07$

$[-.04, .02]$

Note. $n=98 . \mathrm{CI}=$ confidence interval for $B$. Vocab $=$ Mill Hill Vocabulary Scale; IUS = Intolerance of Uncertainty Scale; SRpos = stimulus relevance for positive words. 


\section{Follow up Analyses: Cognitive Avoidance}

Given that cognitive avoidance emerged as a significant predictor of threat word priming, and a significant negative predictor of uncertain word recall, a series of follow up analyses were used to explore these results.

Cognitive avoidance and threat word priming. With threat word priming scores as the outcome, a hierarchical regression was conducted with age, sex, and vocabulary in Step 1; cognitive avoidance in Step 2; and stimulus relevance for threat words in Step 3. Results showed that the only significant predictor of threat word priming was cognitive avoidance, in Steps 2 and 3 (Step 3: $\beta=0.30, p=0.009)$.

As previously outlined, the Cognitive Avoidance Questionnaire (CAQ) can also be analyzed in terms of its five subscales: thought substitution; transformation of images into thoughts; distraction; avoidance of threatening stimuli; and thought suppression. Given the significant findings with the CAQ total score, another set of regressions was carried out, each using a different CAQ subscale as a predictor. Specifically, age, sex, and vocabulary were entered in Step 1; one of the CAQ subscales in Step 2; and stimulus relevance for threat words in Step 3. Separate regressions were conducted for each of the CAQ subscales in order to avoid problems with collinearity.

Results showed that CAQ thought substitution was a significant predictor of threat word priming, in Steps 2 and 3 of the regression. CAQ avoidance of threatening stimuli was also a significant predictor in Steps 2 and 3; in addition, stimulus relevance was a negative predictor in Step 3. CAQ thought suppression was also a significant predictor of threat word priming, in Steps 2 and 3. There were no significant findings for CAQ transformation of images into thoughts, or CAQ distraction (see Tables 21 - 25). 
Table 21

Study 3: Hierarchical Multiple Regression with Demographics, Cognitive Avoidance (Thought Substitution Subscale), and Stimulus Relevance Predicting Priming on Threat Words

\begin{tabular}{lccccc}
\hline Predictor & \multicolumn{1}{c}{$B R^{2}$} & $B$ & $S E B$ & $\beta$ & $95 \%$ CI \\
\hline Step 1 & .02 & & & & \\
Constant & & .60 & 1.36 & & {$[-3.31,2.11]$} \\
Age & .00 & .02 & .02 & {$[-.03, .04]$} \\
Sex & -.14 & .39 & -.04 & {$[-.92, .64]$} \\
Vocab & .07 & .06 & .15 & {$[-.04, .18]$} \\
& & & & \\
Step 2 & $.06 *$ & & & & {$[-4.17,1.30]$} \\
Constant & & -1.43 & 1.38 & $.02, .04]$ \\
Age & .01 & .02 & .06 & {$[-.95, .57]$} \\
Sex & & .19 & .38 & -.05 & {$[-.04, .17]$} \\
Vocab & & .06 & .05 & .13 & {$[.02, .19]$} \\
CAQsub & & .10 & .04 & $.24 *$ &
\end{tabular}

Step 3

Constant

Age

Sex

Vocab

CAQsub

SRt

.03

$\begin{array}{rrcc}-1.51 & 1.37 & & {[-4.22,1.21]} \\ .01 & .02 & .08 & {[-.02, .04]} \\ -.19 & .38 & -.05 & {[-.94, .56]} \\ .07 & .05 & .13 & {[-.04, .17]} \\ .13 & .05 & .30^{*} & {[.04, .22]} \\ -.02 & .02 & -.17 & {[-.05, .01]}\end{array}$

Note. $n=98 . \mathrm{CI}=$ confidence interval for $B$. Vocab $=$ Mill Hill Vocabulary Scale; CAQsub = Cognitive Avoidance Questionnaire, Thought substitution subscale; SRt = stimulus relevance for threat words. $* p<.05$. 
Table 22

Study 3: Hierarchical Multiple Regression with Demographics, Cognitive Avoidance (Transformation of Images into Thoughts Subscale), and Stimulus Relevance Predicting Priming on Threat Words

\begin{tabular}{|c|c|c|c|c|c|}
\hline Predictor & $\Delta R^{2}$ & $B$ & $S E B$ & $\beta$ & $95 \% \mathrm{CI}$ \\
\hline Step 1 & .02 & & & & \\
\hline Constant & & -.60 & 1.36 & & {$[-3.31,2.11]$} \\
\hline Age & & .00 & .02 & .02 & {$[-.03, .04]$} \\
\hline Sex & & -.14 & .39 & -.04 & {$[-.92, .64]$} \\
\hline Vocab & & .07 & .06 & .15 & {$[-.04, .18]$} \\
\hline Step 2 & .01 & & & & \\
\hline Constant & & -.94 & 1.41 & & {$[-3.75,1.86]$} \\
\hline Age & & .00 & .02 & .03 & {$[-.03, .04]$} \\
\hline Sex & & -.09 & .40 & -.03 & {$[-.87, .70]$} \\
\hline Vocab & & .07 & .06 & .14 & {$[-.04, .18]$} \\
\hline CAQtra & & .03 & .04 & .10 & {$[-.04, .10]$} \\
\hline Step 3 & .01 & & & & \\
\hline Constant & & -1.02 & 1.41 & & {$[-3.82,1.79]$} \\
\hline Age & & .01 & .02 & .04 & {$[-.03, .04]$} \\
\hline Sex & & -.06 & .40 & -.02 & {$[-.84, .73]$} \\
\hline Vocab & & .08 & .06 & .15 & {$[-.03, .18]$} \\
\hline CAQtra & & .05 & .04 & .14 & {$[-.03, .12]$} \\
\hline SRt & & -.02 & .02 & -.13 & {$[-.05, .01]$} \\
\hline
\end{tabular}

Note. $n=98 . \mathrm{CI}=$ confidence interval for $B$. Vocab $=$ Mill Hill Vocabulary Scale; CAQtra $=$ Cognitive Avoidance Questionnaire, Transformation of images into thoughts subscale; $\mathrm{SRt}=$ stimulus relevance for threat words. 
Table 23

Study 3: Hierarchical Multiple Regression with Demographics, Cognitive Avoidance (Distraction Subscale), and Stimulus Relevance Predicting Priming on Threat Words

\begin{tabular}{|c|c|c|c|c|c|}
\hline Predictor & $\Delta R^{2}$ & $B$ & $S E B$ & $\beta$ & $95 \% \mathrm{CI}$ \\
\hline Step 1 & .02 & & & & \\
\hline Constant & & -.60 & 1.36 & & {$[-3.31,2.11]$} \\
\hline Age & & .00 & .02 & .02 & {$[-.03, .04]$} \\
\hline Sex & & -.14 & .39 & -.04 & {$[-.92, .64]$} \\
\hline Vocab & & .07 & .06 & .15 & {$[-.04, .18]$} \\
\hline
\end{tabular}

.00

Step 2

Constant

Age

Sex

Vocab

CAQdis
$-.89$

.00

$-.17$

.08

.02
1.48

.02

.40

.06

.03

.01

Step 3

Constant

Age

Sex

Vocab

CAQdis

SRt
$-1.04$

.01

$-.19$

.08

.03

$-.02$
1.48

.02

.40

.06

.04

.02
$[-3.83,2.04]$

.03

$-.05$

.15

.06

$[-.96, .62]$

$[-.03, .18]$

$[-.05, .08]$

Note. $n=98 . \mathrm{CI}=$ confidence interval for $B$. Vocab $=$ Mill Hill Vocabulary Scale; CAQdis $=$ Cognitive Avoidance Questionnaire, Distraction subscale; SRt $=$ stimulus relevance for threat words. 
Table 24

Study 3: Hierarchical Multiple Regression with Demographics, Cognitive Avoidance (Avoidance of Threatening Stimuli Subscale), and Stimulus Relevance Predicting Priming on Threat Words

\begin{tabular}{|c|c|c|c|c|c|}
\hline Predictor & $\Delta R^{2}$ & $B$ & $S E B$ & $\beta$ & $95 \% \mathrm{CI}$ \\
\hline Step 1 & .02 & & & & \\
\hline Constant & & -.60 & 1.36 & & {$[-3.31,2.11]$} \\
\hline Age & & .00 & .02 & .02 & {$[-.03, .04]$} \\
\hline Sex & & -.14 & .39 & -.04 & {$[-.92, .64]$} \\
\hline Vocab & & .07 & .06 & .15 & {$[-.04, .18]$} \\
\hline Step 2 & $.05 *$ & & & & \\
\hline Constant & & -1.50 & 1.39 & & {$[-4.26,1.27]$} \\
\hline Age & & .00 & .02 & .00 & {$[-.03, .03]$} \\
\hline Sex & & -.10 & .38 & -.03 & {$[-.86, .66]$} \\
\hline Vocab & & .08 & .05 & .16 & {$[-.03, .19]$} \\
\hline CAQav & & .07 & .03 & $.23 *$ & {$[.01, .12]$} \\
\hline
\end{tabular}

Step 3

$.04 *$

Constant

Age

Sex

Vocab

CAQav

SRt
$-1.78$

.00

$-.07$

.09

.10

$-.03$
1.37

.02

.38

.05

.03

.02
$[-4.51, .95]$

$[-.03, .03]$

$[-.82, .68]$

$[-.01, .20]$

$[.03, .16]$

$[-.06,-.00]$

Note. $n=98 . \mathrm{CI}=$ confidence interval for $B$. Vocab $=$ Mill Hill Vocabulary Scale; CAQav = Cognitive Avoidance Questionnaire, Avoidance of threatening stimuli subscale; $\mathrm{SRt}=$ stimulus relevance for threat words. $* p<.05$. 
Table 25

Study 3: Hierarchical Multiple Regression with Demographics, Cognitive Avoidance (Thought Suppression Subscale), and Stimulus Relevance Predicting Priming on Threat Words

\begin{tabular}{|c|c|c|c|c|c|}
\hline Predictor & $\Delta R^{2}$ & $B$ & $S E B$ & $\beta$ & $95 \% \mathrm{CI}$ \\
\hline Step 1 & .02 & & & & \\
\hline Constant & & -.60 & 1.36 & & {$[-3.31,2.11]$} \\
\hline Age & & .00 & .02 & .02 & {$[-.03, .04]$} \\
\hline Sex & & -.14 & .39 & -.04 & {$[-.92, .64]$} \\
\hline Vocab & & .07 & .06 & .15 & {$[-.04, .18]$} \\
\hline Step 2 & $.05 *$ & & & & \\
\hline Constant & & -1.75 & 1.42 & & {$[-4.58,1.07]$} \\
\hline Age & & .00 & .02 & .02 & {$[-.03, .03]$} \\
\hline Sex & & -.14 & .38 & -.04 & {$[-.90, .62]$} \\
\hline Vocab & & .08 & .05 & .15 & {$[-.03, .18]$} \\
\hline CAQsupp & & .08 & .03 & $.23^{*}$ & {$[.01, .14]$} \\
\hline
\end{tabular}

Step 3

.02

$\begin{array}{lrrcc}\text { Constant } & -1.89 & 1.42 & & {[-4.70, .93]} \\ \text { Age } & .00 & .02 & .03 & {[-.03, .04]} \\ \text { Sex } & -.13 & .38 & -.04 & {[-.89, .62]} \\ \text { Vocab } & .08 & .05 & .16 & {[-.03, .19]} \\ \text { CAQsupp } & .09 & .03 & .28^{*} & {[.02, .16]} \\ \text { SRt } & -.02 & .02 & -.16 & {[-.05, .01]}\end{array}$

Note. $n=98 . \mathrm{CI}=$ confidence interval for $B$. Vocab = Mill Hill Vocabulary Scale; CAQsupp = Cognitive Avoidance Questionnaire, Thought suppression subscale; SRt = stimulus relevance for threat words. $* p<.05$. 
Follow-up analyses were then conducted to explore differences between social threat words and physical threat words, with regards to the three CAQ subscales that were significant predictors of threat word priming. Specifically, to examine priming on social threat words, a regression was conducted with age, sex, and vocabulary entered in Step 1; one of the three CAQ subscales in Step 2; and stimulus relevance for social threat words in Step 3. A parallel procedure was then followed for priming of physical threat words, but with stimulus relevance for physical threat words in Step 3.

For social threat words, results indicated that none of these variables were significant predictors of priming. For priming of physical threat words, CAQ thought substitution was a significant predictor at Steps 2 and 3 of the model (Step 3: $\beta=0.29, p$ $=0.004)$; vocabulary was also a predictor at Step $1(\beta=0.22, p=0.048)$. CAQ avoidance of threatening stimuli was a significant predictor of physical threat word priming at Steps 2 and 3 (Step 3: $\beta=0.36, p=0.001$ ); vocabulary was significant at all steps (Step 3: $\beta=$ $0.24, p=0.022$ ); and stimulus relevance for physical threat words was a negative predictor at Step $3(\beta=-0.25, p=0.015)$. CAQ thought suppression was a significant predictor of physical threat word priming at Step $3(\beta=0.22, p=0.036)$, and vocabulary was a significant predictor at all steps (Step 3: $\beta=0.22, p=0.043)$.

Cognitive avoidance and uncertain word recall. Finally, to explore the relationship between cognitive avoidance and uncertain word recall, a hierarchical regression was conducted with age, sex, and vocabulary in Step 1; the total CAQ score in Step 2; and stimulus relevance for uncertain words in Step 3. The results showed that stimulus relevance for uncertain words was the only significant predictor of uncertain word recall $(\beta=0.27, p=0.031)$. 


\section{Discussion}

This final study explored the contribution of intolerance of uncertainty (IU) and stimulus relevance to implicit memory for different types of words. Participants performed an incidental learning task, and then completed measures of implicit and explicit memory. As the task was designed to promote implicit memory, no explicit biases were predicted. However, IU was expected to be significantly related to implicit memory for threatening and uncertain words; stimulus relevance was also expected to contribute. The impact of factors such as vocabulary, attentional control, worry, cognitive avoidance, and depression were also explored.

Manipulation checks showed that the computer tasks produced a significant overall priming effect. However, there was a word set effect, such that priming was higher among Group A participants, who were exposed to Set A words during the first task. Analyses were carried out as planned, but follow up analyses using Group A—who showed significant priming for all word types - were also conducted. Responses to the Manipulation Check questionnaire indicated that participants were unaware of the true purpose of the computer tasks.

Exploratory regressions with all study variables showed that some participantrelated variables (i.e., age, sex, vocabulary) were related to both priming and recall for stimulus words. However, these analyses also showed that worry was not significantly related to priming or recall for the stimulus words; similarly, no other symptom measures were significant predictors. Conversely, factors such as stimulus relevance, and processes such as cognitive avoidance, did appear to be related to memory outcomes. 
The first study hypothesis, that intolerance of uncertainty would predict implicit memory (i.e., priming scores) for uncertain and threat words, was not supported. The secondary hypothesis, that stimulus relevance would make a contribution to implicit memory, was supported, but for uncertain words only. Other variables that contributed to priming included female sex (for neutral and uncertain words) and vocabulary (for uncertain words only).

A parallel set of analyses showed no relationship between intolerance of uncertainty and recall for any type of word; worry, stimulus relevance and other variables were similarly unrelated. As the goal of the procedure was to produce implicit memory, the lack of relationship between study variables and explicit memory is unsurprising. In terms of IU, these findings are similar to those of Study 2, in which IU was not significantly related to explicit memory bias. Conversely, in preliminary Study 2 analyses, worry was significantly related to explicit memory for threat, which was not the case in Study 3. It bears repeating, however, that the encoding task in Study 3 was not intended to create explicit memory.

While there were no specific hypotheses concerning cognitive avoidance, follow up analyses suggested that it was a significant predictor of implicit memory for threat words. Further exploration suggested that specific forms of cognitive avoidancethought substitution, avoidance of threatening stimuli, and thought suppression-were related to physical threat word priming. Vocabulary was also a significant predictor of physical threat word priming. Stimulus relevance, in contrast, was a negative predictor of threat priming, but only in conjunction with avoidance of threatening stimuli. The results concerning cognitive avoidance echo the findings from Study 2, in which distraction, 
avoidance of threatening stimuli, and thought suppression were significant predictors of recall for physical threat words. In both studies, significant relationships between cognitive avoidance and memory only emerged for physical threat words. Furthermore, avoidance of threatening stimuli and thought suppression were significant predictors in both Study 2 and 3 (thought substitution was only significant in Study 3). This recurrence suggests that the relationship between these types of cognitive avoidance and memory for physical threat may not be a chance finding. As noted in Study 2, memory may be specific to physical rather than social threat because of the high concreteness and imageability of physical threat words, or because the construct of physical threat is more salient, or differently encoded. What is notably different between the two studies, however, is the type of memory in question: in Study 2, this effect was found for free recall; in Study 3, for perceptual priming. This seeming discrepancy may simply reflect the different types of encoding required by the tasks in each study. For example, in Study 2 , words were projected on a screen and rated on familiarity; encoding was explicit, as was the free recall test. In Study 3, encoding was intentionally perceptual, as was the tachistoscopic test of implicit memory. While the results of Studies 2 and 3 concern different forms of memory (conceptual and perceptual), therefore, they may in fact reflect a similar process that is occurring with regards to the encoding of physical threat.

Although stimulus relevance was expected to contribute to memory for all types of words, it only reliably emerged as a negative predictor of threat word priming (in the CAQ avoidance of threatening stimuli analyses). This might indicate that some form of avoidance, or directing of processing away from threat, occurs when threat is personally relevant. As the relationship between stimulus relevance and priming was negative, in 
this case it appeared that the attempt to avoid thinking about threat was successful. In addition, this relationship appeared to be specific to physical threat. Although one analysis suggested that stimulus relevance was a significant predictor of priming on uncertain words, this relationship no longer held when analyses were repeated with the group demonstrating a priming effect on uncertain words (Group A). Alternatively, it may be that the scant findings with regards to stimulus relevance are due to flaws in the method of measurement. For example, certain types of words may be more difficult to rate in terms of their self-relevance. Uncertain words, for example, may as a group be difficult to relate to personal concerns, leading participants to spend more time rating them, and potentially inflating their ratings. Conversely, there may be a reluctance to relate threatening words to current personal concerns, and this may lead to artificially lowered stimulus relevance ratings.

There are naturally limitations to this study, the most evident being the use of a non-clinical student sample to examine questions of memory bias. As previously noted, we attempted to recruit a sample with high worriers, in order to approximate GAD research; however, this was not possible. It may be that the lack of findings concerning IU are due to the non-clinical, moderate levels of worry present in this study's sample. Another consideration is the properties of the stimulus words used in the study. Specifically, results indicate that vocabulary was related to uncertain word priming. This suggests that despite their statistically similar ratings of familiarity (as found in Study 1), uncertain words may have been more complex, and better vocabulary may have allowed for better priming. However, vocabulary was also related to priming of physical threat words in the cognitive avoidance analyses; therefore, better vocabulary may contribute to 
greater ease of priming in general. Another limitation was the finding of a word set effect, which showed that despite the piloting and matching process, the word sets were not equivalent. This suggests that a larger sample could have been used to test the properties of the words, in order to ensure better matching. On a broader level, however, randomized assignment of participants to different word sets, and subsequent comparisons of word set effects, might be used in future research to ensure equivalency.

To summarize, hypotheses concerning intolerance of uncertainty and implicit memory for uncertain and threat words were not supported in this study. Stimulus relevance did contribute to implicit memory, but negatively, and only for threat words. The most interesting finding from this study was that cognitive avoidance was a significant predictor of priming for threat. Specifically, thought substitution, avoidance of threatening stimuli, and thought suppression were related to implicit memory for physical threat words. As these findings echo those from Study 2, the potential relationship between cognitive avoidance and memory bias for threat and will be considered in the General Discussion. 


\section{General Discussion}

The central goal of the current study was to explore the relationship between IU and memory biases for threat and uncertainty. This relationship was examined by analyzing the contribution of IU as a predictor of memory, and by using stimulus words denoting uncertainty. A related interest was to assess the predictions of Williams et al. (1997), that anxiety is characterized by an implicit but not an explicit memory bias for threat. Cognitive avoidance, stimulus relevance, and a range of other factors were explored. Results of the current studies did not support any of the hypotheses concerning IU. Limited support was found for stimulus relevance, which was negatively related to memory for physical threat. However, two forms of cognitive avoidance-avoidance of threatening stimuli and thought suppression — were related to memory for physical threat, across two studies.

In light of previous research, the non-significant findings concerning IU are unexpected. The IU outcomes from this group of studies can be interpreted in several ways. The first possibility is that IU does not play a role in information processing, and more specifically, in memory for threat and uncertainty. Previous research does suggest that attention, and particularly interpretation, are influenced by IU. However, few studies to date have explored these questions, making predictions about the role of IU in information processing, and particularly memory, difficult. In contrast to expectations, therefore, it may be that IU is actually unrelated to memory bias for threat and uncertainty. As will later be debated, other variables such as cognitive avoidance may be more prominently involved in memory for threat and uncertainty. Another possibility is that IU is related to memory for threat and uncertainty, but that these biases were not 
revealed by the current research. Although transfer appropriateness and cutting-edge information processing tasks could be considered strengths of this research, it is nevertheless the first exploration of memory bias and IU. There may well be other means of addressing this question that would establish the role of IU in information-processing; one goal of this discussion will be to suggest some possibilities.

Non-significant findings concerning IU may be due in part to limitations in the word stimuli that were used. Despite careful analysis and selection, the higher concreteness and imageability of physical threat words may have made them more memorable than uncertain words; this could have masked any processing biases toward uncertainty. There may also have been limitations with the method used to establish the construct validity of uncertain stimuli. Specifically, asking participants to rate stimulus words on how much they referred to uncertainty may have failed to fully capture the uncertainty construct. However, uncertain words were significantly higher on the uncertainty dimension compared to other words; moreover, different patterns of responses to uncertain words suggest that they were conceptually distinct from other word types. Nevertheless, a differently worded question, or a different way of rating the words, may have better established the construct validity of the uncertain stimuli. Alternately, the use of words may have been in and of itself a limitation. As previously noted, words were used in the current research due to their effectiveness in attention and interpretation studies, and their adaptability to information processing tasks. However, some researchers have posited that more ecologically valid approaches should be used to study information processing biases (i.e., McNally, 1995). The lack of significant findings concerning IU may therefore be due to the limited capacity of words to elicit 
information processing biases. While finding other means to explore the processing of uncertainty will be challenging, some alternatives will later be proposed.

The current research provided some evidence that stimulus relevance influences memory; however, this influence was negative, and was limited to threat words. This outcome might be due to limitations with the method used to measure stimulus relevance. Another possibility, however, is that the Study 3 findings do reveal a differential effect of stimulus relevance, which depends on the type of stimuli. For example, stimulus relevance may impair memory for threatening stimuli, but enhance memory for nonthreatening stimuli. This might reflect some type of cognitive avoidance, such that the more personally relevant the threat word, the less it is elaborated and remembered. This is interesting to consider in light of other study findings concerning cognitive avoidance. In any event, the fact that stimulus relevance was a better predictor of memory than vocabulary, or even IU, suggests that it is an important factor to consider in future research.

A parallel goal of the current studies was to test the Williams et al. (1997) prediction, that anxious individuals should show an implicit perceptual memory bias for threat, but not an explicit memory bias. At the broadest level, the results of these studies do not support the Williams et al. model, because there was no evidence of a relationship between anxiety (i.e., trait anxiety, state anxiety, or worry) and implicit perceptual memory for threat. The second and related prediction made by the model is the absence of a relationship between anxiety and explicit memory for threat. In Study 3, there was certainly no relationship between any form of anxiety, and explicit memory for threat; however, there were also no implicit memory biases associated with these variables. 
Therefore, little can be inferred about the Williams et al. model from Study 3. There was similarly no support for the predictions of this model in Study 2; in fact, some of the findings contradict their predictions about the absence of explicit memory bias for threat. For example, worry was significantly related to explicit memory for threat (although not when cognitive avoidance was taken into account); moreover, state anxiety was related to explicit memory for threat. While Study 2 does not support the Williams et al.

predictions, it should be noted that the better test of the Williams model is in fact Study 3, as it tests both implicit and explicit memory following a single encoding task. In addition, Study 3 follows the procedure recommended by Williams et al.—a perceptual encoding task, and an implicit perceptual memory test. Together, however, the results of Study 2 and Study 3 do not provide any conclusive evidence in support of the Williams et al. model.

On a related note, it was surprising that trait anxiety was not related to explicit memory for threat in the current studies. While there were no specific hypotheses concerning trait anxiety, previous research suggests a relationship between trait anxiety and memory bias for threat. As a review of the literature shows, however, findings have been mixed (see Information Processing in High Trait Anxiety). Nevertheless, some studies have found a bias for threat in high trait anxiety; moreover, this pattern is conceptually consistent with the Williams et al. (1997) model, and with information processing theories of anxiety. In this light, the current findings concerning trait anxiety merit some consideration. As already noted, levels of trait anxiety in Study 2 were somewhat low (State-Trait Anxiety Inventory—Trait version: $M=40.24, S D=10.38$; range $29.86-50.62$ ) This moderate range of scores may have prevented the detection of 
biases related to high trait anxiety in Study 2. In Study 3, levels of trait anxiety were somewhat higher and closer to the range found in previous studies (State-Trait Anxiety Inventory-Trait version: $M=42.73, S D=11.66$; range $31.07-54.39)$. However, trait anxiety was only related to recall for positive words in Study 3. The lack of an explicit memory bias for threat may have been due in part to the task used in Study 3, which was specifically geared toward implicit rather than explicit memory. As previously discussed, many studies of trait anxiety have focussed on explicit memory for threat; however, of those that explored implicit memory, the majority did not find a memory bias. Interestingly, two previous studies that did find an "implicit" bias (e.g., Eysenck \& Byrne, 1994; Richards \& French, 1991) used a word-stem completion task to measure implicit memory, which, as already noted, can be influenced by explicit memory (Perruchet \& Baveux, 1989). Therefore, the literature provides little indication that an implicit memory bias for threat should be expected among high trait anxious individuals. In this sense, the findings of Study 3 are consistent with the literature. For Study 2, a slightly low range of trait anxiety scores may have precluded detection of a memory bias for threat that might have been found in a highly trait anxious sample.

There may be another explanation for the lack of trait anxiety findings in these studies, however. Some research suggests that high trait anxious individuals have an attentional bias toward threat, but only under conditions of stress. In one study, for example, high and low trait-anxious students responded similarly to the emotional Stroop under normal circumstances; however, when exams approached, the high trait-anxious students became slower at naming threat words, indicating a bias toward threat (MacLeod \& Rutherford, 1992). Several other studies have found speeded identification of probes 
that follow threatening stimuli; once again, however, these biases were only reliably found when high trait anxious individuals were under stress (Bradley, Mogg, \& Millar, 2000; Mogg, Bradley, \& Hallowell 1994). Taken together, this research suggests that high trait anxiety may be related to information processing biases primarily under conditions of stress (MacLeod \& Mathews, 1988). While these studies concerned attention and not memory, it is possible that memory for threat may only be biased when anxious individuals also experience high levels of state anxiety. In Study 2, levels of state anxiety were quite low overall (SUDS anxiety rating out of 100: $M=31.47, S D=24.48$; range $6.99-55.95)$; as noted above, trait anxiety scores were also fairly low. This combination of low levels of state and trait anxiety may have made it difficult to elicit biases toward threat in Study 2. An anxiety induction procedure (such as telling participants they will later give a speech) might have activated processing biases toward threat; this type of induction could be added to future studies. In Study 3, it is interesting to observe that the levels of state anxiety were somewhat higher (SUDS anxiety rating out of 100: $M=$ $42.74, S D=26.09$; range $16.65-68.83$ ). This may reflect the more challenging nature of the tachistoscopic identification task, which may itself have served as a state anxiety induction. As summarized above, levels of trait anxiety were also higher in Study 3. However, there were no significant relationships between implicit memory for threat, state anxiety, and trait anxiety. As noted above, however, existing research does not clearly predict implicit memory biases in high trait anxiety; evidently, further research on this question is needed.

An intriguing series of outcomes in this research was the relationship between cognitive avoidance and memory for threat in Studies 2 and 3. Cognitive avoidance was 
included in these studies because research suggests a link between cognitive avoidance, worry, and IU. In addition, some researchers have posited that cognitive avoidance might interfere with processing of threat among anxious individuals (i.e., Friedman et al., 2000). In the current studies, cognitive avoidance appeared to be a stronger predictor of memory for threat than either worry or IU. Moreover, cognitive avoidance was positively related to memory for threat, contrasting with the expectation that cognitive avoidance would result in poorer memory for threat. More specifically, in Study 2, cognitive avoidance was a significant predictor of recall for threat words, in conjunction with state anxiety. Detailed analyses revealed that three specific forms of cognitive avoidance were related to recall for physical threat: distraction, avoidance of threatening stimuli, and thought suppression. A similar pattern of results emerged in Study 3, but for priming rather than recall. In Study 3, follow up analyses indicated that thought substitution, avoidance of threatening stimuli, and thought suppression were significantly related to priming for physical threat words; vocabulary was also a contributor. As previously noted, the procedures in Study 2 and 3 were designed to create explicit and implicit memory biases, respectively. This is essentially the pattern that emerged for cognitive avoidance in the two studies. The commonality between the studies is, firstly, that cognitive avoidance was a significant predictor of memory for physical threat words. Secondly, in both studies, avoidance of threatening stimuli and thought suppression were significant predictors of memory for threat. The fact that avoidance of threatening stimuli and thought suppression were significant in both studies raises the possibility that these two types of cognitive avoidance are specifically related to enhanced memory for threat. 
One interesting possibility is that cognitive avoidance was related to recall of threat because attempts to avoid thinking about threat were ineffective. The cognitive avoidance questionnaire measures the self-reported tendency to engage in cognitive avoidance, rather than the degree of success in these attempts. Current study findings may even suggest that attempts to avoid thinking about threat paradoxically increase processing, and result in enhanced memory. In both Study 2 and Study 3, processing resources may have involuntarily been allocated toward threat by individuals who, when given the opportunity, would avoid thinking about threat. High scores on a measure such as the Cognitive Avoidance Questionnaire may represent an information processing style characterized by the tendency to effortfully avoid thinking about threat. These cognitively avoidant individuals may initially succeed in suppressing threat-related information, but subsequently experience an increase of those very thoughts; this is analogous to the rebound effects of thought suppression (Wegner, 1994). Across these two studies, therefore, there may be a cognitively avoidant information processing style that paradoxically enhances memory for threatening material.

The proposal that cognitive avoidance can paradoxically lead to enhanced processing of threat is broadly consistent with the literature on thought suppression. In their classic study, Wegner and colleagues found that suppressing thoughts of a white bear resulted in an increase of those thoughts (Wegner, Schneider, Carter, \& White, 1987). To explain this and similar findings, Wegner proposed that two processes occur during thought suppression. The first, the operating process, is thought to be an effortful and conscious attempt to find distracting stimuli to replace the unwanted thought. The second, automatic, monitoring process, is thought to scan for the presence of the 
unwanted thought, and monitor the success of the suppression attempt. While the goal of these processes is to eliminate unwanted thoughts, they actually serve to associate an increasing number of cues with the unwanted thought, paradoxically making it more accessible (Wegner, 1994). Numerous studies have demonstrated the paradoxical effects of thought suppression in non-clinical samples (see Abramowitz, Tolin, \& Street, 2001; Rassin, Merckelbach, and Muris, 2000, for reviews). A review of this research shows that two consequences of thought suppression have been reported in the literature: enhancement (increases in the thought during attempts to suppress it) and rebound (increases in the thought subsequent to suppression). A recent meta-analysis of thought suppression studies shows that while enhancement effects do exist, individuals are fairly successful in their short-term attempts to suppress. In contrast, rebound effects appear to be larger and more robust across studies. Together, these findings suggest that the rebound effects of thought suppression are most evident after, rather than during, suppression attempts (Abramowitz et al., 2001).

In terms of the current research, therefore, it is conceivable that cognitively avoidant individuals attempted to suppress processing of threat words, and later experienced a rebound effect for those words. This rebound effect might have made threat words more accessible later, during the memory tests that were administered at the end of Studies 2 and 3. Some support for this proposal can be drawn from a recent study showing that thought suppression was associated with an explicit memory bias for threat as compared to neutral words (Kircanski, Craske, \& Bjork, 2008). Kircanski et al., however, used a sample of high trait anxious participants, and moreover did not find an 
association between thought suppression and implicit memory, which contrasts with the current Study 3 findings.

To expand on this line of reasoning, the results of Studies 2 and 3 may suggest that cognitive avoidance is not only a predictor of memory bias, but a better predictor of memory bias than anxiety. The lack of consistent findings in the field of memory and anxiety may have emerged because a separate process, such as cognitive avoidance, must interact with anxiety in order to produce a memory bias. For example, a highly anxious individual may show enhanced attention for threat, but only show a memory bias if they also have a cognitively avoidant style that leads them to engage in thought suppression. In this case, all anxious individuals would show an attentional bias for threat, but only those who are also cognitively avoidant would show a memory bias. Interestingly, these ideas broadly parallel the framework of Williams et al. (1997), in the sense that biases in attention may not necessarily imply the type of processing that would result in biased memory. In the scenario proposed above, memory might only result from the enhanced accessibility and elaboration of stimuli that occurs due to the rebound effects of thought suppression. It is noteworthy that many models of GAD currently include cognitive avoidance as an important contributor to worry and anxiety (e.g., Borkovec et al., 1998; Dugas, Marchand, et al., 2005 ). The results of the current studies suggest the possibility that cognitive avoidance is not only be related to anxiety and worry, but to an avoidant information processing style that predicts the development of memory bias for threat. While this is an interesting proposal, it naturally requires further experimental investigation. 


\section{Suggestions for Future Research}

While the research hypotheses received limited support, some suggestions for future research can nevertheless be derived from the current studies. The first set of recommendations concern some of the methodological issues raised in the literature review. In particular, the outcomes of the current research highlight the need for careful selection of word stimuli in information processing research. Study 1, for example, showed successful word matching in accord with standard information processing procedures, as well as good construct validity. However, differences in the imageability and concreteness of uncertain and physical threat words were found. Because of this, research conclusions about findings concerning IU, and cognitive avoidance of physical threat, can only be tentatively made. Given that most studies in this area have not made detailed analyses of word stimuli, these types of characteristics have remained largely uncontrolled and may have influenced research outcomes. Given enough time and resources, the preferred procedure would be to pilot test stimuli in advance of the research, using the population of interest (i.e., students, high trait anxious individuals, patients seeking treatment, etc.). A less time-consuming alternative, however, may be to obtain participant ratings of stimuli following testing, and use these ratings when interpreting results. This is an approach used to good effect in some previous studies (i.e., Becker et al., 1999). In order to reduce the burden on participants, the of ratings could be limited to valence and imageability. While previous research shows that imageability and concreteness can be differentiated, some researchers suggest that concrete words are more memorable because of their higher imageability (Altarriba et al., 1999; Paivio et al., 1968). If this is the case, a rating of imageability might capture the dimension underlying 
the facilitated processing of both highly concrete and imageable words. In addition, if stimuli related to a specific symptom or disorder are of interest, pre-selection of these stimuli by experts would be recommended, with more detailed pilot testing using a small target population. However it is approached, adding some form of stimulus rating to future studies would clarify whether uncontrolled characteristics of stimuli are influencing study outcomes.

A further area for investigation concerns the role of stimulus relevance in information processing. As outlined in the literature review, only one other study to date has specifically examined this question (Coles et al., 2007). In the current research, participants were simply asked to rate each stimulus word on its personal relevance. While the question used to assess stimulus relevance in this study appeared to have good face validity, different means of wording such a question, and less time-consuming ratings via computer presentation, could improve measurement. It might also be useful to pilot test different stimulus relevance questions to determine how participants interpret them; this may be particularly useful when asking participants to rate words, such as uncertain words, that may be less clearly applicable to personal concerns. Furthermore, different means of analysis could be considered. In Study 3, for example, stimulus relevance was averaged across each word type, and this average was then used as a predictor. Future studies might involve analysis of individual stimulus words that are high on relevance, rather than an averaging across words. However this question is approached, stimulus relevance appears to be a pertinent factor to consider in future studies. 
Findings from the current research suggest that cognitive avoidance, and more specifically, avoidance of threatening stimuli and thought suppression, may be associated with memory for threat. Future studies could be carried out to explore this association. For example, incidental learning of different types of words could be administered to participants high and low in trait anxiety; levels of cognitive avoidance could be used as a predictor of memory outcomes. Such a design might reveal whether cognitive avoidance and anxiety interact to produce a memory bias for threat, or whether cognitive avoidance alone is related to a memory bias (i.e., if high cognitive avoidance predicts memory for threat, regardless of levels of trait anxiety). A further extension would be to explore the effect of different types of cognitive avoidance on memory in high and low trait anxious individuals; as in the current study, thought suppression and avoidance of threatening stimuli may emerge as a key forms of avoidance related to memory.

Finally, suggestions can be made concerning future investigations of IU, worry, and memory. If the current group of studies were replicated, some improvements might render them more effective. For example, pilot testing a large pool of uncertain words in a sample of high worriers may produce stimuli more representative of the uncertainty construct. In the same vein, the current studies could be carried out in high worry groups, and clinical samples of GAD patients. While research suggests that worry exists on a continuum (Olatunji et al., 2010; Ruscio et al., 2001), there are nevertheless qualitative differences between clinical and non-clinical worriers (i.e., Ruscio \& Borkovec, 2004), which may affect information processing. More generally, including measures of both attention and memory would enhance future research. Because the goal of the current studies was to explore different forms of memory bias, attention was not measured. 
However, a focus on one aspect of processing is necessarily limited; in the current studies, for example, attentional factors are likely to have influenced memory outcomes. Measurement of several aspects of processing within the same study would therefore provide a more complete picture. An adaptation of the Study 3 procedure might include a Stroop task for encoding, with measurement of colour naming latency as a measure of attention toward threat. This could then be followed by an implicit test of memory (tachistoscopic identification of Stroop and distractor words). A similar approach has been used in several previous studies, some of which have assessed both attention and memory (i.e., MacLeod \& McLaughlin, 1995; Mathews et al., 1995; Mogg et al., 1989). To date, this has resulted in mixed findings, possibly due to some of the procedural issues already discussed; nevertheless, these studies provide a model for the type of procedure that would be beneficial for this area of research. This approach would have the further advantage of providing a more complete test of the Williams et al. (1997) model, which, although not specifically supported by the current study, provides a comprehensive account of much of the research in this area.

Another avenue for future research might be to move away from the use of words altogether. As previously noted, some researchers advocate more ecologically valid means of evaluating information processing biases (i.e., McNally, 1995). The results of the current studies certainly highlight the many factors that can influence participant responses to word stimuli. One alternative to word stimuli might be to ask participants to complete a task which induces uncertainty. For example, participants might view a series of simple shapes on a computer screen. They could be asked to make some type of response to each shape, and be given a keypad which includes several response options 
(for example, keys marked negative; positive; incomplete; complete). Participants could be told that their task is to decide how to respond, without further instructions as to how they should proceed. Individuals high in IU would be expected to experience more anxiety, and less tolerance for uncertainty, during this task; their responses are likely to be slowed compared to those low in IU. If, moreover, the stimuli varied in their clarity (either visual sharpness or identifiability as specific objects), individuals high in IU might be expected to show longer response latencies as stimuli became less clear. A subsequent memory test might show that high IU individuals, when engaged in an uncertain task, have a memory bias for uncertain stimuli. If needed, levels of threat might be manipulated through instructions that either imply there is no right way to respond (low threat), or that there is only one right way to respond (high threat). While this approach would eliminate some of the problems associated with word stimuli, it is limited in the sense that creating stimuli that represent other categories of information (neutral, threatening, positive, etc.) would be difficult. Potentially, using images that depict differently valenced scenes might be used. This is an approach applied by Koerner and Dugas (2007), who used images derived from the International Affective Picture System classified as neutral, positive, threatening, or ambiguous, to explore the role of IU in interpretation. Some of their findings suggested that individuals high in IU made more negative interpretations of negative and ambiguous scenes, compared to individuals low in IU. The type of images used in their study could be adapted to the type of uncertainty manipulation described above. While interesting, this proposal still suffers from a potentially limited relationship to real life topics of concern for participants. The study conducted by Coles and colleagues (2007) suggests one possible solution: a pre-test evaluation, which would 
determine current worry themes for each participant; some representation of these themes could then be used as testing material. Rather than yoking worriers with controls, however, moderate worriers could be yoked to high worriers; in this way, high and moderate worriers could be matched on worry themes, and identical stimuli administered to each yoked pair of participants. With this design, it might be possible to detect differences in attention and memory among worriers lower and higher in IU.

In conclusion, while the results of these studies did not establish the role of IU in memory for threat and uncertainty, they did generate several observations and potential directions for future research. Findings concerning cognitive avoidance in particular may suggest new avenues for exploring memory bias in anxiety. While designing meaningful studies of information processing and IU will remain a challenge, the endeavor is a valuable one. This type of research will serve to clarify the cognitive processes that underlie worry, and inform theoretical models of anxiety disorders such as GAD. Although there is empirical support for models such as that proposed by Dugas and colleagues (Dugas, Gagnon, et al., 1998), the specific mechanisms underlying processes such as IU remain to be elucidated. Information processing frameworks, whether traditional or innovative in their application, have the potential to answer fundamental questions about how processes such as IU operate. Further studies concerning the processes underlying memory are urgently needed; this is underscored by the lack of clarity in existing research, and the debate concerning which conceptual models should be used to guide future research. Hopefully, studies such as this one will serve to enhance future research on the role of processes such as IU in attention, interpretation, and memory. 


\section{References}

Abramowitz, J. S., Tolin, D.F., \& Street, G. P. (2001). Paradoxical effects of thought suppression: A meta-analysis of controlled studies. Clinical Psychology Review, 21, 683-703.

Altarriba, J., Bauer, L. M., \& Benvenuto, C. (1999). Concreteness, context availability, and imageability ratings and word associations for abstract, concrete, and emotion words. Behavior Research Methods, Instruments, and Computers, 31, 578-602.

American Psychiatric Association. (1980). Diagnostic and statistical manual of mental disorders (3rd ed.). Washington DC: Author.

American Psychiatric Association. (1987). Diagnostic and statistical manual of mental disorders (3rd ed., revised). Washington DC: Author.

American Psychiatric Association. (1994). Diagnostic and statistical manual of mental disorders (4th ed.). Washington DC: Author.

American Psychiatric Association. (2000). Diagnostic and statistical manual of mental disorders (4th ed., text revision). Washington DC: Author.

Andrews, G., Hobbs, M. J., Borkovec, T. D., Beesdo, K., Craske, M. G., Heimberg, R. G., Rapee, R. M., Ruscio, A. M., Stanley, M. A. (2010). Generalized Worry Disorder: A review of DSM-IV Generalized Anxiety Disorder and options for DSM-V. Depression and Anxiety, 27, 134-147.

Bar-Haim,Y., Lamy, D., Pergamin, L., Bakermans-Kranenburg, M. J., \& van Ijzendoorn, M. H. (2007). Threat-related attentional bias in anxious and non-anxious individuals: A meta-analytic study. Psychological Bulletin, 133, 1-24.

Barlow, D. H. (1988). Anxiety and its disorders. New York: Guilford Press. 
Beck, A.T. \& Clark, D.A. (1997). An information processing model of anxiety: Automatic and strategic processes. Behaviour Research and Therapy, 35, 49-58.

Becker, E. S., Rinck, M., Magraf, J., \& Roth, W. T. (2001). The emotional Stroop effect in anxiety disorders — general emotionality or disorder specificity? Journal of Anxiety Disorders, 15, 147-159.

Becker, E. S., Roth, W. T., Andrich, M., \& Margraf, J. (1999). Explicit memory in anxiety disorders. Journal of Abnormal Psychology, 108, 153-163.

Behar, E., Alcaine, O., Zuellig, A. R., \& Borkovec, T. D. (2003). Screening for generalized anxiety disorder using the Penn State Worry Questionnaire: A receiver operating characteristic analysis. Journal of Behavior Therapy and Experimental Psychiatry, 34, 25-43.

Belzer, K. D., D’Zurilla, T. J., \& Maydeu-Olivares, A. (2002). Social problem solving and trait anxiety as predictors of worry in a college student population. Personality and Individual Differences, 33, 573-585.

Bernstein, I. H., \& Eveland, D. C. (1982). State vs trait anxiety: A case study in confirmatory factor analysis. Personality and Individual Differences, 3, 361-372.

Bieling, P. J., Antony, M. M., \& Swinson, R. P. (1998). The State- Trait Anxiety Inventory, Trait version: Structure and content re-examined. Behaviour Research and Therapy, 36, 777-788.

Blaxton, T. (1989). Investigating dissociations among memory measures: Support for a transfer-appropriate processing framework. Journal of Experimental Psychology: Learning, Memory, and Cognition, 15, 657-668. 
Blazer, D.G., Hughes, D., George, L. K., Swartz, M., \& Boyer, R. (1991). Generalized anxiety disorder. In L. N. Robins \& D. A. Regier (Eds.), Psychiatric disorders in America: The Epidemiologic Catchment Area Study (pp. 180-203). New York: Free Press.

Borkovec, T. D. (1985). Worry: A potentially valuable concept. Behaviour Research and Therapy, 23, 481-482.

Borkovec, T. D., Alcaine, O. M., \& Behar, E. (2004). Avoidance theory of worry and generalized anxiety disorder. In R. G. Heimberg, C. L. Turk, \& D. S. Mennin (Eds), Generalized anxiety disorder: Advances in research and practice (pp.77108). New York: Guilford Press.

Borkovec, T. D. \& Costello, E. (1993). Efficacy of applied relaxation and cognitivebehavioral therapy in the treatment of Generalized Anxiety Disorder. Journal of Consulting and Clinical Psychology, 61, 611-619.

Borkovec, T. D., Lyonfields, J. D., Wiser, S. L., \& Deihl, L. (1993). The role of worrisome thinking in the suppression of cardiovascular response to phobic imagery. Behaviour Research and Therapy, 31, 321-324.

Borkovec, T. D., \& Newman, M. G. (1999). Worry and Generalized Anxiety Disorder. In P. Salkovskis (Ed.), Comprehensive clinical psychology, Vol. 6, Adults : Clinical formulation and treatment (pp. 439-459). Oxford : Elsevier Science.

Borkovec, T. D., Newman, M. G., Pincus, A. L., \& Lytle R. (2002). A component analysis of cognitive-behavioural therapy for generalized anxiety disorder and the role of interpersonal problems. Journal of Consulting and Clinical Psychology, 70, 288-298. 
Borkovec, T. D., Ray, W. J., \& Stöber, J. (1998). Worry : A cognitive phenomenon intimately linked to affective, physiological, and interpersonal behavioral processes. Cognitive Therapy and Research, 22, 561-576.

Borkovec, T. D., Robinson, E., Pruzinsky, T., \& DePree, J. A. (1983). Preliminary exploration of worry: Some characteristics and processes. Behaviour Research and Therapy, 21, 9-16.

Borkovec, T. D., \& Roemer, L. (1995). Perceived functions of worry among Generalized Anxiety Disorder subjects: Distraction from more emotionally distressing topics? Journal of Behavioral Therapy and Experimental Psychiatry, 26, 25-30.

Bower, G. H. (2000). A brief history of memory research. In E. Tulving \& F. Craik (Eds.), The Oxford handbook of memory (pp. 3-32). New York, NY: Oxford University Press.

Bradley, B. P., Mogg, K., \& Millar, N. (2000). Covert and overt orienting of attention to emotional faces in anxiety. Cognition and Emotion, 14, 789-808.

Bradley, B. P., Mogg, K., Millar, N., \& White, J. (1995). Selective processing of negative information: Effects of clinical anxiety, concurrent depression, and awareness. Journal of Abnormal Psychology, 104, 532-536.

Bradley, B. P., Mogg, K.,White, J., Groom, C., \& de Bono, J. (1999). Attentional bias for emotional faces in generalised anxiety disorder. British Journal of Clinical Psychology, 38, 267-278.

Bradley, B. P., Mogg, K., \& Williams, R. (1995). Implicit and explicit memory for emotion-congruent information in clinical depression and anxiety. Behaviour Research and Therapy, 33, 755-770. 
Brown, T. A., Antony, M. M., \& Barlow, D. H. (1992). Psychometric properties of the Penn State Worry Questionnaire in a clinical anxiety disorders sample. Behaviour Research and Therapy, 30, 33-37.

Brown, T. A., Campbell, L. A., Lehman, Grisham, J. R., \& Mancill, R. B. (2001). Current and lifetime co-morbidity of DSM-IV anxiety and mood disorders in a large clinical sample. Journal of Abnormal Psychology, 110, 585-599.

Brown, T. A., Chorpita, L. A., \& Barlow, D. H. (1998). Structural relations among dimensions of the DSM-IV anxiety and mood disorders and dimensions of negative affect, positive affect, and autonomic arousal. Journal of Abnormal Psychology, 107, 179-192.

Buckley, T. C., Blanchard, E. B., \& Neill, W. T. (2000). Information processing and PTSD: A review of the empirical literature. Clinical Psychology Review, 28, 1041-1065.

Buhr, K., \& Dugas, M. J. (2002). The intolerance of uncertainty scale: Psychometric properties of the English version. Behaviour Research and Therapy, 40, 931-345.

Buhr, K., \& Dugas, M. J. (2006). Investigating the construct validity of intolerance of uncertainty and its unique relationship with worry. Journal of Anxiety Disorders, 20, 222-236.

Butler, G., Wells, A., \& Dewick, H. (1995). Differential effects of worry and imagery after exposure to a stressful stimulus: A pilot study. Behavioural and Cognitive Psychotherapy, 23, 45-56.

Carroll, J. B., Davies, P., \& Richman, B. (1971). Word frequency book. New York: American Heritage. 
Casey, L. M., Oei, T. P. S., \& Newcombe, P. A. (2004). An integrated cognitive model of panic disorder: The role of positive and negative cognitions. Clinical Psychology Review, 24, 529-555.

Cisler, J. M., Bacon, A. K., \& Williams, N. L. (2009). Phenomenological characteristics of attentional biases towards threat: A critical review. Cognitive Therapy and Research, 33, 221-234.

Cisler, J. M., \& Koster, E. H. W. (2010). Mechanisms of attentional biases towards threat in anxiety disorders: An integrative review. Clinical Psychology Review, 30, $203-$ 216.

Clark, D.A., \& Beck, A.T.(2010). Cognitive therapy of anxiety disorders: Science and practice. New York: Guilford Press.

Cohen, J. (1988). Statistical power analysis for the behavioral sciences (2nd edition). Hillsdale, NJ: Erlbaum.

Coles, M. E., Turk, C. L., \& Heimberg, R. G. (2007). Memory bias for threat in generalized anxiety disorder: The potential importance of stimulus relevance. Cognitive Behaviour Therapy, 36, 65-73.

Craik, F. I., \& Lockhart, R. S. (1972). Levels of processing: A framework for memory research. Journal of Verbal Learning and Verbal Behavior, 11, 671-684.

Craske, M. G., Rapee, R. M., Jackel, L., \& Barlow, D. H. (1989). Qualitative dimensions of worry in DSM-III-R generalized anxiety disorder subjects and nonanxious participants. Behaviour Research and Therapy, 27, 397-402.

Creamer, M., Foran, J., \& Bell, R. (1995). The Beck Anxiety Inventory in a nonclinical sample. Behaviour Research and Therapy, 33, 477-485. 
Crowne, D. P., \& Marlowe, D. (1960). A new scale of social desirability independent of psychopathology. Journal of Consulting Psychology, 24, 349-354.

Dalgleish, T. (1994). The relationship between anxiety and memory biases for material that has been selectively processed in a prior task. Behaviour Research and Therapy, 32, 227-231.

Davey, G. C. L. (1993). A comparison of three worry questionnaires. Behaviour Research and Therapy, 31, 51-56.

Davey, G. C. L. (1994a). Trait factors and ratings of controllability as predictors of worrying about significant life stressors. Personality and Individual Differences, 16, 379-384.

Davey, G. C. L. (1994b). Worrying, social problem-solving abilities, and social problemsolving confidence. Behaviour Research and Therapy, 32, 327-330.

Davey, G. C. L., Hampton, J., Farrell, J., \& Davidson, S. (1992). Some characteristics of worrying: Evidence for worrying and anxiety as separate constructs. Personality and Individual Differences, 13, 133-147.

Davey, G. C. L., Tallis, F., \& Capuzzo, N. (1996). Beliefs about the consequences of worrying. Cognitive Therapy and Research, 20, 499-520.

Deffenbacher, J. L. (1978). Worry, emotionality, and task-generated interference in test anxiety: An empirical test of attentional theory. Journal of Educational Psychology, 70, 248-254.

Derryberry, D., \& Reed, M. A. (2001). Attentional control, trait anxiety, and the regulation of irrelevant response information. Manuscript in review. 
Derryberry, D., \& Reed, M. A. (2002). Anxiety-related attentional biases and their regulation by attentional control. Journal of Abnormal Psychology, 111, 225-236.

Dugas, M. J., Brillon, P., Savard, P., Turcotte, J., Gaudet, A., Ladouceur, R., Leblanc, R., \& Gervais, N. J. (2010). A randomized clinical trial of cognitive-behavioral therapy and applied relaxation for adults with Generalized Anxiety Disorder. Behavior Therapy, 41, 46-58.

Dugas, M. J., Freeston, M. H., Blais, \& Ladouceur, R. (1993, November). Prise de décision et stimuli ambigus chez les personnes souffrant du trouble d'anxiété généralisée et de l'inquiétude excessive [Decision making and ambiguous stimuli in generalized anxiety disorder patients and high worriers]. Paper presented at the XVIth congress of the Société Québécoise de la Recherche en Psychologie, Québec, Canada.

Dugas, M. J., Freeston, M. H., Doucet, C. D., Lachance, S., \& Ladouceur, R. (1995). Structured versus free-recall measures: Effect on report of worry themes. Personality and Individual Differences, 18, 355-361.

Dugas, M. J., Freeston, M. H., \& Ladouceur, R. (1997). Intolerance of uncertainty and problem orientation in worry. Cognitive Therapy and Research, 21, 593-606.

Dugas, M. J., Freeston, M. H., Ladouceur, R, Rheaume, J., Gagnon, F., Thibodeau, N. (1998). Sociodemographic \& clinical features of GAD patients. Paper presented at the annual convention of the Association for the Advancement of Behavior Therapy, Washington, D.C.

Dugas, M. J., Freeston, M. H., Provencher, M. D., Lachance, S., Ladouceur, R., \& Gosselin, P. (2001). Le questionnaire sur l'inquiétude et l'anxiété: Validation 
dans des échantillons non cliniques et cliniques. [The Worry and Anxiety Questionnaire: Validation in clinical and nonclinical samples.] Journal de Thérapie Comportementale et Cognitive, 11, 31-36.

Dugas, M. J., Gagnon, F., Ladouceur, R., \& Freeston, H. (1998). Generalized anxiety disorder: A preliminary test of a conceptual model. Behaviour Research and Therapy, 36, 215-226.

Dugas, M. J., Hedayati, M., Karavidas, A., Buhr, K., Francis, K., \& Phillips, N. A. (2005). Intolerance of uncertainty and information processing: Evidence of biased recall and interpretations. Cognitive Therapy and Research, 29, 57-70.

Dugas, M. J., Ladouceur, R., Léger, E., Freeston, M. H., Langlois, F., Provencher, M., et al. (2003). Group cognitive-behavioral therapy for generalized anxiety disorder: Treatment outcome and long-term follow-up. Journal of Consulting and Clinical Psychology, 71, 821-825.

Dugas, M. J., Letarte, H., Rhéaume, J., Freeston, M. H., \& Ladouceur, R. (1995). Worry and problem solving: Evidence of a specific relationship. Cognitive Therapy and Research, 19, 109-120.

Dugas, M. J., Marchand, A., \& Ladouceur, R. (2005). Further validation of a cognitivebehavioral model of generalized anxiety disorder: Diagnostic and symptom specificity. Journal of Anxiety Disorders, 19, 329-343.

Dugas, M. J., \& Robichaud, M. (2007). Cognitive-behavioral treatment for generalized anxiety disorder: From science to practice. New York: Routledge. 
Dugas, M. J., Savard, P., Gaudet, A., Turcotte, J., Laugesen, N., Robichaud, M., Francis, K., \& Koerner, N. (2007). Can the components of a cognitive model predict the severity of Generalized Anxiety Disorder? Behavior Therapy, 38, 169-178.

Dugas, M. J., Schwartz, A., \& Francis, K. (2004). Intolerance of uncertainty, worry, and depression. Cognitive Therapy and Research, 28, 835-842.

Dupuy, J.-B., Beaudoin, S., Rhéaume, J., Ladouceur, R., \& Dugas, M. J. (2001). Worry: daily self-report in clinical and non-clinical populations. Behaviour Research and Therapy, 39, 1249-1255.

D’Zurilla, T. J., \& Nezu, A. M. (1999). Problem-solving therapy: A social competence approach to clinical intervention ( $2^{\text {nd }}$ ed. ). New York: Springer.

Eysenck, M. W. (1992). Anxiety: The cognitive perspective. Hillsdale, NJ: Lawrence Erlbaum Associates.

Eysenck, M. W., \& Byrne, A. B. (1994). Implicit memory bias, explicit memory bias, and anxiety. Cognition and Emotion, 8, 415-431.

Eysenck, M. W., Mogg, K., May, J., Richards, A., \& Mathews, A. (1991). Bias in interpretation of ambiguous sentences related to threat in anxiety. Journal of Abnormal Psychology, 100, 144-150.

Eysenck, M. W., Payne, S., \& Derakshan, N. (2007). Anxiety and cognitive performance: Attentional control theory. Emotion, 7, 336-353.

Field, A. (2005) Discovering statistics using SPSS (2 ${ }^{\text {nd }}$ ed.). London: Sage Publications.

Finucane, M.L., Alhakami, A., Slovic, P., \& Johnson, S.M. (2000). The affect heuristic in judgments of risks and benefits. Journal of Behavioural Decision Making, 13, 117. 
Foa, E. B., \& Kozak, M. J. (1986). Emotional processing of fear: Exposure to corrective information. Psychological Bulletin, 99, 20-35.

Francis, W. N., \& Kucera, H. (1982). Frequency analysis of English usage. Boston: Houghton Mifflin.

Freeston, M.H., Rhéaume, J., Letarte, H., Dugas, M.J., \& Ladouceur, R. (1994). Why do people worry? Personality and Individual Differences, 17, 791-802.

Friedman, B. H., Thayer, J. F., \& Borkovec, T. D. (2000). Explicit memory bias for threat words in generalized anxiety disorder. Behavior Therapy, 31, 745-756.

Frost, R. O., Marten, P., Lahart, C., \& Rosenblate, R. (1990). The dimensions of perfectionism. Cognitive Therapy and Research, 14, 449-468.

Gilhooly, K. J., \& Logie, R. H. (1980). Meaning-dependent ratings of imagery, age of acquisition, familiarity, and concreteness for 387 ambiguous words. Behavior Research Methods and Instrumentation, 12, 428-450.

Golombok, S., Stavrou, A., Bonn, J., Mogg, K., Critchlow, S., \& Rust, J. (1991). Effects of diazepam on anxiety-related cognition. Cognitive Research and Therapy, 15, $459-467$.

Gosselin, P., Langlois, F., Freeston, M. H., Ladouceur, R., Dugas, M. J., \& Pelletier, O. (2002). Le Questionnaire d'Évitement Cognitif (QEC): Développement et validation auprès d'adultes et d'adolescents. [The Cognitive Avoidance Questionnaire (CAQ): Development and validation among adult and adolescent samples]. Journal de Thérapie Comportementale et Cognitive, 12, 24-37. 
Graf, P., \& Mandler, G. (1984). Activation makes words more accessible, but not necessarily more retrievable. Journal of Verbal Learning and Verbal Behavior, $23,553-568$.

Graf, P., \& Ryan, L. (1990). Transfer-appropriate processing for implicit and explicit memory. Journal of Experimental Psychology: Learning, Memory, and Cognition, 16, 978-992.

Green, M. W. \& McKenna, F. P. (1996). Making semantic judgements about affectively valanced words: A new test of processing bias in anxiety. Personality and Individual Differences, 20, 429-436.

Harrison, L. K. \& Turpin, G. (2003). Implicit memory bias and trait anxiety: A psychophysiological analysis. Biological Psychology, 62, 97-114.

Hautus, M. (1995). Corrections for extreme proportions and their biasing effects on estimated values of d'. Behavior Research Methods, Instruments, \& Computers, $27,46-51$.

Hayes, S., Hirsch, C. R., Krebs, G., \& Mathews, A. (2010). The effects of modifying interpretation bias on worry in generalized anxiety disorder. Behaviour Research and Therapy, 48, 171-178.

Heinecke, N., Koerner, N., Dugas, M. J., \& Mogg, K. (2006, July). The relation between intolerance of uncertainty and attentional biases. Poster presented at the annual meeting of the British Association for Behavioural and Cognitive Psychotherapies, Warwick, U. K.

Hirsch, C. R., \& Clark, D. M. (2004). Information-processing bias in social phobia. Clinical Psychology Review, 24, 799-825. 
Hirsch, C. R., Hayes, S., \& Mathews, A. (2009). Looking on the bright side: Accessing benign meanings reduces worry. Journal of Abnormal Psychology, 118, 44-54.

Holaway, R. M., Heimberg, R. G., \& Coles, M. E. (2006). A comparison of intolerance of uncertainty in analogue obsessive-compulsive disorder and generalized anxiety disorder. Journal of Anxiety Disorders, 20, 158-174.

Hoyer, J., Becker, E. S., \& Roth, W. T. (2001). Characteristics of worry in GAD patients, social phobics, and controls. Depression and Anxiety, 13, 89-96.

Jacobson, N. S., \& Truax, P. (1991). Clinical significance: a statistical approach to defining meaningful change in psychotherapy research. Journal of Consulting and Clinical Psychology, 59, 12-19.

Jacoby, L. L. (1983). Remembering the data: Analyzing interactive processes in reading. Journal of Verbal Learning and Verbal Behavior, 22, 485-508.

Jacoby, L. L., \& Dallas, M. (1981). On the relationship between autobiographical memory and perceptual learning. Journal of Experimental Psychology: General, 110, 306-340.

Kaspi, S. P., McNally, R., J., \& Amir, N. (1995). Cognitive processing of emotional information in post-traumatic stress disorder. Cognitive Therapy and Research, 19, 433-444.

Kessler, R.C., McGonagle, K. A., Zhao, S., Nelson, C. B., Hughes, M., Eshleman, S., et al. (1994). Lifetime and 12 month prevalence of DSM-III-R psychiatric disorders in the United States: Results from the National Comorbidity Study. Archives of General Psychiatry, 51, 8-19. 
Kircanski, K., Craske, M. G., \& Bjork, R. A. (2008). Thought suppression enhances memory bias for threat material. Behaviour Research and Therapy, 46, 462-476.

Klein, S. B., \& Loftus, J. (1988). The nature of self-referent encoding: The contributions of elaborative and organizational processes. Journal of Personality and Social Psychology, 55, 5-11.

Koerner, N., \& Dugas, M. J. (2007). Intolerance of uncertainty, cognitive avoidance and their relation to interpretive processing. Manuscript submitted for publication.

Koerner, N., \& Dugas, M. J. (2008). An investigation of appraisals in individuals vulnerable to excessive worry: The role of intolerance of uncertainty. Cognitive Therapy and Research, 32, 619-638.

Koerner, N., Dugas, M. H., Savard, P., Gaudet, A., Turcotte, J., \& Marchand, A. (2004). The economic burden of anxiety disorders in Canada. Canadian Psychology, 45, $191-201$

Ladouceur, R., Dugas, M. J. \& Freeston, M. H., Léger, E., Gagnon, F., \& Thibodeau, N. (2000). Efficacy of a cognitive-behavioral treatment for generalized anxiety disorder: Evaluation in a controlled clinical trial. Journal of Consulting and Clinical Psychology, 68, 957-964.

Ladouceur, R., Dugas, M. J., Freeston, M. H., Rhéaume, J., Blais, J.-M., Boisvert, J.-M., Gagnon, F., et al. (1999). Specificity of generalized anxiety disorder symptoms and processes. Behavior Therapy, 30, 191-207.

Ladouceur, R., Gosselin, P., \& Dugas, M. J. (2000). Experimental manipulation of intolerance of uncertainty: A study of a theoretical model of worry. Behaviour Research and Therapy, 38, 933-941. 
Lang, P.J., Bradley, M.M., \& Cuthbert, B.N. (2001). International Affective Picture System (IAPS): Instruction manual and affective ratings. Technical Report A-5, The Center for Research in Psychophysiology, University of Florida.

Lecrubier, Y., Sheehan, D. V., Weiller, E., Amorim, P., Bonora, I., Harnett Sheehan, K., Janavs, J., \& Dunbar, G. C. (1997). The Mini-International Neuropsychiatric Interview (MINI). A short diagnostic structured interview: reliability and validity according to the CIDI.E.uropean Psychiatry, 12, 224-231.

Light, L. L., \& Singh, A. (1987). Implicit and explicit memory in young and older adults. Journal of Experimental Psychology: Learning, Memory, and Cognition, 13, 531541.

Lundh, L., \& Öst, L. (1996). Recognition bias for critical faces in social phobics. Behaviour Research and Therapy, 34, 787-794.

MacLeod, A. K., Williams, J. M. G., \& Bekerian, D. A. (1991). Worry is reasonable: The role of explanations in pessimism about future personal events. Journal of Abnormal Psychology, 100, 478-486.

MacLeod, C., \& Mathews, A. (1988). Anxiety and the allocation of attention to threat. Quarterly Journal of Experimental Psychology, 40, 653-670.

MacLeod, C., Matthews, A., \& Tata, P. (1986). Attentional bias in emotional disorders. Journal of Abnormal Psychology, 95, 15-20.

MacLeod, C., \& McLaughlin, K. (1995). Implicit and explicit memory bias in anxiety: A conceptual replication. Behaviour Research and Therapy, 33, 1-14.

MacLeod, C. \& Rutherford, E. M. (1992). Anxiety and the selective processing of emotional information: Mediating roles of awareness, trait and state variables, 
and personal relevance of stimulus materials. Behaviour Research and Therapy, 30, 479-491.

MacLeod, C. \& Rutherford, E. (2004). Information-processing approaches: Assessing the selective functioning of attention, interpretation, and retrieval. In R. G. Heimberg, C. L. Turk \& D. S. Mennin (Eds), Generalized anxiety disorder: Advances in research and practice (pp.109-142). New York: Guilford Press.

Maier, W., Gansicke, M., Freyberger, H. J., Linz, M., Heun, R., \& Lecrubier, Y. (2000). Generalized anxiety disorder (ICD-10) in primary care from a cross-cultural perspective: A valid diagnostic entity? Acta Psychiatrica Scandinavica, 101, 2936.

Manguno-Mire, G. M., Constans, J. I., \& Geer, J. H. (2005). Anxiety-related differences in affective categorizations of lexical stimuli. Behaviour Research and Therapy, 43, 197-213.

Martin, M., Ward, J. C., \& Clark, D. M. (1983). Neuroticism and the recall of positive and negative personality information. Behaviour Research and Therapy, 5, 495503.

Martin, M., Williams, R., \& Clark, D. (1991). Does anxiety lead to selective processing of threat-related information? Behaviour Research and Therapy, 29, 147-160.

Mathews, A., \& Klug, F. (1993). Emotionality and interference with colour-naming in anxiety. Behaviour Research and Therapy, 31, 57-62.

Mathews, A., \& Mackintosh, B. (1998). A cognitive model of selective processing in anxiety. Cognitive Therapy and Research, 22, 539-560. 
Mathews, A., \& MacLeod, C. (1985). Selective processing of threat cues in anxiety states. Behaviour Research and Therapy, 23, 563-569.

Mathews, A., \& MacLeod, C. (1986). Discrimination of threat cues without awareness in anxiety states. Journal of Abnormal Psychology, 95, 131-138.

Mathews, A., \& MacLeod, C. (2002). Induced processing biases have causal effects on anxiety. Cognition and emotion, 16, 331-354.

Mathews, A., \& MacLeod, C. (2005). Cognitive vulnerability to emotional disorders. Annual Review of Clinical Psychology, 1, 167-195.

Mathews, A., May, J., Mogg, K., \& Eysenck, M. (1990). Attentional bias in anxiety: Selective search or defective filtering? Journal of Abnormal Psychology, 99, 166173.

Mathews, A., Mogg, K., Kentish, J., \& Eysenck, M. (1995). Effect of psychological treatment on cognitive bias in generalized anxiety disorder. Behaviour Research and Therapy, 33, 293-303.

Mathews, A., Mogg, K., May, J., \& Eysenck, M. (1989). Implicit and explicit memory bias in anxiety. Journal of Abnormal Psychology, 98, 236-240.

Mathews, A., Richards, A., \& Eysenck, M. (1989). Interpretation of homophones related to threat in anxiety states. Journal of Abnormal Psychology, 98, 31-34.

Mathews, A., Ridgeway, V., Cook, E., \& Yiend, J. (2007). Inducing a benign interpretational bias reduces trait anxiety. Journal of Behavior Therapy and Experimental Psychiatry, 38, 225-236.

Mathews, A., Ridgeway, V., \& Williamson, D. A. (1996). Evidence for attention to threatening stimuli in depression. Behaviour Research and Therapy, 34, 695-705. 
McNally, R. J. (1994). Cognitive bias in panic disorder. Current Directions in Psychological Science, 3, 129-132.

McNally, R. J. (1995). Automaticity and the anxiety disorders. Behaviour Research and Therapy, 33, 747-754.

Medical Research Council (MRC) Psycholinguistic Database, http://www.psy.uwa.edu.au/mrcdatabase/uwa_mrc.htm

Metzger, R. L., Miller, M. L., Cohen, M., Sofka, M., \& Borkovec, T. D. (1990). Worry changes decision making: The effect of negative thoughts on cognitive processing. Journal of Clinical Psychology, 46, 78-88.

Meyer, T. J., Miller, M. L., Metzger, R. L., \& Borkovec, T. D. (1990). Development and Validation of the Penn State Worry Questionnaire. Behaviour Research and Therapy, 28, 487-495.

Molina, S., \& Borkovec, T. D. (1994). The Penn State Worry Questionnaire: Psychometric properties and associated characteristics. In: G. C. L.. Davey \& F. Tallis (Eds.), Worrying: Perspectives on theory, assessment and treatment. New York: John Wiley \& Sons.

Mogg, K., \& Bradley, B. P. (2005). Attentional bias in Generalized Anxiety Disorder versus Depressive Disorder. Cognitive Therapy and Research, 29, 29-45.

Mogg, K., \& Bradley, B. P., \& Hallowell, N. (1994). Attentional bias to threat: Roles of trait anxiety, stressful events, and awareness. Quarterly Journal of Experimental Psychology: Human Experimental Psychology, 47, 841-864. 
Mogg, K., Bradley, B. P., \& Williams, R. (1995). Attentional bias in anxiety and depression: the role of awareness. British Journal of Clinical Psychology, 34, $17-$ 36.

Mogg, K., Bradley, B. P., Williams, R., \& Mathews, A. (1993). Subliminal processing of emotional information in anxiety and depression. Journal of Abnormal Psychology, 102, 304-311.

Mogg, K., \& Mathews, A. (1990). Is there a self-referent mood-congruent recall bias in anxiety? Behaviour Research and Therapy, 28, 91-92.

Mogg, K., Mathews, A., \& Eysenck, M. (1992). Attentional bias in clinical anxiety states. Cognition and Emotion, 6, 149-159.

Mogg, K., Mathews, A., \& Weinman, J. (1987). Memory bias in clinical anxiety. Journal of Abnormal Psychology, 96, 94-98.

Mogg, K., Mathews, A., \& Weinman, J. (1989). Selective processing of threat cues in anxiety states: A replication. Behaviour Research and Therapy, 27, 317-323.

Mogg, K., Millar, N., \& Bradley, B. P. (2000). Biases in eye movements to threatening facial expressions in generalised anxiety disorder and depressive disorder. Journal of Abnormal Psychology, 19, 695-704.

Morris, C. D., Bransford, J. D., \& Franks, J. J. (1977). Levels of processing versus transfer-appropriate processing. Journal of Verbal Learning and Verbal Behavior, 16, 519-533.

Morris, L. W., \& Liebert, R. M. (1970). Relationship of cognitive and emotional components of test anxiety to physiological arousal and academic performance. Journal of Consulting and Clinical Psychology, 35, 332-337. 
Muller, J., \& Roberts, J. E. (2005). Memory and attention in Obsessive-Compulsive Disorder: A review. Journal of Anxiety Disorders, 19, 1-28.

Norton, P. J. (2005). A psychometric analysis of the intolerance of uncertainty scale among four racial groups. Journal of Anxiety Disorders, 19, 699-707.

Nugent, K., \& Mineka, S. (1994). The effect of high and low trait anxiety on implicit and explicit memory tasks. Cognition and Emotion, 8, 147-163.

Olatunji, B. O., Broman-Fulks, J. J., Bergman, S. M., Green, B. A., \& Zlomke, K. R. (2010). A taxometric investigation of the latent structure of worry:

Dimensionality and associations with depression, anxiety, and stress. Behavior Therapy, 41, 212-228.

Oldenburg, C., Lundh, L-G., \& Kivisto, P. (2002). Explicit and implicit memory, trait anxiety, and repressive coping style. Personality and Individual Differences, 32, 107-119.

Otto, M. W., McNally, R. J., Pollack, M. H., Chen, E., \& Rosenbaum, J. F. (1994). Hemispheric laterality and memory bias for threat in anxiety disorders. Journal of Abnormal Psychology, 103, 828-831.

Paivio, A., Yuille, J. C., \& Madigan, S. A. (1968). Concreteness, imagery, and meaningfulness values for 925 nouns. Journal of Experimental Psychology Monograph Supplement, 76, No. 1, 1-10.

Perruchet, P., \& Baveux, P. (1989). Correlational analyses of explicit and implicit memory performance - word stems are influenced by explicit memory. Memory \& Cognition, 17, 77-86. 
Pury, L. S., \& Mineka, S. (2001). Differential encoding of affective and nonaffective content information in trait anxiety. Cognition and Emotion, 15, 659-693.

Radloff, L. S. (1977). The CES-D Scale: A self-report depression scale for research in the general population. Applied Psychological Measurement, 1, 385-401.

Radomsky, A. S., \& Rachman, S. (1999). Memory bias in obsessive-compulsive disorder (OCD). Behaviour Research and Therapy, 37, 605-618.

Rapee, R. M. (1991). Generalized anxiety disorder: A review of clinical features and theoretical concepts. Clinical Psychology Review, 11, 419-440.

Rassin, E., Merckelbach, H., \& Muris, P. (2000). Paradoxical and less paradoxical effects of thought suppression: A critical review. Clinical Psychology Review, 20, 973995.

Raven, J. C., Court, J. H., \& Raven, J. (1977). Manual for Raven's progressive matrices and vocabulary scales. London: Lewis.

Reidy, J. (2004). Trait anxiety, trait depression, worry, and memory. Behaviour Research and Therapy, 42, 937-948.

Reidy, J. \& Richards, A. (1997a). Anxiety and memory: A recall bias for threatening words in high anxiety. Behaviour Research and Therapy, 35, 531-542.

Reidy, J. \& Richards, A. (1997b). A memory bias for threat in high-trait anxiety. Personality and Individual Differences, 23, 653-663.

Reynolds, W. M. (1982). Development of reliable and valid short forms of the MarloweCrowne Social Desirability Scale. Journal of Clinical Psychology, 38, 119-125. 
Richards, A. \& French, C. C. (1991). Effects of encoding and anxiety on implicit and explicit memory performance. Personality and Individual Differences, 12, 131139.

Richards, A., French, C. C., Adams, C., Eldridge, M., \& Papadopolou, E. (1999). Implicit memory and anxiety: Perceptual identification of emotional stimuli.e.uropean Journal of Cognitive Psychology, 11, 67-86.

Rinck, M., Becker, E. S., Kellerman, J., \& Roth, W. T. (2003). Selective attention in anxiety: Distraction and enhancement in visual search. Depression and Anxiety, $18,18-28$.

Riskind, J. H., Moore, R., Harman, B., Hohmann, A. A., Beck, A. T., \& Stewart, B. (1991). The relation of Generalized Anxiety Disorder to depression in general and dysthymic disorder in particular. In R. M. Rapee \& D. H. Barlow (Eds.), Chronic anxiety: Generalized anxiety disorder and mixed anxiety-depression (pp. 153171). New York: Guilford.

Roediger, H. L. (1990). Implicit memory: Retention without remembering. American Psychologist, 45, 1043-1056.

Roediger, H. L., \& Amir, N. (2005). Implicit memory tasks: Retention without conscious recollection. In A. Wenzel \& D. C. Rubin (Eds.), Cognitive methods and their application to clinical research (pp. 121-127). Washington, DC: United Book Press.

Roediger, H. L., \& McDermott, K. B. (1993). Implicit memory in normal human subjects. In F. Boller \& J. Grafman (Eds.), Handbook of Neuropsychology. New York: Elsevier Science. 
Roemer, L., Molina, S., \& Borkovec, T. D. (1997). An investigation of worry content among generally anxious individuals. The Journal of Nervous and Mental Disease, 185, 314-319.

Roget's College Thesaurus, Revised Edition (2000). USA: Random House.

Ruscio, A. M. (2002). Delimiting the boundaries of generalized anxiety disorder: Differentiating high worriers with and without GAD. Journal of Anxiety Disorders, 16, 377-400.

Ruscio, A. M., \& Borkovec, T. D. (2004). Experience and appraisal of worry among high worriers with and without generalized anxiety disorder. Behaviour Research and Therapy, 42, 1469-1482.

Ruscio, A. M., Borkovec, T. D, \& Ruscio, J. (2001). A taxometric study of the latent structure of worry. Journal of Abnormal Psychology, 110, 413-422.

Russo, R., Fox, E., \& Bowles, R. J. (1999). On the status of implicit memory bias in anxiety. Cognition and Emotion, 13, 435-456.

Schacter, D. L. (1987). Implicit memory: History and current status. Journal of Experimental Psychology: Learning, Memory, and Cognition, 13, 501-518.

Schacter, D. L., Chiu, C.-Y. P., \& Ochsner, K. N. (1993). Implicit memory: A selective review. Annual Review of Neuroscience, 16, 159-182.

Schneider, W., Eschman, A., \& Zuccolotto, A. (2002). E-Prime user's guide. Pittsburgh: Psychology Software Tools Inc.

Schwartz, G. E., Davidson, R. J., \& Goleman., D. J. (1978). Patterning of cognitive and somatic processes in the self-regulation of anxiety: Effects of meditation versus exercise. Psychosomatic medicine, 40, 321-328. 
Seipp, B. (1991). Anxiety and academic performance: A meta-analysis of findings. Anxiety Research, 4, 27-41.

Sexton, K., \& Dugas, M. J. (2007, November). Cross-cultural validity of the Intolerance of Uncertainty Scale. Poster presented at the annual meeting of the British Association for Behavioural and Cognitive Psychotherapies, Philadelphia, P. A.

Sexton, K. A., \& Dugas, M. J. (2009a). An investigation of factors associated with cognitive avoidance in worry. Cognitive Therapy and Research, 33, 150-162.

Sexton, K. A., \& Dugas, M. J. (2009b). Defining distinct negative beliefs about uncertainty: Validating the factor structure of the Intolerance of Uncertainty Scale. Psychological Assessment, 21, 176-186.

Sexton, K. A., \& Dugas, M. J. (2008). The Cognitive Avoidance Questionnaire: Validation of the English translation. Journal of Anxiety Disorders, 22, 355-370.

Shadick, R. N., Roemer, L., Hopkins, M. B., \& Borkovec, T. D. (1991). A description of patients diagnosed with DSM-III-R generalized anxiety disorder. The Journal of Nervous and Mental Disease, 178, 588-591.

Sheehan, D. V., Lecrubier, Y., Harnett Sheehan, K., Amorim, P., Janavs, J., Weiller, E., Hergueta, T., Baker, R., Dunbar, G. C. (1998). The Mini-International Neuropsychiatric Interview (M.I.N.I.): The development and validation of a structured diagnostic psychiatric interview for DSM-IV and ICD-10. Journal of Clinical Psychiatry, 59, Suppl 20, 22-33.

Sheehan, D. V., Lecrubier, Y., Harnett Sheehan, K., Janavs, J., Weiller, E., Keskiner, A., Schinka, J., Knapp, E., Sheehan, M. F., \& Dunbar, G. C. (1997). The validity of 
the Mini-International Neuropsychiatric Interview (MINI) According to the SCID-P and its reliability. European Psychiatry, 12, 232-241.

Smith, E. R., \& Branscombe, N. R. (1988). Category accessibility as implicit memory. Journal of Experimental Social Psychology, 24, 490-504.

Spielberger, C. D. (1985). Anxiety, cognition, and affect: A state-trait perspective. In A. H. Tuma \& J. D. Maser (Eds.), Anxiety and the anxiety disorders (pp. 171-182). Hillsdale, NJ: Erlbaum.

Spielberger, C.D., Gorsuch, R.L., Lushene, R., Vagg, P.R., \& Jacobs, G.A. (1977). StateTrait Inventory for adults: Self-evaluation questionnaire STAI Form Y-1 and Form Y-2. Redwood City, CA: Mind Garden.

Spreen, O., \& Schulz, R. W. (1966). Parameters of abstraction, meaningfulness, and pronounciability for 329 nouns. Journal of Verbal Learning and Verbal Behavior, 5, 459-468.

Stanislaw, H., \& Todorov, N. (1999). Calculation of signal detection theory measures. Behavior Research Methods, Instruments, \& Computers, 31, 137-149.

Starcevic, V., Berle, D., Milicevic, D., Hannan, A., Lamplugh, C., \& Eslick, G. D. (2007). Pathological worry, anxiety disorders and the impact of co-occurrence with depressive and other anxiety disorders. Journal of Anxiety Disorders, 21, 1016-1027.

Steptoe, A., \& Kearsley, N. (1990). Cognitive and somatic anxiety. Behaviour Research and Therapy, 28, 75-81. 
Stöber, J., \& Joormann, J. (2001). Worry, procrastination, and perfectionism:

Differentiating amount of worry, pathological worry, anxiety, and depression. Cognitive Therapy and Research, 25, 49-60.

Stokes, C., \& Hirsch, C. R. (2010). Engaging in imagery versus verbal processing of worry: Impact on negative intrusions in high worriers. Behaviour Research and Therapy, 48, 418-423.

Symons, C. S., \& Johnson, B. T. (1997). The self-reference effect in memory: A metaanalysis. Psychological Bulletin, 121, 371-394.

Tabachnik, B. G., \& Fidell, L. S. (2001). Using multivariate statistics (4 ${ }^{\text {th }}$ ed.). Boston: Allyn \& Bacon.

Taghavi, M. R., Neshat-Doost, H. T., Moradi, A. R., Yule, W., \& Dalgleish, T. (1999). Biases in visual attention in children and adolescents with clinical anxiety and mixed anxiety-depression. Journal of Abnormal Child Psychology, 27, 215-223.

Tallis, F., Davey, G. C. L., \& Capuzzo, N. (1994). The phenomenology of nonpathological worry: A preliminary investigation. In G. C. L. Davey, \& F. Tallis (Eds.), Worrying: Perspectives on theory, assessment and treatment (pp. 61-89). New York: Wiley.

Tallis, F., Eysenck, M., \& Mathews, A. (1991). Elevated evidence requirements and worry. Personality and Individual Differences, 12, 21-27.

Tarsia, M., Power, M. J., \& Sanavio, E. (2003). Implicit and explicit memory biases in mixed anxiety-depression. Journal of Affective Disorders, 77, 213-225.

Thorndike, E., \& Lorge, I. (1944). The teacher's handbook of 30,000 words. New York: Columbia University Teachers' College Press. 
U.S. Department of Health and Human Services (1980). International Classification of Diseases, $9^{\text {th }}$ Revision: Clinical modification $\left(2^{\text {nd }}\right.$ ed.) Washington, DC: US Government Printing Office.

Vrana, S. R., Cuthbert, B. N., \& Lang, P. J. (1986). Fear imagery and text processing. Psychophysiology, 23, 247-253.

Vrana, S. R., Roodman, A., \& Beckham, J. C. (1995). Selective processing of traumarelevant words in post-traumatic stress disorder. Journal of Anxiety Disorders, 9, $515-530$.

Weissman, A. N. (1980). Assessing depressogenic attitudes: A validation study. Paper presented at the $51^{\text {st }}$ Annual Meeting of the Eastern Psychological Association, Hartford, CT.

Wells, A., \& Carter, K. (1999). Preliminary tests of a cognitive model of generalized anxiety disorder. Behaviour Research and Therapy, 37, 585-594.

Wells, A., \& Papageorgiou, C. (1995). Worry and the incubation of intrusive images following stress. Behaviour Research and Therapy, 33, 579-583.

Wegner, D. M. (1994). Ironic processes of mental control. Psychological Review, 101, $34-52$.

Wegner, D. M., Schneider, D. J., Carter, S. R., \& White, T. L. (1987). Paradoxical effects of thought suppression. Journal of Personality and Social Psychology, 53, 5-13.

Wittchen, H. U. (2002). Generalized anxiety disorder: Prevalence, burden, and cost to society. Depression and Anxiety, 16, 162-171.

Williams, J. M. G., Mathews, A., \& MacLeod, C. (1996). The emotional Stroop task and psychopathology. Psychological Bulletin, 120, 3-24. 
Williams, J. M. G., Watts, F. N., MacLeod, C., \& Mathews, A., (1997). Cognitive psychology and emotional disorders, 2nd edition. Wiley: Chichester.

Zebb, B. J., \& Beck, J. G. (1998). Worry versus anxiety: Is there really a difference? Behavior Modification, 22, 45-61. 
Appendix A

Study 1: Set 1 Pilot Words Used in the Concreteness, Familiarity, Imageability, Uncertainty, and Valence Questionnaires

\begin{tabular}{|c|c|c|c|}
\hline $\mathbf{1}$ & $\mathbf{2}$ & $\mathbf{3}$ & $\mathbf{4}$ \\
\hline unforeseen & unsafe & interval & disaster \\
\hline platform & sketch & inspiration & underaged \\
\hline despised & chance & combination & mortified \\
\hline inhabit & answer & theoretical & unload \\
\hline integrity & epidemic & casualty & inferior \\
\hline unsanitary & joyful & uncertain & unlisted \\
\hline additional & alley & accepted & feverish \\
\hline symmetrical & possibility & mutilated & actual \\
\hline unsure & nausea & intelligence & unselfish \\
\hline emergency & generous & unloved & lethal \\
\hline literal & unafraid & injury & interior \\
\hline rude & substitute & destroy & many \\
\hline surprising & methodical & search & pleasant \\
\hline compliment & collision & install & caring \\
\hline lonely & undecided & rural & unpeel \\
\hline ignorant & measure & information & great \\
\hline unanticipated & undo & massacre & unlock \\
\hline infection & early & imprecise & unsettled \\
\hline perhaps & universal & unhealthy & unique \\
\hline vague & sandy & dangerous & attractive \\
\hline unpin & understanding & unclear & incompetent \\
\hline import & pitiful & inadequate & under \\
\hline heroic & maimed & probability & ingredient \\
\hline ambiguous & immature & idiot & annual \\
\hline guess & virus & untalented & isolated \\
\hline questionable & happy & inanimate & ridiculed \\
\hline intern & neutral & adored & every \\
\hline origin & unspecified & unsweetened & admired \\
\hline popular & ineffective & hated & assault \\
\hline unexpected & inconclusive & pathetic & because \\
\hline past & disapproval & uneducated & \\
\hline
\end{tabular}

Note. Words are in the randomized order used in the pilot testing questionnaires listed above; this order reads from top to bottom, beginning with column 1 . Set 1 includes 20 positive, 20 social threat, 20 physical threat, 20 uncertain, and 43 neutral words, for a total of 123 words. 
Appendix B

Study 1: Set 2 Pilot Words Used in the Concreteness, Familiarity, Imageability, Uncertainty, and Valence Questionnaires

\begin{tabular}{|c|c|c|c|}
\hline $\mathbf{1}$ & $\mathbf{2}$ & $\mathbf{3}$ & $\mathbf{4}$ \\
\hline unwell & criticized & unresolved & convulsion \\
\hline customary & positive & vertical & unpredictable \\
\hline comprehensive & illness & unknown & inventory \\
\hline puzzling & unearth & horizontal & changeable \\
\hline loser & hesitation & death & contaminated \\
\hline underwater & unplanned & pain & faithful \\
\hline applause & toxic & selection & insensitive \\
\hline charming & random & unrecorded & brutality \\
\hline poisonous & stupid & insecure & foolish \\
\hline unflavoured & quantity & beautiful & sickness \\
\hline mangled & failure & approval & friendly \\
\hline unashamed & similar & fluctuating & debatable \\
\hline worthy & unkind & instruction & unattractive \\
\hline unroll & irregular & humiliated & mathematical \\
\hline indefinite & violence & disease & ignored \\
\hline introverted & supply & peaceful & fatal \\
\hline unit & torture & inept & confident \\
\hline mysterious & unpack & indistinct & doubtful \\
\hline geographical & entire & praise & blink \\
\hline tentative & good & apparent & about \\
\hline inside & kill & fracture & alphabet \\
\hline unfold & unglued & delightful & inexact \\
\hline untreatable & love & unpaved & embarrassed \\
\hline enthusiastic & tricky & disgrace & outside \\
\hline modern & unilingual & untie & injection \\
\hline offended & indicate & reciprocal & exterior \\
\hline unstick & square & optimistic & recent \\
\hline likeable & verse & unsold & mocked \\
\hline varying & coward & unsophisticated & talented \\
\hline accident & presence & wonderful & height \\
\hline ashamed & maybe & nation & \\
\hline
\end{tabular}

Note. Words are in the randomized order used in the pilot testing questionnaires listed above; this order reads from top to bottom, beginning with column 1 . Set 2 includes 20 positive, 20 social threat, 20 physical threat, 20 uncertain, and 43 neutral words, for a total of 123 words. 
Appendix C

Study 1 Concreteness Questionnaire

\section{CONC}

Words differ in the extent to which they refer to concrete objects, persons, places or things that can be seen, heard, felt, smelled, or tasted, as contrasted with abstract concepts that cannot be experienced by our senses. In this questionnaire you will be asked to rate a list of words with respect to their "concreteness" in terms of sensory experience.

Any word that refers to objects, materials, or persons, should be given a high concreteness rating (at the upper end of the numerical scale). Any word that refers to an abstract concept that cannot be experienced by the senses should be given a low concreteness rating (at the lower end of the numerical scale).

For example, think of the word "Carpet", which can be experienced by our senses and therefore should be rated as high concrete; the word "Option" cannot be experienced by the senses and therefore should be rated as low concrete (or abstract). Words can make you think of other words by association, but it is important that you only rate the concreteness of each specific word.

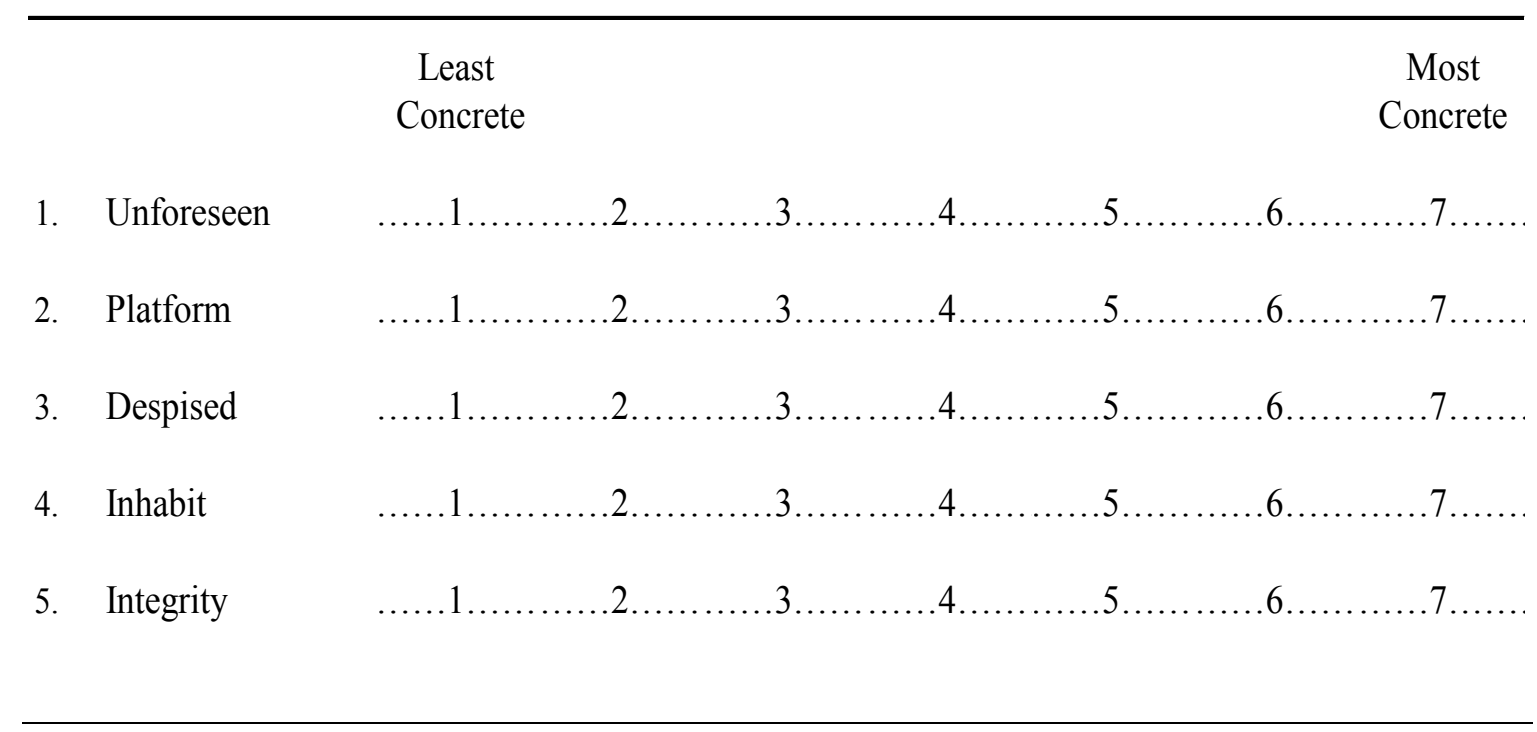

Note. In order to conserve space and limit the length of the Appendices, only a sample of the first page of this questionnaire is provided. The remaining pages are identically formatted (with the exception of the instructions), and include the remaining study stimuli, in the order presented in Appendices A and B. 
Appendix D

Study 1 Familiarity Questionnaire

\section{FAM}

This is a questionnaire to find out how often you have come in contact with certain words. Below is a list of words. Please rate each one on the number of times you have experienced it by circling a number under one of the five adjectives provided. The five possible adjectives are NEVER, RARELY, SOMETIN OFTEN, and VERY OFTEN. This means you have seen or heard or used the particular word in writing or in speech either:

NEVER................. You have never seen or heard or used the word in your life

RARELY.............. You have seen or heard or used the word at least once before, but only rarely

SOMETIMES...... You have sometimes seen or heard or used the word, but not often

OFTEN.................. You have often seen or heard or used the word, but not very often

VERY OFTEN..... You have seen or heard or used the word nearly every day of your life

Do not be concerned if you are unable to give a definition of some of the words.

Simply rate each one regardless of its meaning.

\begin{tabular}{|c|c|c|c|c|c|c|}
\hline & & Never & Rarely & Sometimes & Often & Very Often \\
\hline 1. & Unforeseen & .............. & $\ldots . .2$. & $\ldots \ldots 3 \ldots$ & ......4... & $\ldots .5 \ldots$ \\
\hline 2. & Platform & .............. & $\ldots .2 \ldots$ & $\ldots \ldots 3 \ldots$ & $\ldots . . .4$ & $\ldots . .$. \\
\hline 3. & Despised & ................. & $\ldots .2 \ldots$ & ......3..... & ......4... & ....5... \\
\hline 4. & Inhabit & ............... & $\ldots 2$. & $\ldots . .3 \ldots$ & ......4.. & $\ldots .5 \ldots$ \\
\hline 5. & Integrity & .............. & $\ldots 2$. & $\ldots . .3 \ldots$ & ....4.. & $\ldots 5 .$. \\
\hline
\end{tabular}

Note. In order to conserve space, only a sample of the first page of this questionnaire is provided. The remaining pages are identically formatted (with the exception of the instructions), and include the remaining study stimuli, in the order presented in Appendices A and B. 
Appendix E

Study 1 Imageability Questionnaire

\section{IMG}

Words differ in their capacity to arouse mental images of things or events. Some words arouse a sensory experience, such as a mental picture or sound, very quickly and easily, whereas other words may do so only with difficulty (i.e., after a long delay) or not at all. In this questionnaire you will be asked to rate a list of words on the ease or difficulty with which they arouse mental images.

Any word that in your opinion arouses a mental image (i.e., a mental picture, or sound, or other sensory experience) very quickly and easily should be given a high imagery rating (at the upper end of the numerical scale). Any word that arouses a mental image with difficulty or not at all should be given a low imagery rating (at the lower end of the numerical scale).

For example, think of the word "Buffalo". "Buffalo" would probably arouse an image relatively easily and would be rated as high imagery; "Relevant" would probably do so with difficulty and be rated as low imagery. Words can make you think of other words by association, but it is important that you only rate how easy it is to form a mental image of each specific word.

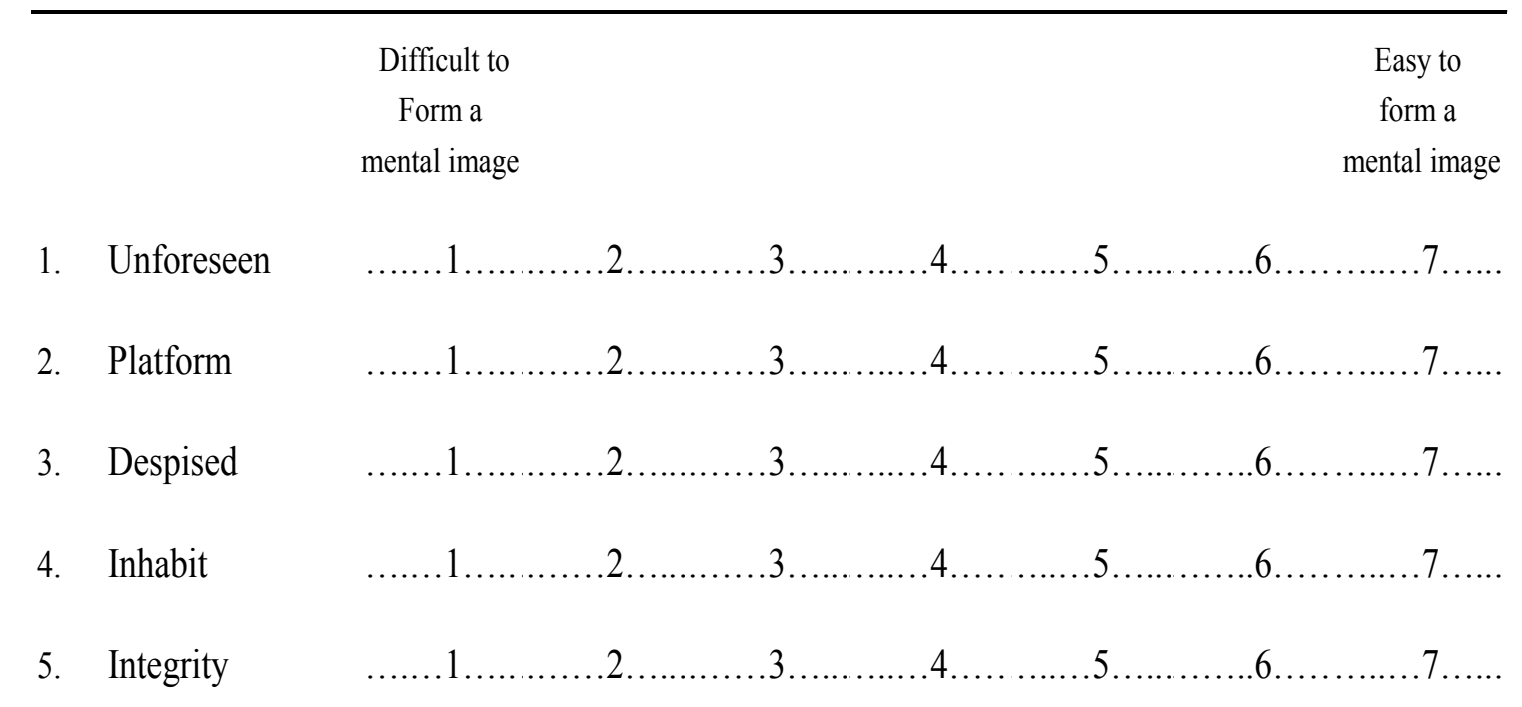

Note. In order to conserve space, only a sample of the first page of this questionnaire is provided. The remaining pages are identically formatted (with the exception of the instructions), and include the remaining study stimuli, in the order presented in Appendices A and B. 


\section{Appendix F}

Study 1 Uncertainty Questionnaire

\section{UN}

How much do the following words refer to uncertainty?

\begin{tabular}{ccccc} 
& \multicolumn{3}{c}{ Refers } \\
Does not refer & Refers a little & moderately & Refers a lot & Refers perfectly \\
to uncertainty & to uncertainty & to uncertainty & to uncertainty & to uncertainty
\end{tabular}

1. Unforeseen

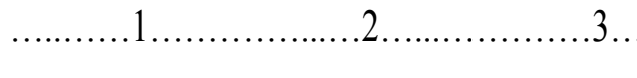

4 $.5 \ldots \ldots . .$.

2. Platform

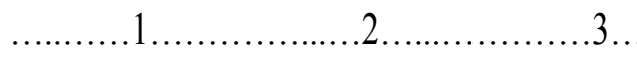

4 $.5 \ldots \ldots . . .$.

3. Despised

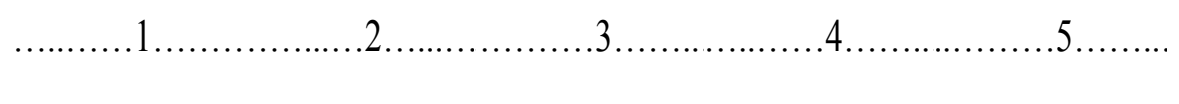

4. Inhabit

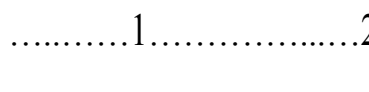

$2 \ldots \ldots \ldots \ldots \ldots \ldots$

4 $.5 \ldots \ldots . . .$.

5. Integrity

(.............................

$2 \ldots \ldots \ldots . . . . . . . . .3$ $4 \ldots \ldots \ldots \ldots \ldots \ldots \ldots \ldots \ldots$

Note. In order to conserve space, only a sample of the first page of this questionnaire is provided. The remaining pages are identically formatted (with the exception of the instructions), and include the remaining study stimuli, in the order presented in Appendices A and B. 
Appendix G

Study 1 Valence Questionnaire

\section{VAL}

How much of a negative or positive connotation do the following words have?

\begin{tabular}{|c|c|c|c|c|c|c|}
\hline & & $\begin{array}{c}\text { Very } \\
\text { negative }\end{array}$ & $\begin{array}{l}\text { A little } \\
\text { negative }\end{array}$ & $\begin{array}{c}\text { Neutral } \\
\text { (Neither positive } \\
\text { nor negative) }\end{array}$ & $\begin{array}{l}\text { A little } \\
\text { positive }\end{array}$ & $\begin{array}{c}\text { Very } \\
\text { positive }\end{array}$ \\
\hline 1. & Unforeseen & .........1..... & $\ldots .2 \ldots \ldots$ & .......3......... & $\ldots . . \ldots$. & $\ldots .5 \ldots$. \\
\hline 2. & Platform & $\ldots \ldots \ldots 1 \ldots \ldots$ & $\ldots .2 \ldots$ & $\ldots \ldots .3 \ldots \ldots$ & $\ldots 4 \ldots$ & $\ldots .5 \ldots$ \\
\hline 3. & Despised & $\ldots \ldots . .1 \ldots$ & $\ldots 2 \ldots$ & $\ldots \ldots . . .$. & $\ldots 4 \ldots$ & $\ldots .5 \ldots$ \\
\hline & Inhabit & $\ldots \ldots \ldots 1 \ldots$ & $\ldots 2 \ldots$ & $\ldots . .3 \ldots$ & $\ldots 4 \ldots$ & $\ldots . . .5$ \\
\hline & Integrity & $\ldots \ldots 1 \ldots$ &. .2 . & ...3. & .4 . & .5. \\
\hline
\end{tabular}

Note. In order to conserve space, only a sample of the first page of this questionnaire is provided. The remaining pages are identically formatted (with the exception of the instructions), and include the remaining study stimuli, in the order presented in Appendices A and B. 
Appendix $\mathrm{H}$

Study 1 General Information Questionnaire

\section{General Information}

Age:

Sex: $\quad$ Male

Female

\section{Education:}

University year: $\quad 1 \_\quad 2 \_$__ other

Field of study: Psychology Other (Please specify)

Status: full-time __ part-time

First Language: English

French

Other (please specify)

If English is not your first language, do you consider yourself fluent in English?

Yes

No

Race / Ethnicity: (check one)

African-American / Black / Caribbean Origin

Asian-American / Asian Origin / Pacific Islander

Latino-a / Hispanic

American Indian / Alaska Native / Aboriginal Canadian

European Origin / White

Bi-racial / Multi-racial

Middle Eastern

Other (Please Specify) 


\title{
Appendix I
}

\author{
Study 1 Consent form
}

\section{Consent form to Participate in Research}

This is to state that I agree to participate in a program of research conducted by Kylie Francis from the Anxiety Disorders Laboratory under the supervision of Dr. Michel Dugas, both of whom may be reached at 848-2424 ext. 2229.

\section{PURPOSE}

I have been informed that the purpose of this research is to obtain ratings of the concreteness, imageability, familiarity, uncertainty, and positive/negative characteristics of a series of words.

\section{PROCEDURE}

I have been informed that the study involves the following procedures: I will be asked to sign a consent form, and fill out a general information sheet. Then, I will be asked to complete a questionnaire package in which I will rate a set of words on their concreteness, imageability, familiarity, uncertainty, and positive/negative characteristics. The questionnaire should take 30 - 45 minutes to complete. My name will only appear on the consent form, and code numbers alone will be used to identify the questionnaires. The signed consent form will be stored separately from my responses to the questionnaires; all these documents will be kept under lock and key. I understand that my participation in the experiment, and the information I provide, are strictly confidential. As compensation for either participating in or observing this study, I will receive one course credit as outlined on the Psychology Department's Participant Pool website:

http://www-psychology.concordia.ca/Participants/index.html.

\section{CONDITIONS OF PARTICIPATION:}

- I understand that I am free to decline to participate in the experiment without negative consequences.

- I understand that I am free to withdraw my consent and discontinue my participation at any time without negative consequences.

- I understand that my participation in this study is confidential (i.e. the researcher will know, but will not disclose my identity).

- I understand that the data from this study may be published.

I HAVE CURRENTLY STUDIED THE ABOVE AND UNDERSTAND THIS AGREEMENT. I FREELY CONSENT AND AGREE TO PARTICIPATE IN THIS STUDY.

NAME (please print)

SIGNATURE

WITNESS SIGNATURE

DATE

If at any time you have questions about your rights as a research participant, please contact Adela Reid, Research Ethics and Compliance Officer, Concordia University, at 514-848-2424, x. 7481, or by email at Adela.Reid@Concordia.ca. 


\section{Appendix J}

\section{Study 1 Debriefing sheet}

\section{Debriefing sheet}

Title of Study: An investigation of information-processing biases related to worry: Pilot testing of stimulus word sets.

Principal Investigator: Kylie Francis, M. A, Doctoral candidate Supervisor: Michel J. Dugas, Ph. D, Associate Professor of Psychology

Summary of the research: Recent research suggests that individual differences in how people process information (information processing biases) may underlie the tendency to worry excessively. A study that will soon be carried out in the Anxiety Disorders Laboratory concerns information processing biases toward different types of words. In order to carry out this research, however, specific sets of words need to be selected for the research. The words that will be used need to be very similar (matched) on several characteristics: concreteness, imageability, familiarity, degree of uncertainty, and positive/negative valence. Matching is important because differences in these characteristics may make some words stand out more than others. For example, if a word is very unfamiliar, it may be more noticeable. Therefore, if these characteristics are not matched, and a participant processes one word differently than another, it would be impossible to know whether this was because of the word type, or because of differences in characteristics such as familiarity. The goal of this study was therefore to obtain participant ratings of a large set of words on the 5 characteristics. These ratings will be analyzed so that each word will have an average rating for concreteness, imageability, familiarity, uncertainty, and positive/negative valence. This will allow the researchers to select words that can be matched on these qualities for the upcoming information-processing study.

If you have any questions or concerns about this research, feel free to contact the Anxiety Disorders Laboratory, (514) 848-2424 ext, 2229. Email: anxiety@alcor.concordia.ca.

You may also contact the Chair of the Psychology Department Ethics Committee, Adam S. Radomsky, Ph.D., at ADAM.RADOMSKY@concordia.ca .

Or you may consult Adela Reid, Research Ethics and Compliance Officer, Concordia University, at 514-848-2424, x. 7481, or by email at Adela.Reid@Concordia.ca .

\section{Suggestions for further reading:}

MacLeod, C. \& Rutherford, E. (2004). Information-processing approaches: Assessing the selective functioning of attention, interpretation, and retrieval. In Cynthia L. Turk \& Richard G. Heimberg (Eds), Generalized anxiety disorder: Advances in research and practice (pp.109-142). New York, Guilford Press. 


\section{Appendix K}

Study 2 Consent form

\section{Consent form to Participate in Research}

This is to state that I agree to participate in a program of research conducted by Kylie Francis from the Anxiety Disorders Laboratory under the supervision of Dr. Michel Dugas, both of whom may be reached at 848-2424 ext. 2229.

\section{PURPOSE}

I have been informed that the purpose of this research is to investigate the relationship between how individuals process words, and how they react in different situations as measured by selfreport questionnaires.

\section{PROCEDURE}

I have been informed that the study involves the following procedures: I will be asked to sign a consent form. Next, I will be asked to view a series of words and carry out information-processing tasks. Finally, I will be asked to fill out a general information sheet and questionnaires that assess how people react in different types of situations. Participation should take approximately one hour. My name will only appear on the consent form, and code numbers alone will be used to identify the questionnaires. The signed consent form will not be kept with my responses to the questionnaires, and all these documents will be kept under lock and key. I understand that my participation in the experiment, and the information I provide, are strictly confidential. As compensation for either participating in or observing this study, I will receive one course credit as outlined on the Psychology Department's Participant Pool website:

http://www-psychology.concordia.ca/Participants/index.html.

\section{CONDITIONS OF PARTICIPATION:}

- I understand that I am free to decline to participate in the experiment without negative consequences.

- I understand that I am free to withdraw my consent and discontinue my participation at any time without negative consequences.

- I understand that my participation in this study is confidential (i.e. the researcher will know, but will not disclose my identity).

- I understand that the data from this study may be published.

I HAVE CURRENTLY STUDIED THE ABOVE AND UNDERSTAND THIS AGREEMENT. I FREELY CONSENT AND AGREE TO PARTICIPATE IN THIS STUDY.

NAME (please print)

\section{SIGNATURE}

\section{WITNESS SIGNATURE}

\section{DATE}

If at any time you have questions about your rights as a research participant, please contact Adela Reid, Research Ethics and Compliance Officer, Concordia University, at 514-848-2424, x. 7481, or by email at Adela.Reid@,Concordia.ca. 
Appendix L

Study 2 Familiarity Rating Sheet

\begin{tabular}{|c|c|c|}
\hline $\begin{array}{l}\text { Not at all } \\
\text { familiar }\end{array}$ & $\begin{array}{l}\text { Moderately } \\
\text { familiar }\end{array}$ & $\begin{array}{c}\text { Very } \\
\text { familiar }\end{array}$ \\
\hline 1 & 2 & 3 \\
\hline 1 & 2 & 3 \\
\hline 1 & 2 & 3 \\
\hline 1 & 2 & 3 \\
\hline 1 & 2 & 3 \\
\hline 1 & 2 & 3 \\
\hline 1 & 2 & 3 \\
\hline 1 & 2 & 3 \\
\hline 1 & 2 & 3 \\
\hline 1 & 2 & 3 \\
\hline 1 & 2 & 3 \\
\hline 1 & 2 & 3 \\
\hline 1 & 2 & 3 \\
\hline 1 & 2 & 3 \\
\hline 1 & 2 & 3 \\
\hline 1 & 2 & 3 \\
\hline
\end{tabular}

Note. In order to conserve space, only a sample of the first page of this questionnaire is provided. The remaining page is identical. 
Appendix M

Study 2 Recognition Sheet

\begin{tabular}{|c|c|c|}
\hline \multirow[t]{2}{*}{$\begin{array}{l}\text { For each word, please make a check under "NO" or "YES". } \\
\text { If you are not sure, follow your first guess. }\end{array}$} & \multicolumn{2}{|c|}{$\begin{array}{l}\text { Was this word } \\
\text { presented earlier? }\end{array}$} \\
\hline & NO & YES \\
\hline \multicolumn{3}{|l|}{ random } \\
\hline \multicolumn{3}{|l|}{ great } \\
\hline \multicolumn{3}{|l|}{ mocked } \\
\hline \multicolumn{3}{|l|}{ varying } \\
\hline \multicolumn{3}{|l|}{ tricky } \\
\hline \multicolumn{3}{|l|}{ customary } \\
\hline \multicolumn{3}{|l|}{ vague } \\
\hline \multicolumn{3}{|l|}{ mutilated } \\
\hline \multicolumn{3}{|l|}{ imprecise } \\
\hline \multicolumn{3}{|l|}{ mysterious } \\
\hline \multicolumn{3}{|l|}{ integrity } \\
\hline \multicolumn{3}{|l|}{ sickness } \\
\hline \multicolumn{3}{|l|}{ generous } \\
\hline \multicolumn{3}{|l|}{ enthusiastic } \\
\hline \multicolumn{3}{|l|}{ unsanitary } \\
\hline \multicolumn{3}{|l|}{ doubtful } \\
\hline \multicolumn{3}{|l|}{ puzzling } \\
\hline \multicolumn{3}{|l|}{ unlisted } \\
\hline \multicolumn{3}{|l|}{ inferior } \\
\hline \multicolumn{3}{|l|}{ actual } \\
\hline \multicolumn{3}{|l|}{ chance } \\
\hline \multicolumn{3}{|l|}{ confident } \\
\hline \multicolumn{3}{|l|}{ entire } \\
\hline \multicolumn{3}{|l|}{ ingredient } \\
\hline \multicolumn{3}{|l|}{ adored } \\
\hline \multicolumn{3}{|l|}{ delightful } \\
\hline \multicolumn{3}{|l|}{ rude } \\
\hline \multicolumn{3}{|l|}{ maybe } \\
\hline \multicolumn{3}{|l|}{ hesitation } \\
\hline rural & & \\
\hline reciprocal & & \\
\hline
\end{tabular}




\begin{tabular}{|c|c|c|}
\hline \multirow[t]{2}{*}{$\begin{array}{l}\text { For each word, please make a check under "NO" or "YES". } \\
\text { If you are not sure, follow your first guess. }\end{array}$} & \multicolumn{2}{|c|}{$\begin{array}{l}\text { Was this word } \\
\text { presented earlier? }\end{array}$} \\
\hline & $\mathrm{NO}$ & YES \\
\hline \multicolumn{3}{|l|}{ literal } \\
\hline \multicolumn{3}{|l|}{ talented } \\
\hline \multicolumn{3}{|l|}{ disapproval } \\
\hline \multicolumn{3}{|l|}{ ambiguous } \\
\hline \multicolumn{3}{|l|}{ worthy } \\
\hline \multicolumn{3}{|l|}{ uncertain } \\
\hline \multicolumn{3}{|l|}{ virus } \\
\hline \multicolumn{3}{|l|}{ universal } \\
\hline \multicolumn{3}{|l|}{ pleasant } \\
\hline \multicolumn{3}{|l|}{ lethal } \\
\hline \multicolumn{3}{|l|}{ modern } \\
\hline \multicolumn{3}{|l|}{ debatable } \\
\hline \multicolumn{3}{|l|}{ incompetent } \\
\hline \multicolumn{3}{|l|}{ questionable } \\
\hline \multicolumn{3}{|l|}{ caring } \\
\hline \multicolumn{3}{|l|}{ exterior } \\
\hline \multicolumn{3}{|l|}{ feverish } \\
\hline \multicolumn{3}{|l|}{ ignored } \\
\hline \multicolumn{3}{|l|}{ every } \\
\hline \multicolumn{3}{|l|}{ hated } \\
\hline \multicolumn{3}{|l|}{ irregular } \\
\hline \multicolumn{3}{|l|}{ unselfish } \\
\hline \multicolumn{3}{|l|}{ ridiculed } \\
\hline \multicolumn{3}{|l|}{ epidemic } \\
\hline \multicolumn{3}{|l|}{ good } \\
\hline \multicolumn{3}{|l|}{ admired } \\
\hline \multicolumn{3}{|l|}{ optimistic } \\
\hline \multicolumn{3}{|l|}{ geographical } \\
\hline methodical & & \\
\hline wonderful & & \\
\hline contaminated & & \\
\hline theoretical & & \\
\hline apparent & & \\
\hline
\end{tabular}

Note. The font and spacing of this questionnaire have been reduced to conserve space. 
Appendix N

Study 2 Debriefing sheet

\section{Debriefing sheet}

Title of Study: Working with words: An investigation of information-processing biases related to worry.

Principal Investigator: Kylie Francis, M. A, Doctoral candidate Supervisor: Michel J. Dugas, Ph. D, Associate Professor of Psychology

Summary of the research: Recent research suggests that individual differences in how people process information (information processing biases) may underlie the tendency to worry excessively. The goal of this study is to test information processing biases toward different types of information (in this study, words will be used). Participants are first asked to view words of different types and carry out information-processing tasks. After viewing the words, participants are asked to fill out a set of questionnaires. Based on their responses to the questionnaires, participants will be divided into two groups. These groups will be compared on how they processed different types of words. This research will help to clarify previous research in the area, which has been inconsistent. Furthermore, this research may suggest that certain types of words are useful for examining information-processing biases related to worry. Finally, the findings may have implications for the treatment of excessive worry. Specifically, researchers may investigate whether changing information processing biases during psychotherapy is an effective way to reduce chronic worry.

If you have any questions or concerns about this research, feel free to contact the Anxiety Disorders Laboratory, (514) 848-2424 ext, 2229. Email: anxiety@alcor.concordia.ca.

You may also contact Adam S. Radomsky, Ph.D., member of the Psychology Department Ethics Committee, at ADAM.RADOMSKY@concordia.ca .

Or you may consult Adela Reid, Research Ethics and Compliance Officer, Concordia University, at 514-848-2424, x. 7481, or by email at Adela.Reid@Concordia.ca .

\section{Suggestions for further reading:}

MacLeod, C. \& Rutherford, E. (2004). Information-processing approaches: Assessing the selective functioning of attention, interpretation, and retrieval. In Cynthia L. Turk \& Richard G. Heimberg (Eds), Generalized anxiety disorder: Advances in research and practice (pp.109-142). New York, Guilford Press. 


\section{Appendix O \\ Intolerance of Uncertainty Scale (IUS)}

\section{IUS}

You will find below a series of statements which describe how people may react to the uncertainties of life. Please use the scale below to describe to what extent each item is characteristic of you. Please circle a number ( 1 to 5 ) that describes you best.

\begin{tabular}{lccc}
\hline & $\begin{array}{c}\text { Not at all } \\
\text { characteristic } \\
\text { of me }\end{array}$ & $\begin{array}{c}\text { Somewhat } \\
\text { characteristic } \\
\text { of me }\end{array}$ & $\begin{array}{c}\text { Entirely } \\
\text { characteristic } \\
\text { of me }\end{array}$ \\
1. Uncertainty stops me from & & & of
\end{tabular}
having a firm opinion.

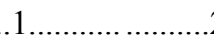
$.5 \ldots \ldots \ldots \ldots$

2. Being uncertain means that a person is disorganized.

.1 . 2.

4 $5 \ldots \ldots \ldots$

3. Uncertainty makes life intolerable.

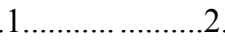
$2 \ldots \ldots \ldots \ldots \ldots \ldots . . . . . . . .$. 4 .5

4. It's unfair not having any guarantees in life. $\ldots 1 \ldots \ldots \ldots \ldots \ldots \ldots . . . .2$

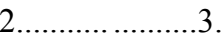

. .4 . .5 .

5. My mind can't be relaxed if I don't know what will happen tomorrow.

$1 \ldots \ldots \ldots \ldots \ldots \ldots . . .2$

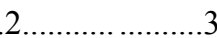

.4 $.5 \ldots \ldots \ldots$

6. Uncertainty makes me uneasy, anxious, or stressed.

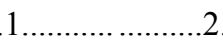

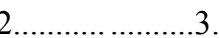
4.

7. Unforeseen events upset me greatly.

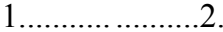

. .4 . 5.

8. It frustrates me not having all the information I need. .1. 2. .3. . .4 .

9. Uncertainty keeps me from living a full life. . .1 ...2. 3 4

10. One should always look ahead so as to avoid surprises. 2 3. . .4 . .5

11. A small unforeseen event can spoil everything, even with the best of planning. $1 .$. .2 . .3. . .4 . .5

12. When it's time to act, uncertainty paralyses me.

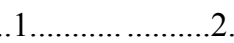
3. 4 5.

13. Being uncertain means that I am not first rate. ..1. 2 3. 4 
14. When I am uncertain, I can't go forward. $\ldots 1$

2 3. .4 .5.

15. When I am uncertain I can't function very well.

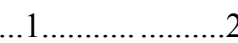

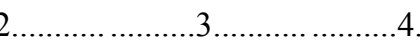
$.5 \ldots \ldots \ldots . . . .$.

16. Unlike me, others always seem to know where they are going with their lives.

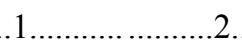
$2 \ldots \ldots \ldots \ldots$ 4 5.

17. Uncertainty makes me vulnerable, unhappy, or sad. 2 .3. 4 . $.5 \ldots \ldots \ldots \ldots$

18. I always want to know what the future has in store for me. .2 3. .4 $5 \ldots$

19. I can't stand being taken by surprise. $\ldots 1$ 2. ..3. $\ldots 4$.

20. The smallest doubt can stop me from acting.

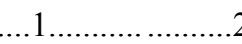

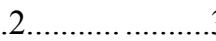

$3 \ldots \ldots \ldots \ldots \ldots \ldots . . . . . . .$. $5 \ldots \ldots \ldots$

21. I should be able to organize everything in advance.

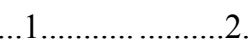
.3. ...4

22. Being uncertain means that I lack confidence.

$1 \ldots \ldots \ldots$ .................... 4

23. I think it's unfair that other people seem sure about their future.

.1. 2. 3. .4. .5.

24. Uncertainty keeps me from sleeping soundly. $\ldots 1$ . .2 . .3. $\ldots 4$......... 5

Origianl French Version: Freeston, M.H., Rhéaume, J., Letarte, H., Dugas, M.J., \& Ladouceur, R. (1994): Why do people worry? Personality and Individual Differences, 17 (6), 791-802.

English Version: Buhr, K., Dugas, M. J. (2002). The intolerance of uncertainty scale: psychometric properties of the English version. Behavior Research and Therapy, 40, 931-945.

Note. The font and spacing of this questionnaire have been reduced to conserve space. In the version participants completed, scale anchors appeared at the top of every page. 


\section{Appendix P}

\section{The Worry and Anxiety Questionnaire (WAQ)}

\section{WAQ}

1. What subjects do you worry about most often?

a)

b)

c)

For the following items, please circle the corresponding number (0 to 8).

\section{Do your worries seem excessive or exaggerated?}

Not at all excessive .0 . $0 \ldots \ldots \ldots \ldots . . .1$ $1 \ldots \ldots \ldots \ldots . . .2$
Moderately excessive

.4 d)

e)

f)

Totally excessive

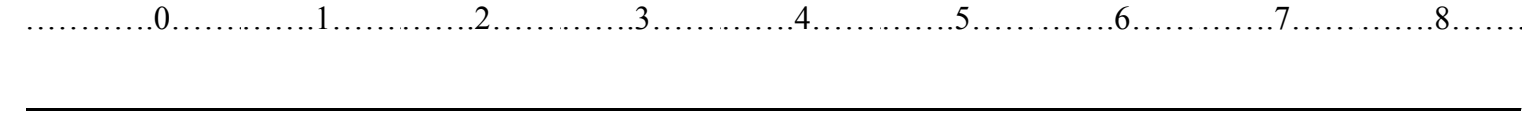

\section{Over the past six months, how many days have you been bothered by excessive worry?}

Never

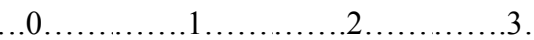
$3 \ldots \ldots \ldots . . . .4$ $4 \ldots \ldots \ldots \ldots . . . .5$ ..6. ..7. $8 \ldots \ldots$

4. Do you have difficulty controlling your worries? For example, when you start worrying about something, do you have difficulty stopping?

$\begin{array}{ccc}\text { No } & \text { Moderate } & \text { Extreme } \\ \text { difficulty } & \text { difficulty } & \text { difficulty }\end{array}$

. .2

3.

$. .4 \ldots \ldots \ldots \ldots . . .5$

..6. ..7. . $8 \ldots \ldots$ 
5. Over the past six months, to what extent have you been disturbed by the following sensations when you were worried or anxious? Rate each sensation by circling a number ( 0 to 8$)$.

a) Restlessness or feeling keyed up or on edge.

Not at all

Moderately

Very

Severely

$0 \ldots \ldots \ldots \ldots 1 \ldots \ldots \ldots . . . . . .$.

3.

$4 \ldots \ldots \ldots . .5$

6.

$8 \ldots \ldots$

b) Being easily fatigued.

Not at all

Moderately

Very

Severely

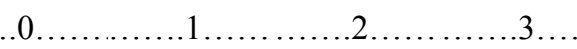

. .4.

. .5

66

.7

$8 \ldots \ldots$

c) Difficulty concentrating or mind going blank. $\begin{array}{lcc} & \text { Very } \\ \text { Not at all } & \text { Moderately } & \text { Severely }\end{array}$

$.0 \ldots \ldots \ldots \ldots 1 \ldots \ldots \ldots \ldots 2 \ldots \ldots \ldots \ldots . \ldots \ldots \ldots \ldots 4 \ldots \ldots \ldots \ldots .5 \ldots \ldots \ldots 6 \ldots \ldots \ldots 7 \ldots \ldots \ldots \ldots . \ldots \ldots$

d) Irritability.

Not at all

Moderately

.0 $1 \ldots \ldots \ldots . . .2$. 3. $4 \ldots \ldots \ldots \ldots$ ..6. .7 $8 \ldots \ldots$

e) Muscle tension.

Not at all

Moderately

Very Severely

.0 2 3. 4 .5 6. .7 . $.8 \ldots$

f) Sleep disturbance (difficulty falling or staying asleep, or restless unsatisfying sleep).

Not at all Moderately

0 . .1 .2 3

.4 5 . .6 . .7 $8 \ldots \ldots$

6. To what extent does worry or anxiety interfere with your life? For example, your work, social activities, family life, etc.?

Not at all

Moderately 2.

. .4 5 . .6. .7 $. .8 \ldots . .$.

French Version: Dugas, M. J., Freeston, M. H., Provencher, M. D., Lachance, S., Ladouceur, R., \& Gosselin, P. (2001). Le Questionnaire sur l'inquiétude et l'anxiété: Validation dans les échantillions non cliniques et cliniques [The Worry and Anx Questionnaire: Validation in nonclinical and clinical samples]. Journal de Thérapie Comportementale et Cognitive, 17, 31-

Note. The font and spacing of this questionnaire have been reduced to conserve space. 


\section{Appendix Q}

Subjective Units of Distress Scale (SUDS)

\section{SUDS}

1. On a scale from 0 to 100 , where 0 represents no anxiety and 100 represents extreme anxiety, what is your current level of anxiety?

Level of anxiety :

2. On a scale from 0 to 100 , where 0 represents no sadness and 100 represents extreme sadness, what is your current level of sadness?

Level of sadness :

3. On a scale from 0 to 100 , where 0 represents no irritability and 100 represents extreme irritability, what is your current level of irritability?

Level of irritability :

4. On a scale from 0 to 100 , where 0 represents an absence of well-being and 100 represents an extreme level of well-being, what is your current level of wellbeing?

Level of well-being :

5. On a scale from 0 to 100 , where 0 represents no fatigue and 100 represents extreme fatigue, what is your current level of fatigue?

Level of fatigue :

Dugas, M. J., Koerner, N., Heinecke, N. (2006). Concordia University, Anxiety Disorders Laboratory 
Appendix R

Study 2 General Information Sheet

General Information

Age:

Sex: $\quad$ Male __ Female

Education:

Highest Level Completed

Primary school CEGEP Bachelor's degree

Secondary school __ Certificate

Master's/Doctorate

Field of study: Psychology __ Other (Please specify)

Currently a student? No ___ Yes

IF currently a student: full-time ___ part-time

Work:

Status: full-time __ part-time __ not working

First Language:

English __ French___ Other (please specify)

$\underline{\text { Race / Ethnicity: (check one) }}$

African-American / Black / Caribbean Origin

Asian-American / Asian Origin / Pacific Islander

Latino-a / Hispanic

American Indian / Alaska Native / Aboriginal Canadian

European Origin / White

Bi-racial / Multi-racial

Middle Eastern

Other (Please Specify)

Civil Status: (check one)

Single

Civil Union

Married

Separated/Divorced

Widow/Widower

Note. The font and spacing of this questionnaire have been reduced to conserve space. 


\section{Appendix S}

Study 2: Hierarchical Regression with Sex, Social Desirability, Mood, and Intolerance of Uncertainty Predicting Recall for Neutral Words

\begin{tabular}{|c|c|c|c|c|c|}
\hline Predictor & $\Delta R^{2}$ & $B$ & $S E B$ & $\beta$ & $95 \% \mathrm{CI}$ \\
\hline Step 1 & .06 & & & & \\
\hline Constant & & 1.22 & .37 & & {$[.48,1.96]$} \\
\hline Sex & & -.26 & .37 & -.08 & {$[-1.00, .48]$} \\
\hline SDS & & .03 & .05 & .07 & {$[-.07, .13]$} \\
\hline SUDSanx & & .01 & .01 & .24 & {$[-.00, .03]$} \\
\hline SUDSirr & & -.01 & .01 & -.10 & {$[-.02, .01]$} \\
\hline
\end{tabular}

Step 2

.04

$\begin{array}{lcccc}\text { Constant } & .39 & .60 & & {[-.80,1.59]} \\ \text { Sex } & -.22 & .37 & -.07 & {[-.95, .51]} \\ \text { SDS } & .05 & .05 & .12 & {[-.05, .16]} \\ \text { SUDSanx } & .01 & .01 & .22 & {[-.00, .02]} \\ \text { SUDSirr } & -.01 & .01 & -.12 & {[-.02, .01]} \\ \text { IUS } & .01 & .01 & .20 & {[-.00, .03]}\end{array}$

Note. $n=86 . \mathrm{CI}=$ confidence interval for $B . \mathrm{SDS}=$ Social Desirability Scale; $\mathrm{SUDS}=$ Subjective Units of Distress: anx = anxiety, irr = irritability; IUS = Intolerance of Uncertainty Scale. 


\section{Appendix T}

Study 2: Hierarchical Regression with Sex, Social Desirability, Mood, and Intolerance of Uncertainty Predicting Recall for Uncertain Words

\begin{tabular}{|c|c|c|c|c|c|}
\hline Predictor & $\Delta R^{2}$ & $B$ & $S E B$ & $\beta$ & $95 \% \mathrm{CI}$ \\
\hline Step 1 & .03 & & & & \\
\hline Constant & & 2.11 & .41 & & {$[1.30,2.92]$} \\
\hline Sex & & -.18 & .41 & -.05 & {$[-.99, .63]$} \\
\hline SDS & & -.05 & .06 & -.10 & {$[-.16, .07]$} \\
\hline SUDSanx & & .01 & .01 & .10 & {$[-.01, .02]$} \\
\hline SUDSirr & & -.01 & .01 & -.22 & {$[-.03, .00]$} \\
\hline
\end{tabular}

Step 2

.01

$\begin{array}{lcccc}\text { Constant } & 2.53 & .67 & & {[1.20,3.86]} \\ \text { Sex } & -.20 & .41 & -.06 & {[-1.01, .61]} \\ \text { SDS } & -.06 & .06 & -.12 & {[-.17, .06]} \\ \text { SUDSanx } & .01 & .01 & .11 & {[-.01, .02]} \\ \text { SUDSirr } & -.01 & .01 & -.21 & {[-.03, .00]} \\ \text { IUS } & -.01 & .01 & -.09 & {[-.03, .01]}\end{array}$

Note. $n=86 . \mathrm{CI}=$ confidence interval for $B . \mathrm{SDS}=$ Social Desirability Scale; $\mathrm{SUDS}=$ Subjective Units of Distress: anx = anxiety, irr = irritability; IUS = Intolerance of Uncertainty Scale. 


\section{Appendix U}

Study 2: Hierarchical Regression with Sex, Social Desirability, Mood, and Intolerance of Uncertainty Predicting Recall for Threat Words

\begin{tabular}{|c|c|c|c|c|c|}
\hline Predictor & $\Delta R^{2}$ & $B$ & $S E B$ & $\beta$ & $95 \% \mathrm{CI}$ \\
\hline Step 1 & .05 & & & & \\
\hline Constant & & 1.49 & .37 & & {$[.77,2.20]$} \\
\hline Sex & & -.20 & .36 & -.06 & {$[-.92, .52]$} \\
\hline SDS & & -.01 & .05 & -.01 & {$[-.11, .09]$} \\
\hline SUDSanx & & .01 & .01 & .27 & {$[.00, .03]$} \\
\hline SUDSirr & & -.01 & .01 & -.14 & {$[-.02, .01]$} \\
\hline
\end{tabular}

Step 2

.00

$\begin{array}{lcccc}\text { Constant } & 1.56 & .60 & & {[.37,2.75]} \\ \text { Sex } & -.20 & .37 & -.06 & {[-.93, .53]} \\ \text { SDS } & -.01 & .05 & -.02 & {[-.11, .10]} \\ \text { SUDSanx } & .01 & .01 & .27 & {[.00, .03]} \\ \text { SUDSirr } & -.01 & .01 & -.14 & {[-.02, .01]} \\ \text { IUS } & -.00 & .01 & -.02 & {[-.02, .02]}\end{array}$

Note. $n=86 . \mathrm{CI}=$ confidence interval for $B . \mathrm{SDS}=$ Social Desirability Scale; $\mathrm{SUDS}=$ Subjective Units of Distress: anx = anxiety, irr = irritability; IUS = Intolerance of Uncertainty Scale. 


\section{Appendix V}

Study 2: Hierarchical Regression with Sex, Social Desirability, Mood, and Intolerance of Uncertainty Predicting Recall for Positive Words

\begin{tabular}{|c|c|c|c|c|c|}
\hline Predictor & $\Delta R^{2}$ & $B$ & $S E B$ & $\beta$ & $95 \% \mathrm{CI}$ \\
\hline Step 1 & .05 & & & & \\
\hline Constant & & 2.63 & .41 & & {$[1.81,3.45]$} \\
\hline Sex & & -.64 & .41 & -.17 & {$[-1.45, .18]$} \\
\hline SDS & & -.06 & .06 & -.12 & {$[-.17, .05]$} \\
\hline SUDSanx & & .01 & .01 & .18 & {$[-.01, .03]$} \\
\hline SUDSirr & & -.01 & .01 & -.18 & {$[-.03, .01]$} \\
\hline
\end{tabular}

Step 2

.02

$\begin{array}{lcccc}\text { Constant } & 1.89 & .67 & & {[.56,3.21]} \\ \text { Sex } & -.60 & .41 & -.16 & {[-1.41, .22]} \\ \text { SDS } & -.04 & .06 & -.08 & {[-.16, .07]} \\ \text { SUDSanx } & .01 & .01 & .17 & {[-.01, .02]} \\ \text { SUDSirr } & -.01 & .01 & -.20 & {[-.03, .00]} \\ \text { IUS } & .01 & .01 & .16 & {[-.01, .03]}\end{array}$

Note. $n=86 . \mathrm{CI}=$ confidence interval for $B . \mathrm{SDS}=$ Social Desirability Scale; $\mathrm{SUDS}=$ Subjective Units of Distress: anx = anxiety, irr = irritability; IUS = Intolerance of Uncertainty Scale. 
Appendix W

Study 3 Telephone Screening

\section{PHONE INTERVIEW - Study 3}

"As I mentioned, the first step in this study is a 20-minute phone screening. That's because we're looking for people who meet specific criteria for our research. So, l'll be asking you some questions, and filling out your answers on a form as we talk. Everything that you tell me is completely confidential, and the form will be stored in a locked cabinet. If you meet the requirements, you'll be invited to come to the lab for the study. However, based on your answers, it may be that you are not eligible for the study. In that case I won't be able to invite you to take part in the experiment. Are you still willing to continue with the phone screening?"

Interviewer name:

Participant name:

Date:

Age: (must be 18-65)

First Language ENGLISH

OTHER

Defined as the language they spoke at home on a daily basis before the age of 5 (EXCLUDE)

Normal or corrected to normal vision? YES

NO (EXCLUDE)

Glasses or contacts are fine as long as their vision is normal while wearing them

Normal or corrected to normal hearing? YES NO (EXCLUDE)

Are you currently taking any medication? If YES, What medication? How long have you been taking it at that dose? Establish exact dose per day, and duration. If they take anxiety medications as needed (PRN), establish frequency \& stability. To be included in the study, medication \& dose should have been stable for 4 weeks (though a reduction is less problematic than an increase).

MEDICATION STABLE (or none) YES_NO $\quad$ N_ If NO, but medication would meet criteria for stability in near future, ask whether participant is willing to wait, maintaining a stable dose, in order to participate. Also inform them that a requirement for the study is not to take any drugs, or drink alcohol, or take any NEW medications (including cold medication) the night before or the day of the study. Verify that this is ok with them.

Have you ever been diagnosed as having a reading disability or speech impediment? YES (EXCLUDE) NO

Have you ever been diagnosed with schizophrenia, bipolar disorder, or any other mental illness? YES (EXCLUDE) NO

If YES: Specify:

Can you can tell me what motivated you to call about the study? 
GAD

1a. In general, what are some of the things you tend to worry about?

a)

b)

c)

d)

1b. Do your worries seem excessive or exaggerated to you?

YES _ NO N

IF you are unsure of participant's report: Why do you think they [are/are not] exaggerated?

1c. Do you have trouble controlling your worries? For example, once you start to worry, do you have difficulty stopping? If unclear, ask whether worries persist even when he/she does not want to worry; if they do, check YES.

YES N NO

**If NO to $1 b$ and $1 c$ skip to DEPRESSION section. If YES to $1 b$ or $1 c$ continue to question 2.

2. Recently, have you been bothered by worries more days than not? i.e., in the last few months. If participant is unsure, ask whether he/she is worried most of the time, half the time, or less than half the time. If most or more than half of the time, check YES.

$$
\text { YES _ NO N }
$$

3. On a typical day during the past month, how much time did you spend worrying? Can be reported in mins, hrs, or \%, as preferred; specify which when recording response

4. During the past six months, have you been bothered by:
a) Feeling agitated, over-excited or "on-edge
YES
NO
b) Fatigue
YES
NO
c) Difficulties concentrating, mind going blank;
YES
NO
d) Irritability
YES
NO
e) Muscle tension
YES
NO
f) Sleep problems
YES
NO

If the person endorses at least 3 of these symptoms, he/she meets the somatic criteria. 
5. Do your worries or anxiety interfere with your life (your work, social activities, family, etc)? If unclear, ask whether worry and anxiety lead to avoidance of activities; problems with friends or family, difficulties at work, inability to relax or enjoy things, emotional distress, etc.

$$
\text { YES _ _ NO___ If YES, the person meets interference criteria. }
$$

6. For how long have worries or anxiety been a problem for you? [Would you say you've always been a worrier? When did you start to notice that?] i.e., number of months or years since worries became a problem. Can ask in terms of development (childhood, teens, 20s, etc.), life events (CEGEP, University, jobs, birth of children), or particular age when worries first became a problem.

YEARS

MONTHS

GAD :

NOT PROBABLE

PROBABLE

OTHER ANXIETY DISORDER:

NOT PROBABLE

PROBABLE

If other anxiety disorder probable, specify:

\section{DEPRESSION}

1a. Recently, have you been feeling depressed, sad, or empty? i.e., in the past 2 weeks.
Depressed:
YES
NO

Lately, have you lost interest or pleasure in almost all of your usual activities? Loss of interest $\quad$ YES __ NO

1b. Recently, have other people commented that you seem sad or down?
Depressed:
YES
$\mathrm{NO}$

Has anyone recently said you seem less interested in things you usually enjoy?

Loss of interest

YES $\mathrm{NO}$

**If NO to $1 a$ and $1 b$ skip to question 5a. If YES to either $1 a$ or $1 b$ continue to question 2.

2. Have you been feeling [depressed/lacking interest] nearly every day over the past two weeks?

$\begin{array}{lll}\text { Depressed: } & \text { YES } & \text { NO } \\ \text { Loss of interest } & \text { YES } & \text { NO }\end{array}$

3. Over the past $\mathbf{2}$ weeks, have you:

Read symptoms below; if YES ask whether present most days (if so, check 'Most days').

\begin{tabular}{|l|c|c|c|}
\hline & \multicolumn{2}{|l|}{} & Most days? \\
\hline 3a. & $\begin{array}{c}\text { Lost or gained a significant amount of weight? e.g. 5\% of } \\
\text { body weight within a month. Had a major decrease or } \\
\text { increase in appetite? }\end{array}$ & YES NO & \\
\hline 3b. & $\begin{array}{c}\text { Had sleep problems? insomnia, interrupted sleep, } \\
\text { oversleeping }\end{array}$ & YES NO & \\
\hline 3c. & $\begin{array}{c}\text { Felt so agitated you couldn't sit still, or so slowed down it } \\
\text { was hard to move or carry on a conversation? }\end{array}$ & YES NO & \\
\hline 3d. & Been fatigued, tired, or lacking energy? & YES NO & \\
\hline 3e. & $\begin{array}{c}\text { Have you felt extremely guilty, worthless, or blamed } \\
\text { yourself for things? worthlessness or excessive, } \\
\text { inappropriate guilt. }\end{array}$ & YES NO & \\
\hline $3 f$. & $\begin{array}{c}\text { Felt your thinking was slowed down, or you had trouble } \\
\text { concentrating? Had trouble making decisions? }\end{array}$ & YES NO & \\
\hline $3 \mathrm{Bg}$. & $\begin{array}{c}\text { Had thoughts about death or hurting yourself? Have you } \\
\text { thought about suicide? }\end{array}$ & YES NO & \\
\hline
\end{tabular}


3h. If symptoms seem severe: How have these symptoms of depression interfered with your life (e.g. daily routine, job, social activities)? How much do they bother you?

DEPRESSION: NOT PROBABLE

PROBABLE

(EXCLUDE)

**EVEN IF EXCLUDED:

IF PERSON RESPONDED YES TO 3g, CONTINUE TO QUESTION 4.

If person responded NO to $\mathbf{3 g}$, check "LEVEL O" in SUICIDALITY section, and continue with question 5. Offer participant referral/number for help with depression if suicidality is not present.

4. IF person responded YES to 3g: What kind of thoughts of death or suicide have you had? Assess: Concreteness of ideas; presence of specific plan; access to method for carrying out plan; specific timeline for plan; ability to state reasons for living. Note the difference between actual suicidal ideation and self-harm obsessions. In Self-harm obsessions, thoughts about death or harming oneself are intrusive in that the person does not want to have them (egodystonic). The person may fear that because they are having these thoughts, they might commit suicide without actually wanting to.

5a. ASK ONLY IF NOT CURRENTLY DEPRESSED: It sounds like your mood is fairly good right now; have you had any thoughts about death or harming yourself? Check level below

SUICIDALITY Based on the person's description p. $\underline{4-6}$, check one of the 4 LEVELS below: LEVEL O: No current suicidal ideation LEVEL 1: Vague thoughts about suicide, but no plan LEVEL 2: Fuzzy plan (i.e. would take pills, but don't know specifically what pills, or how many are needed, or where to get these pills etc...) LEVEL 3: Clear plan, but no intention or timeline of when it will take place LEVEL 4: Clear plan and clear intention of when it will take place

5b. Have you ever acted on thoughts about suicide, or attempted suicide in the past? If YES, How long ago? ATTEMPT 0: Never attempted suicide ATTEMPT 1: Suicide attempted more than 2 years ago ATTEMPT 2: Suicide attempt made within the past 2 years

Based on the responses above, check one of the categories below:

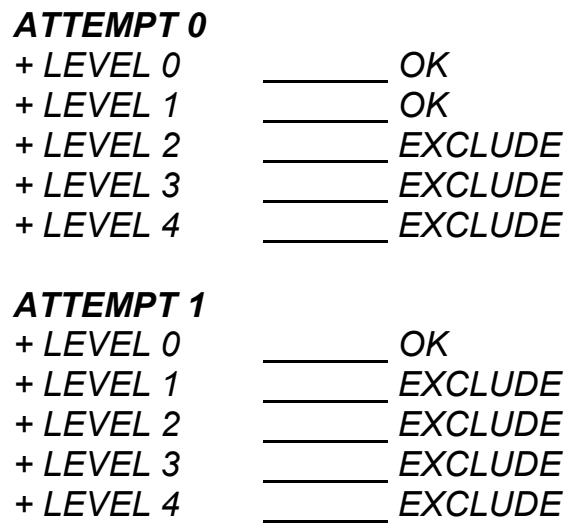


ATTEMPT 2

$+A N Y L E V E L=E X C L U D E$

IF EXCLUDED AT LEVEL 3 or LEVEL 4: Put participant on hold; ask Kylie to take the call. Any other level of exclusion: Confirm absence of immediate intention; give referral/number for helpline, information about nearest hospital emergency, and any other information participant requests.

\section{PROBLEM GAMBLING}

\section{Currently, do you gamble?}

$$
\text { YES _ NO N }
$$

If YES, Has gambling caused you financial problems? Has it caused problems with your friends or family, school or work? Have you tried to stop gambling but have been unable to?

PROBLEM GAMBLING: - (EXCLUDE)

\section{NOT PROBABLE}

If participant is excluded, offer referral/number for helpline.

\section{SUBSTANCE ABUSE}

1. Currently, how much alcohol do you drink? Specify number, type, and amount of alcoholic beverage used and indicate time period; e.g., "three 12 oz. beers per week.")

If alcohol use seems excessive: Has your drinking created problems at school or work? With your friends or family? Have you tried to stop drinking but have been unable to

2. Besides alcohol, do you currently use other substances such as marijuana, cocaine, or other recreational drugs?

$$
\text { YES _ NO }
$$

If YES, What do you use? Typically, how much and how often?

If use seems excessive: Has it caused problems at school or work? With your friends or family? Have you tried to stop but have been unable to?

\section{SUBSTANCE ABUSE}

NOT PROBABLE

PROBABLE

If participant is excluded, offer referral/number for helpline.

(EXCLUDE) 


\section{AT END OF INTERVIEW}

Thank participant; Inform them whether they are eligible for the study, based on their answers. If eligible, schedule them for testing, reminding them that they should not take any substances the day of, or the night before, testing. *Schedule on Gmail calendar.

OPTIONAL SECTION - ADMINISTER ONLY IF NEEDED / UNSURE

\section{NON-ORGANIC PSYCHOSIS}

Has there ever been a time when you had strange or unusual experiences such as:

a) Hearing or seeing things that other people didn't notice? YES

NO

b) Hearing voices or conversations when no one was around? YES

NO

c) Having visions that no one else saw?

YES

NO

d) Having the feeling that something odd was going on around you, that people were doing things to test you or antagonize or hurt you so that you felt you had to be on guard constantly?

YES

NO

If YES to any of the above, specify:

PSYCHOSIS: NOT PROBABLE

PROBABLE

(EXCLUDE)

If participant is excluded, offer referral/number for helpline.

Note. The font and spacing of this measure have been reduced to conserve space. 
Appendix X

Study 3 Calibration Sheet

\section{CALIBRATION}

\begin{tabular}{l|l|l|}
\multicolumn{2}{l}{$\mathbf{8 3} \mathbf{~ m s}$} & \multicolumn{1}{l}{ Correct? } \\
\cline { 2 - 3 } 1 & UNDO & \\
\cline { 2 - 3 } 2 & VERSE & \\
\cline { 2 - 3 } 3 & MEASURE & \\
\cline { 2 - 3 } 4 & NEUTRAL & \\
\cline { 2 - 3 } 5 & QUANTITY & \\
\cline { 2 - 3 } 6 & INFORMATION & \\
\cline { 2 - 3 } 7 & UNFOLD & \\
\cline { 2 - 3 } & \multicolumn{2}{|c}{ TOTAL $=$}
\end{tabular}

\begin{tabular}{|c|c|c|}
\hline & $67 \mathrm{~ms}$ & Correct? \\
\hline 1 & RECENT & \\
\hline 2 & ORIGIN & \\
\hline & ALPHABET & \\
\hline & UNLOCK & \\
\hline 5 & INTERVAL & \\
\hline 6 & EARLY & \\
\hline & INSTRUCTION & \\
\hline
\end{tabular}

\begin{tabular}{|c|c|c|}
\hline & $50 \mathrm{~ms}$ & Correct? \\
\hline 1 & MANY & \\
\hline 2 & PAST & \\
\hline 3 & ANSWER & \\
\hline 4 & UNDERAGED & \\
\hline 5 & INSIDE & \\
\hline 6 & MATHEMATICAL & \\
\hline 7 & SIMILAR & \\
\hline
\end{tabular}

\begin{tabular}{l|l|l|}
\multicolumn{1}{l}{$33 \mathbf{~ m s}$} & Correct? \\
\cline { 2 - 3 } 1 & UNIT & \\
\cline { 2 - 3 } 2 & SANDY & \\
\cline { 2 - 3 } 3 & IMPORT & \\
\cline { 2 - 3 } 4 & INSTALL & \\
\cline { 3 - 3 } 5 & SUBSTITUTE & \\
\cline { 2 - 3 } 6 & PRESENCE & \\
\cline { 2 - 3 } 7 & UNSWEETENED & \\
\cline { 2 - 3 } & \multicolumn{2}{|c|}{ TOTAL $=$} \\
\end{tabular}

Circle duration that is participant's WORST performance (fewest correct)

If two or more are equal, select the LOWER number (i.e., faster duration).

If $7,6,5$, or 4 correct. use one duration FASTER

If $\mathbf{3}$ or $\mathbf{2}$ correct

use SAME duration

If 1 or 0 correct

use one duration SLOWER

\begin{tabular}{|c|}
\hline $100 \mathrm{~ms}$ \\
\hline $83 \mathrm{~ms}$ \\
\hline $67 \mathrm{~ms}$ \\
\hline $50 \mathrm{~ms}$ \\
\hline $33 \mathrm{~ms}$ \\
\hline $17 \mathrm{~ms}$ \\
\hline
\end{tabular}

$$
\begin{array}{r}
\text { Select duration } \\
\text { for Wd ID task } \\
\text { (circle one) }
\end{array}
$$

Math start time:

Recall start time: (+ 5 mins) end time: (+ 5 mins) end time: 
Appendix Y

Study 3 Distractor Task
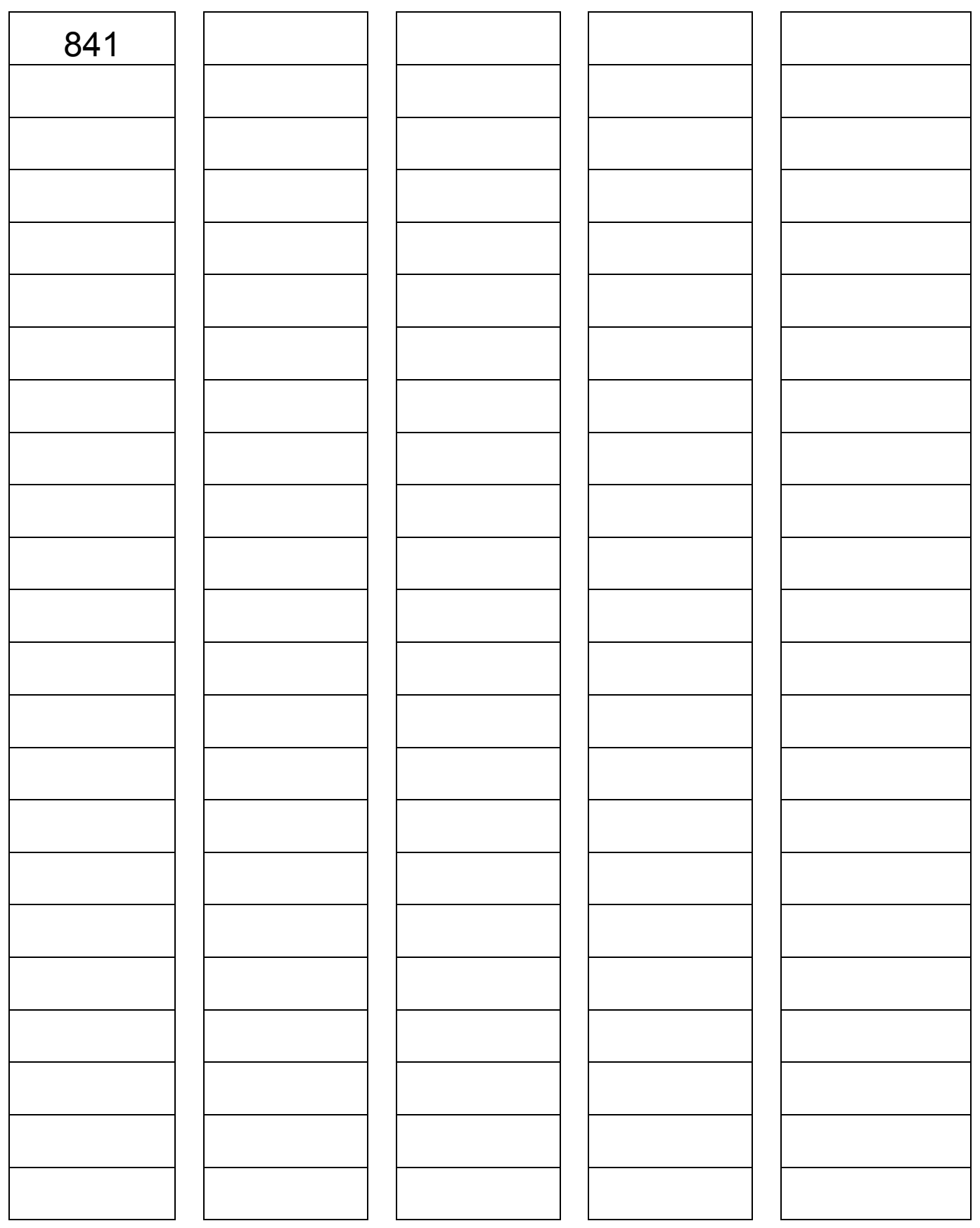
Appendix Z

Study 3 Debriefing sheet

\section{Debriefing sheet}

Title of Study: An investigation of information-processing biases related to worry.

Principal Investigator: Kylie Francis, M. A, Doctoral candidate

Supervisor: Michel J. Dugas, Ph. D, Associate Professor of Psychology

Summary of the research: Attempts to understand how people attend to, interpret, and remember information have led to a growing body of what is called 'information processing' research. Recently, researchers have proposed that specific informationprocessing biases may underlie the tendency to worry excessively. The goal of this study is to test information processing biases toward different types of information (in this study, words are used). Participants are first asked to view words of different types and carry out information-processing tasks. After viewing the words, participants are asked to fill out a set of questionnaires and take part in a brief interview. Based on their responses to the questionnaires and interview, participants will be divided into high and low worry groups. These groups will be compared on how they processed different types of words. This research will help to clarify previous research in the area, which has been inconsistent. Furthermore, this research may suggest that certain types of words can be effectively used to examine information-processing biases related to worry. Finally, the findings may have implications for the treatment of excessive worry. Specifically, researchers may investigate whether changing information processing biases during psychotherapy is an effective way to reduce chronic worry.

If you have any questions or concerns about this research, feel free to contact the Anxiety Disorders Laboratory, (514) 848-2424 ext, 2229. Email: anxiety@alcor.concordia.ca.

You may also contact Virginia Penhune, Ph.D., chair of the Psychology Department Ethics Committee, at penhune@alcor.concordia.ca.

Or you may consult Adela Reid, Research Ethics and Compliance Officer, Concordia University, at 514-848-2424, x. 7481, or by email at Adela.Reid@Concordia.ca .

\section{Suggestions for further reading:}

MacLeod, C. \& Rutherford, E. (2004). Information-processing approaches: Assessing the selective functioning of attention, interpretation, and retrieval. In Cynthia L. Turk \& Richard G. Heimberg (Eds), Generalized anxiety disorder: Advances in research and practice (pp.109-142). New York, Guilford Press. 


\title{
Appendix AA
}

The Stimulus Relevance Questionnaire (SRQ)

\section{Stimulus Relevance Questionnaire}

\begin{abstract}
Please carefully read the words below and rate each word on how relevant it is to you; In other words, rate how much each word corresponds to events or topics that are currently important to you (i.e. within the past month). Rate each word by circling the appropriate number, where:

$0=$ Not at all Relevant to Me $2=$ Moderately Relevant to Me 4 = Extremely Relevant to Me
\end{abstract}

Please make a rating for every word, even though the relevance of some words may not be readily apparent.

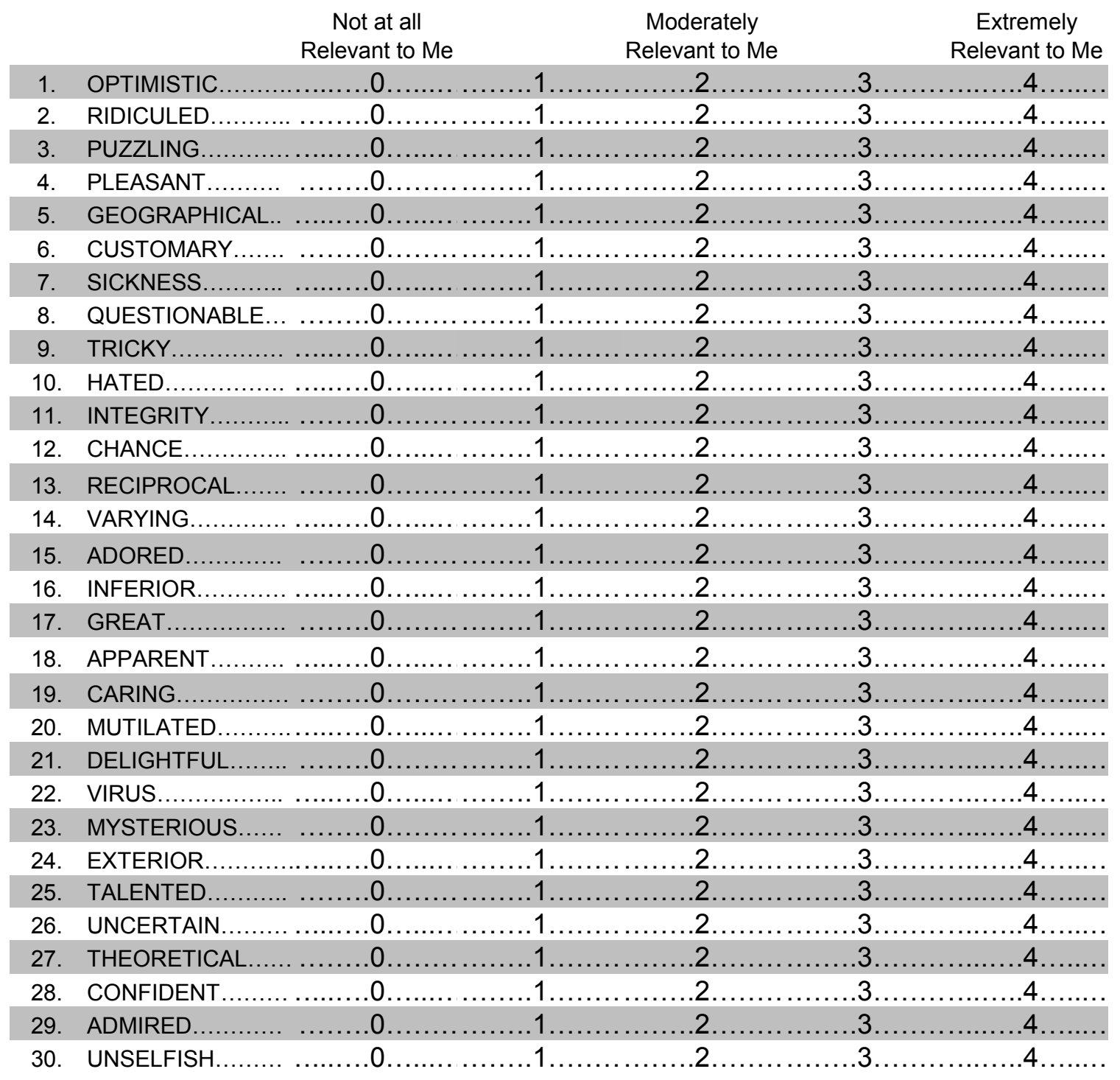




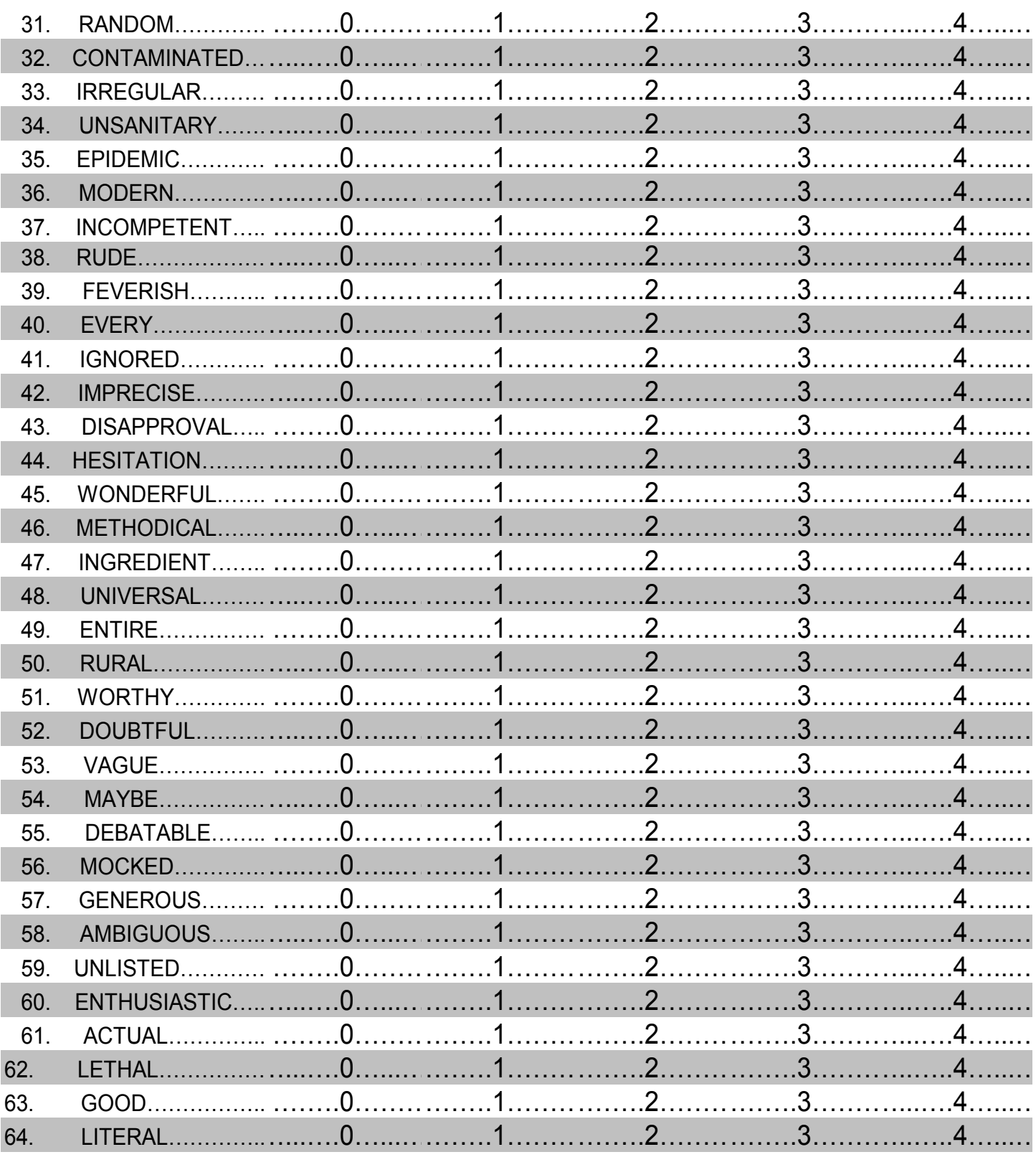

Note. The font and spacing of this questionnaire have been reduced to conserve space. In the version participants completed, scale anchors appeared at the top of every page. 
Appendix AB

Manipulation Check Form (MC)

\section{MC-3}

Please make a response by checking ONLY ONE of the boxes below. Even if you think more than one answer may be correct, please choose only one answer, the answer that seems most correct to you.

What do you think the purpose of the computer tasks was? (i.e., NOT the written questions)

To assess the speed of your perceptual ability when working with words

To measure your verbal ability

To measure how quickly you can co-ordinate auditory instructions, visual material, and verbal responses

To measure memory for different words

To assess how your vocabulary affects your perceptual speed when working with words Don't know / none of the above

Please briefly explain why you chose this answer (unless you checked "Don't know"): 


\section{Appendix AC}

Study 3: Hierarchical Multiple Regression with Demographics, Intolerance of Uncertainty, and Stimulus Relevance Predicting Recall of Neutral Words

\begin{tabular}{|c|c|c|c|c|c|}
\hline Predictor & $\Delta R^{2}$ & $B$ & $S E B$ & $\beta$ & $95 \% \mathrm{CI}$ \\
\hline Step 1 & .04 & & & & \\
\hline Constant & & .59 & .73 & & {$[-.86,2.03]$} \\
\hline Age & & -.02 & .01 & -.21 & {$[-.03, .00]$} \\
\hline Sex & & -.12 & .21 & -.06 & {$[-.53, .30]$} \\
\hline Vocab & & .02 & .03 & .08 & {$[-.04, .08]$} \\
\hline Step 2 & .01 & & & & \\
\hline Constant & & .46 & .74 & & {$[-1.02,1.93]$} \\
\hline Age & & -.02 & .01 & -.20 & {$[-.03, .00]$} \\
\hline Sex & & -.09 & .21 & -.04 & {$[-.51, .33]$} \\
\hline Vocab & & .02 & .03 & .06 & {$[-.04, .08]$} \\
\hline IUS & & .00 & .00 & .10 & {$[-.00, .01]$} \\
\hline Step 3 & .00 & & & & \\
\hline Constant & & .51 & .77 & & {$[-1.02,2.04]$} \\
\hline Age & & -.02 & .01 & -.20 & {$[-.03, .00]$} \\
\hline Sex & & -.08 & .21 & -.04 & {$[-.51, .35]$} \\
\hline Vocab & & .02 & .03 & .06 & {$[-.04, .08]$} \\
\hline IUS & & .00 & .00 & .10 & {$[-.00, .01]$} \\
\hline SRn & & -.00 & .01 & -.03 & {$[-.02, .01]$} \\
\hline
\end{tabular}

Note. $n=98 . \mathrm{CI}=$ confidence interval for $B$. Vocab $=$ Mill Hill Vocabulary Scale; IUS = Intolerance of Uncertainty Scale; SRn = stimulus relevance for neutral words. 


\section{Appendix AD}

Study 3: Hierarchical Multiple Regression with Demographics, Intolerance of Uncertainty, and Stimulus Relevance Predicting Recall of Uncertain Words

\begin{tabular}{|c|c|c|c|c|c|}
\hline Predictor & $\Delta R^{2}$ & $B$ & $S E B$ & $\beta$ & $95 \% \mathrm{CI}$ \\
\hline Step 1 & .02 & & & & \\
\hline Constant & & .96 & 1.01 & & {$[-1.04,2.96]$} \\
\hline Age & & -.01 & .01 & -.07 & {$[-.03 .02]$} \\
\hline Sex & & -.27 & .29 & -.10 & {$[-.85, .30]$} \\
\hline Vocab & & .02 & .04 & .05 & {$[-.06, .10]$} \\
\hline Step 2 & .02 & & & & \\
\hline Constant & & .70 & 1.02 & & {$[-1.32,2.72]$} \\
\hline Age & & -.01 & .01 & -.07 & {$[-.03, .02]$} \\
\hline Sex & & -.21 & .29 & -.08 & {$[-.79, .36]$} \\
\hline Vocab & & .01 & .04 & .02 & {$[-.07, .09]$} \\
\hline IUS & & .01 & .01 & .14 & {$[-.00, .02]$} \\
\hline Step 3 & .02 & & & & \\
\hline Constant & & .36 & 1.04 & & {$[-1.70,2.43]$} \\
\hline Age & & -.00 & .01 & -.02 & {$[-.03, .02]$} \\
\hline Sex & & -.28 & .29 & -.11 & {$[-.87, .30]$} \\
\hline Vocab & & .01 & .04 & .03 & {$[-.07, .09]$} \\
\hline IUS & & .00 & .01 & .05 & {$[-.01, .02]$} \\
\hline SRun & & .02 & .01 & .18 & {$[-.01, .04]$} \\
\hline
\end{tabular}

Note. $n=98 . \mathrm{CI}=$ confidence interval for $B$. Vocab $=$ Mill Hill Vocabulary Scale; IUS $=$ Intolerance of Uncertainty Scale; SRun = stimulus relevance for uncertain words. 


\section{Appendix AE}

Study 3: Hierarchical Multiple Regression with Demographics, Intolerance of Uncertainty, and Stimulus Relevance Predicting Recall of Threat Words

\begin{tabular}{|c|c|c|c|c|c|}
\hline Predictor & $\Delta R^{2}$ & $B$ & $S E B$ & $\beta$ & $95 \% \mathrm{CI}$ \\
\hline Step 1 & .04 & & & & \\
\hline Constant & & -.15 & .70 & & {$[-1.54,1.25]$} \\
\hline Age & & -.01 & .01 & -.06 & {$[-.02, .01]$} \\
\hline Sex & & -.33 & .20 & -.17 & {$[-.73, .07]$} \\
\hline Vocab & & .04 & .03 & .14 & {$[-.02, .09]$} \\
\hline Step 2 & .01 & & & & \\
\hline Constant & & -.24 & .72 & & {$[-1.66,1.18]$} \\
\hline Age & & -.01 & .01 & -.06 & {$[-.02, .01]$} \\
\hline Sex & & -.31 & .20 & -.16 & {$[-.71, .10]$} \\
\hline Vocab & & .03 & .03 & .12 & {$[-.03, .09]$} \\
\hline IUS & & .00 & .00 & .07 & {$[-.01, .01]$} \\
\hline Step 3 & .01 & & & & \\
\hline Constant & & -.21 & .72 & & {$[-1.63,1.22]$} \\
\hline Age & & -.01 & .01 & -.07 & {$[-.02 ., 01]$} \\
\hline Sex & & -.33 & .21 & -.17 & {$[-.74, .08]$} \\
\hline Vocab & & .03 & .03 & .13 & {$[-.02, .09]$} \\
\hline IUS & & .00 & .01 & .01 & {$[-.01, .01]$} \\
\hline SRt & & .01 & .01 & .12 & {$[-.01, .03]$} \\
\hline
\end{tabular}

Note. $n=98 . \mathrm{CI}=$ confidence interval for $B$. Vocab $=$ Mill Hill Vocabulary Scale; IUS $=$ Intolerance of Uncertainty Scale; SRt $=$ stimulus relevance for threat words. 


\section{Appendix AF}

Study 3: Hierarchical Multiple Regression with Demographics, Intolerance of Uncertainty, and Stimulus Relevance Predicting Recall of Positive Words

\begin{tabular}{|c|c|c|c|c|c|}
\hline Predictor & $\Delta R^{2}$ & $B$ & $S E B$ & $\beta$ & $95 \% \mathrm{CI}$ \\
\hline Step 1 & .00 & & & & \\
\hline Constant & & 1.42 & 1.07 & & {$[-.71,3.54]$} \\
\hline Age & & .00 & .01 & .02 & {$[-.02, .03]$} \\
\hline Sex & & .08 & .31 & .03 & {$[-.53, .69]$} \\
\hline Vocab & & -.01 & .04 & -.03 & {$[-.10, .08]$} \\
\hline Step 2 & .02 & & & & \\
\hline Constant & & 1.13 & 1.08 & & {$[-1.02,3.27]$} \\
\hline Age & & .00 & .01 & .02 & {$[-.02, .03]$} \\
\hline Sex & & .14 & .31 & .05 & {$[-.47, .76]$} \\
\hline Vocab & & -.02 & .04 & -.06 & {$[-.11, .06]$} \\
\hline IUS & & .01 & .01 & .16 & {$[-.00, .02]$} \\
\hline Step 3 & .00 & & & & \\
\hline Constant & & .95 & 1.34 & & {$[-1.70,3.61]$} \\
\hline Age & & .00 & .01 & .02 & {$[-.02, .03]$} \\
\hline Sex & & .14 & .31 & .05 & {$[-.48, .76]$} \\
\hline Vocab & & -.02 & .05 & -.05 & {$[-.11, .07]$} \\
\hline IUS & & .01 & .01 & .16 & {$[-.00, .02]$} \\
\hline SRpos & & .00 & .01 & .03 & {$[-.02, .03]$} \\
\hline
\end{tabular}

Note. $n=98 . \mathrm{CI}=$ confidence interval for $B$. Vocab $=$ Mill Hill Vocabulary Scale; IUS $=$ Intolerance of Uncertainty Scale; SRpos = stimulus relevance for positive words. 